\title{
Study of Plutonium Oxide Powder Emissions from Simulated Shipping Container Leaks
}

Prepared by J. D. Yesso, W. J. Madia, G. H. Beatty, E. W. Schmidt,

L. C. Schwendiman, J. Mishima

Battelle Columbus Laboratories

Pacific Northwest Laboratory

Operated by

Battelle Memorial Institute

Prepared for

U.S. Nuclear Regulatory

Commission 


\section{NOTICE}

This report was prepared as an account of work sponsored by an agency of the United States Government. Neither the United States Government nor any agency thereof, or any of their employees, makes any warranty, expressed or implied, or assumes any legal liability or responsibility for any third party's use, or the results of such use, of any information. apparatus product or process disclosed in this report, or represents that its use by such third party would not intringe privately owned rights.

Available from

GPO Sales Program

Division of Technical Information and Document Control

U. S. Nuclear Regulatory Commission

Washington, D. C. 20555

Printed copy price: $\$ 4.25$

and

National Technical Information Service

Springfield, Virginia 22161 
- Study of Plutonium Oxide

Powder Emissions from

Simulated Shipping

Container Leaks

Manuscript Completed: May 1980

Date Published: August 1980

Prepared by

J. D. Yesso*, W. J. Madia*, G. H. Beatty*, E. W. Schmidt*,

L C. Schwendiman**, J. Mishima**

* Battelle Columbus Laboratories

505 King Avenue

Columbus, OH 43201

** Pacific Northwest Laboratory

P.O. Box 999

Richland, WA 99352

Prepared for

Division of Safeguards, Fuel Cycle and Environmental Research

Office of Nuclear Regulatory Research

U.S. Nuclear Regulatory Commission

Washington, D.C. 20555

NRC FIN No. B2093 


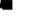

. 


\section{ABSTRACT}

To provide data to facilitate the predictions of $\mathrm{PuO}_{2}$ emissions through leaks in $\mathrm{PuO}_{2}$ shipping containers under accident conditions, a series of experiments was conducted using $\mathrm{PuO}_{2}$ powder and an experimental system designed to simulate a shipping container leak. Over two hundred experiments were completed. The experimental parameters investigated were the leak size/type, internal system pressure, agitation of the apparatus, leak orientation with respect to the powder location and the run time. No single parameter appeared to have any observable effect on the quantities of $\mathrm{PuO}_{2}$ enitted. However, there was an apparent dependency on the interaction between the orifice area and the internal pressure. The dependency took the form of a function of $A \sqrt{P}$. Although this functional form was suggested by the data, the data were not sufficient to allow a more detailed function to be determined. The results of experiments in which the run time was variable produced the observation that changes in the run time did not result in changes in the quantities of $\mathrm{PuO}_{2}$ emitted. This observation led to the conclusion that the majority of $\mathrm{PuO}_{2}$ observed is emitted during the initial pressurization of the leak tube. 


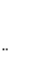


$\underline{\text { Page }}$

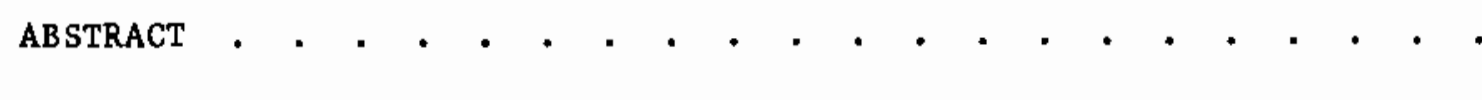

FIGURES AND TABLES . vii

INTRODUCTION .

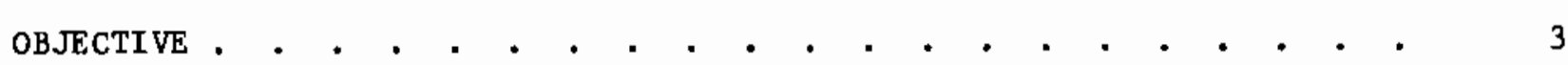

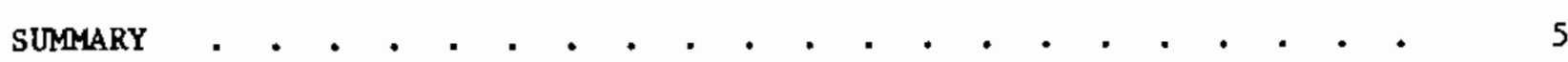

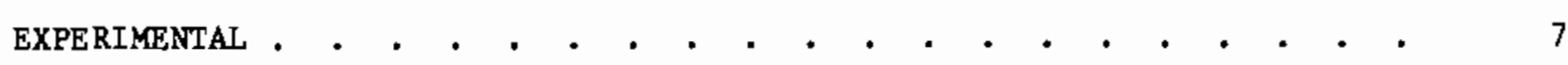

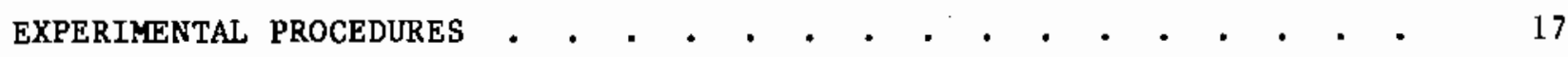

PARTICLE SIZING AND COMPARISONS . . . . . . . . . . 17

SPECIFIC ACTIVITY OF $\mathrm{PuO}_{2}$ POWDER $\quad . \quad \cdot \quad \cdot \quad \cdot \quad \cdot \quad \cdot \quad \cdot \quad 17$

HELIUM FLOW RATES . • • . • • • . • • • . . • . 21

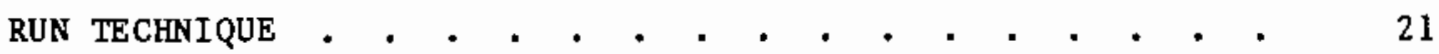

$\mathrm{ThO}_{2}$ SIMULANT EXPERIMENTS . . . . . . . . . . . . . . . . . . 31

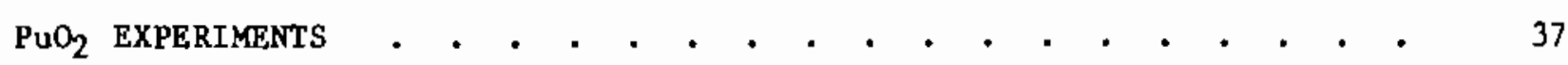

EFFECT OF EXPERIMENTAL PARAMETERS ON THE SIZE

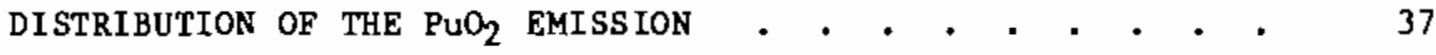

CAPILLARY LEAK EXPERIMENTS • . . . . . . • . . . . 44

EFFECT OF EXPERIMENTAL PARAMETERS ON THE QUANTITY OF

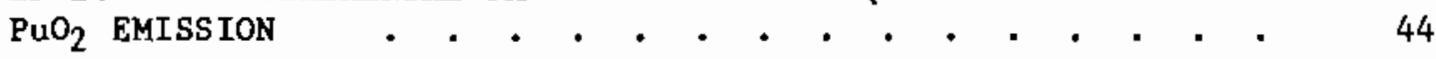

EFFECT OF EXPERIMENTAL PARAMETERS ON THE SIZE DISTRIBUTION

OF THE $\mathrm{PuO}_{2}$ EMISSION . . . . . . . . . . . . . 47

SIMULATED CRACK EXPERIMENTS . . . . . . . . . . . . 50

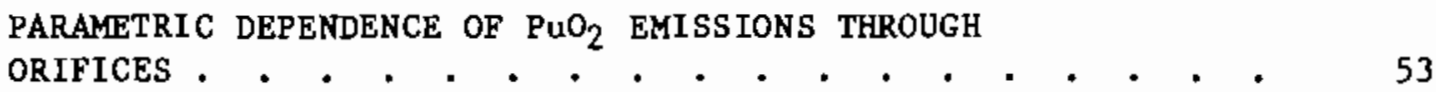

VARIABILITY OF THE DATA . . . . . . . . . . . . . . . . . 54 
TABLE OF CONTENTS

(CONT INUED)

$\underline{\text { Page }}$

BACKGROUND SUBTRACTION . • . . . . . . . . . . . 54

COLLECTION TIME DEPENDENCE • • • • • • . • • • • • • • 55

PARAMETRIC DEPENDENCY OF ORIFICE DATA.$\quad \cdot \quad \cdot \quad \cdot \quad \cdot \quad \cdot \quad \cdot \quad \cdot \quad \cdot \quad \cdot \quad \cdot \quad 56$

$\mathrm{PuO}_{2}$ MASS TO HELIUM FLOW CORREIATION

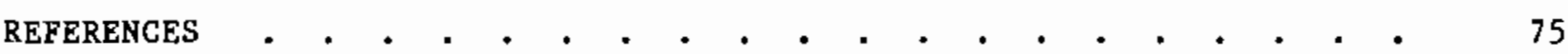

APPENDIX A - LASER OPTICAL PARTICLE MONITORING SYSTEM (LOPMS) • • . A-1

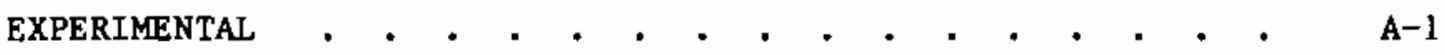

PARTICLE LEAK RATE VERSUS TIME • . . . . . . . . . A-4

PARTICLE SIZE DISTRIBUTION . . . . . . . . . . . . A A-4

CALIBRATION OF MCA . . . . . . . . . . . . . . . A-4

MEASUREMENTS WITH $\mathrm{ThO}_{2}$. . . . . . . . . . . . A-8

DISCUSSION

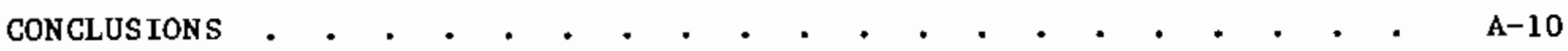

APPENDIX B - TABULATION OF LEAK RATE EXPERIMENTS DATA . • . . . - B-l 
FIGURES

$\underline{\text { Page }}$

1 SCHEMATIC DIAGRAM OF EXFERIMENTAL SET-UP USED FOR LEAK RATE

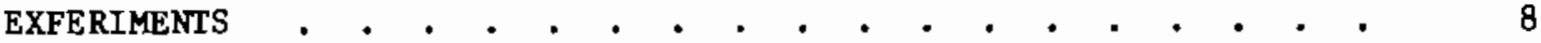

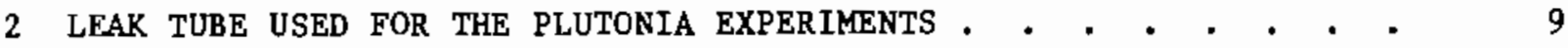

3 SCHEMATIC DRAWING SHOWING ORIFICE END OF INCONEL 600 LEAK TUBE

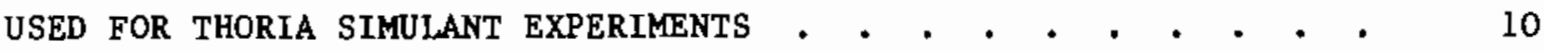

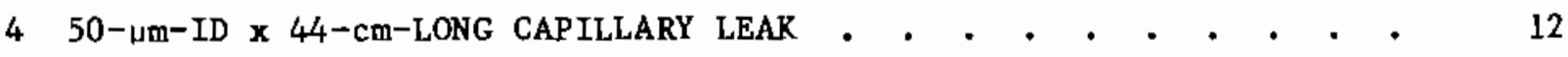

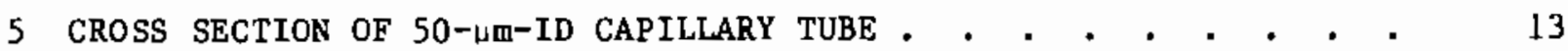

6 ATOMIZATION OF SNOOP (A LIQUID LEAR DETECTOR) BY HELIUM FLOW THROUGH THE CAPILLARY LEAK ASSEMBLY . • • . • . • . . • 15

7 SCHEMATIC SHOWING CONFIGURATION OF SIMULATED CRACK $\quad$ • . . • . 16

8 AERODYNAMIC SIZE DISTRIBUTIONS OF $\mathrm{PuO}_{2}$ and $\mathrm{ThO}_{2}$ POWDERS $\quad$ • 18

9 GEOMETRIC SIZE DISTRIBUTION OF $\mathrm{PuO}_{2}$ and $\mathrm{ThO}_{2}$ POWDER $\quad$ • $\quad$ • $\quad$. 19

10 GEOMETRIC SIZE DISTRIBUTION OF $\mathrm{PuO}_{2}$ POHDER $\quad \cdot \quad \cdot \quad \cdot \quad \cdot \quad \cdot \quad \cdot \quad \cdot \quad 20$

11 SCHEMATIC DEMONSTRATING CASCADE IMPACTOR OPERATION . • • . . 25

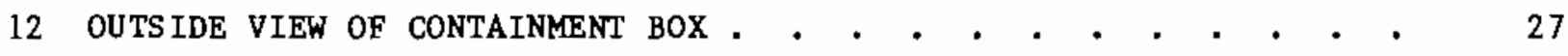

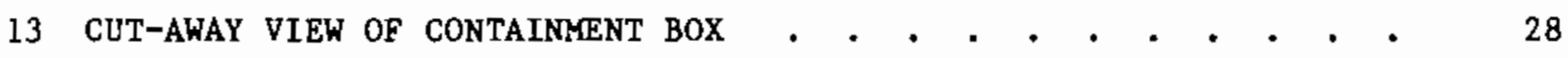

14 SCHEMATIC SHOWING EXPERIMENTAL ARRANGEMENT • • • • . • • • • . 30

15 EFFECT OF ORIFICE SIZE AND PRESSURE ON THE AVERAGE MEAN PARTICLE

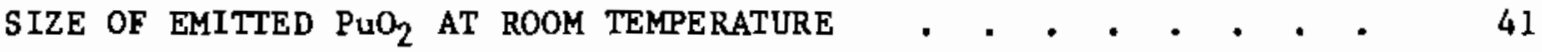

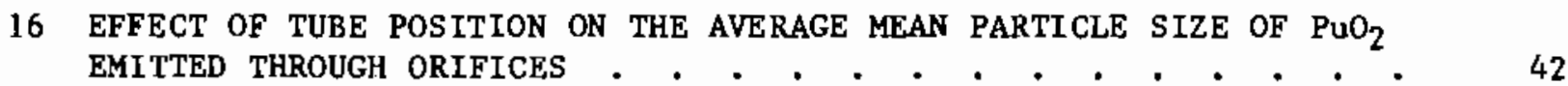

17 EFFECT OF VIBRATION ON THE EMISSION OF PuO 2 POWDER THROUGH A 20-UI ORIFICE • • • • • • • • • • • • • • • • • • • • • • • • 43

18 EFFECT OF EXPERIMENTAL CONDITIONS ON THE AVERAGE EMISSION OF PuO POWDER THROUGH A 50-LM-ID CAPILLARY AT ROOM TEMPERATJRE . . . . 46

19 PARTicle Size Distribution OF PuO 2 EMISSION THROUGH A 50- $\mu \mathrm{m}-$ ID $x$ 4.4-Cm-LONG CAPILLARY TUBE AT ROOM TEMPERATURE . . . . . . . 
FIGURES

(CONTINUED)

Page

20 DISTRIBUTION OF PLUTONIUM EMISSION (ON TRANSFORMED SCALE, Z) FOR

EXPERIMENTAL REGION I $(\mathrm{A} \sqrt{\mathrm{P}}<2000) \cdot$. . . . . . . . . . . 62

21 DISTRIBUTION OF PLUTONIUM EMISSION (ON TRANSFORMED SCALE, $z$ ) FOR

EXPERIMENTAL REGION II $(2000 \leq \mathrm{A} \sqrt{\mathrm{P}}<20,000) \cdot . \cdot . \cdot .+. \quad . \quad 66$

22 DISTRIBUTION OF PLUTONIUM EMISSION (ON TRANSFORMED SCALE, $z$ ) FOR

THREE EXPERIMENTAL REGIONS ' • • • • • • • • • • • • . 70

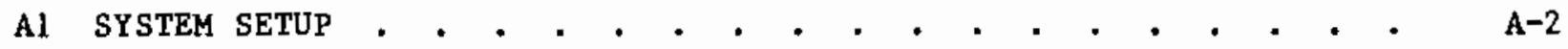

A2 LEAK TUBE USED FOR THE PLUTONIA EXPERIMENTS * . . . . . . . A-3

A3 RECORDING OSCILLOSCOPE TRACE OF PARTICLE EMISSTON RATE . . . . A-5

A4 RELATIVE COUNTS VS. CHANNEL FOR 8- $\mu$ m MONODISPERSED DOP A-6

A5 RELATIVE COUNTS VS. CHANNEL FOR ThO 2 TEST AEROSOL . . * . . A-7

\section{TABLES}

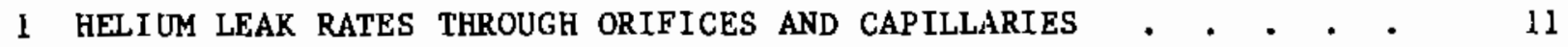

2 SPECLFIC ACTIVITY OF THE PuO 2 POWDER USED IN THE LFAK RATE EXPERIMENTS • • • • • • • • • • • • • • • • •

3 PLUTONIA ISOTOPIC COMPOSITION Pu LOT A-345 (ARHCO) ANALYZED BY

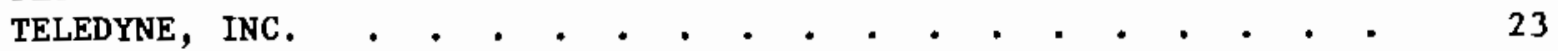

4 SUMMARY OF ROOM TEMPERATURE SIMULANT EXPERIMENTS USING ThO $\mathrm{T}_{2}$ - . 32

5 SUMMARY OF ELEVATED TEMPERATURE SIMULANT RUNS USING CASCADE

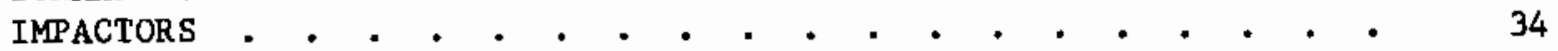

6 PARTICLE SIZE DISTRIBUTION OF PuO 2 EMITTED THROUGH A 5- $\mathrm{mm}$

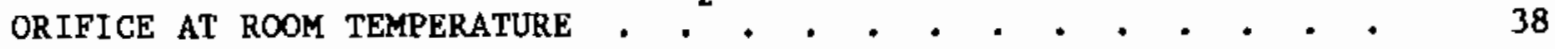

7 PARTICLE SIZE DISTRIBUTION OF PuO 2 EMITTED THROUGH A 10- $\mu \mathrm{m}$

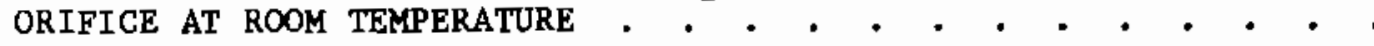


TABLES

(CONTINUED)

Page

8 PARTICLE SIZE DISTRIBUTION OF $\mathrm{PuO}_{2}$ EMITTED THROUGH A 20- $\mu \mathrm{m}$

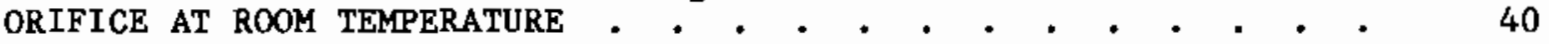

9 SUMAARY OF PuO 2 LEAK RATE EXPERIMENTS AT ROOM TEMPERATURE

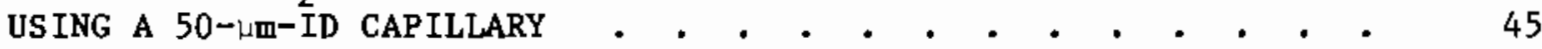

10 PARTICLE SIZE DISTRIBUTIONS OF PuO 2 EMISSION THROUGH A 50- $\mu \mathrm{m} \mathrm{x}$ 4.4-cm-LONG CAPILIARY TUBE AT ROOM TEMPERATURE . . . . . . . . . 48

11 PARAMETRIC MATRIX OF $\mathrm{PuO}_{2}$ EXPERIMENTS USING THE SIMULATED CRACK,$\cdot . \cdot \cdot \cdot \cdot \cdot \cdot \cdot \cdot \cdot \cdot \cdot \cdot \cdot \cdot \cdot \cdot \cdot \cdot \cdot \cdot 51$

12 ARITHMETIC MEAN VALUE OF $\mathrm{PuO}_{2}$ POWDER LEAKED AND THE STANDARD DEVIATION FOR EACH EXPERIMENTAL CONDITION USING THE SIMULATED CRACK CONFIGURATION (MEAN/STANDARD DEVIATION) (in NEAREST ng) . .

13 PARAMETRIC MATRIX OF PuO 2 LEAK RATE EXPERIMENTS USING

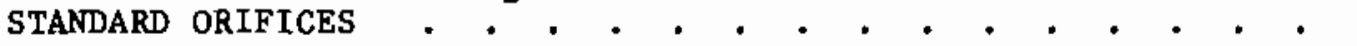

14 PARAMETRIC MATRIX OF PuO 2 LEAK RATE EXPERIMENTS USING STANDARD

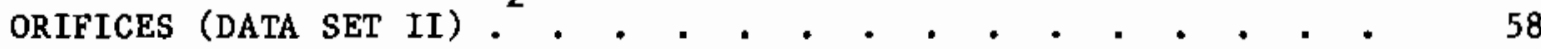

15 SUROARY STATISTICS FOR REGION I, PHASE I . • • • • . • • . 61

16 SUMMARY STATISTICS FOR REGION I, PHASE II . . . . . . . . . . 63

17 SUMOARY STATISTICS FOR REGION II, PHASE I + . • • • • • • . . 64

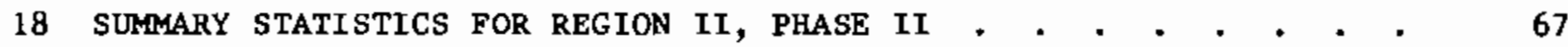

19 SUMMARY STATISTICS FOR REGION III, PHASE II • • . . . . • . 68

20 SURMARY STATISTICS FOR REGIONS I, II, AND III . • . . . . . 71

21 PLUTONIA MASS / HELIUM FLOW CORRELATION $\left(\mu \mathrm{g} / \mathrm{cm}^{3}\right)$ (PRESSURIZED LEAK TUBE) • • . • . • • . . • . • . . . • . . . 73

B1 SUMAARY OF ROOM-TEMPERATURE PUO 2 LEAR RATE EXPERIMENTS USING A

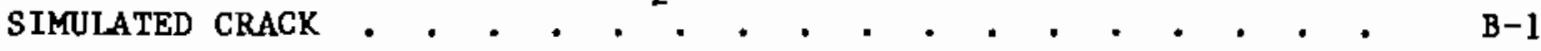

B2 SUMMARY OF ROOH-TEMPERATURE PUO 2 LEAK RATE EXPERIMENTS USING A SIMULATED CRACK; INITIAL HELIUM FLOW RATE EQUAL TO $9.8 \mathrm{cc} / \mathrm{sec}$

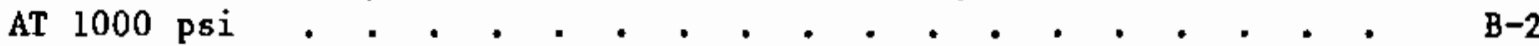


TABLES

(CONTINUED)

Page

B3 SUMMARY OF ROOM-TEMPERATURE PUO 2 LEAK RATE EXPERIMENTS USING A SIMULATED CRACK; INITIAL HELIUM FLOW RATE EQUAL TO $11.6 \mathrm{cc} / \mathrm{sec}$ AT $1000 \mathrm{pgi}$. . . . . . . . . . . . . . . . .

B4 SUMMARY OF ROOM-TEMPERATURE $\mathrm{PUO}_{2}$ LEAR RATE EXPERIMENTS USING A SIMULATED CRACK; INITIAL HELIUM FLOW RATE EQUAL TO $13.2 \mathrm{cc} / \mathrm{sec}$

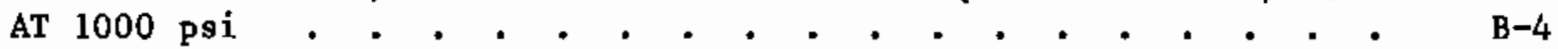

B5 SUMMARY OF ROOM-TEMPERATURE PUO 2 LEAK RATE EXPERIMENTS USING A SIMULATED CRACK; WITH AN INITIAL He FLOW RATE OF $17.3 \mathrm{cc} / \mathrm{sec}$

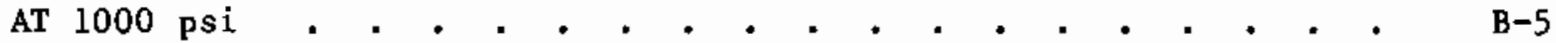

B6 SUMMARY OF PuO 2 LEAK RATE EXPERIMENTS USING A 20- m ORIFICE • • B-6

B7 SUMMARY OF PUO 2 LEAK RATE EXPERIMENTS USING A 10- m ORIFICE . . B-8

B8 SUMMARY OF PuO 2 LEAK RATE EXPERIMENTS USING A 5- m ORIFICE • • B-9

B9 SUMMARY OF $\mathrm{PUO}_{2}$ LEAK RATE EXPERIMENTS USING A 5- m ORIFICE - . B-10

B10 SUMMARY OF $\mathrm{PuO}_{2}$ LEAK RATE EXPERIMENTS USING A $10-$ m ORIFICE • • B-11

Bll SUMMARY OF $\mathrm{PuO}_{2}$ LEAK RATE EXPERIMENTS USING A 8- m ORIFICE • . B-12

B12 SUMMARY OF $\mathrm{PuO}_{2}$ LEAK RATE EXPERIMENTS USING A 20- m ORIFICE • . B-13

B13 SUMMARY OF $\mathrm{PuO}_{2}$ LEAK RATE EXPERIMENTS USING A 50- m ORIFICE * . B-15

B 14 SUMMARY OF PuO 2 LEAK RATE EXPERIMENTS USING A 50- m ORIFICE • • B-16

B 15 SUMMARY OF $\mathrm{PUO}_{2}$ LEAK RATE EXPERIMENTS USING A 50- m ORIFICE • * B-17 


\section{INTRODUCTION}

Any increased use of plutonium in power production would result in a consequent increase in the number of shipments of nuclear materials. It is imperative that the shipping containers exhibit a high degree of leak tightness in order to insure that no significant amount of plutonium is released into the environment. In general, the leak tightness of shipping containers for radioactive materials is characterized by measurements of the rate of leakage of some tracer fluid, usually a gas, from the container. $(1,2)$ The maximum permissible gas leakage rate is a function of the specific radioactive nuclides to be transported in the container. (3) It is anticipated that plutonium will be shipped in the form of a fine powder of $\mathrm{PuO}_{2}$ which is not likely to leak at a rate typical of a gas. Consequently, the application of these criteria to $\mathrm{PuO}_{2}$ shipping containers may result in unnecessarily restrictive standards for container leak tightness.

This document is a report on a Nuclear Regulatory Commission sponsored program which was designed to provide data to help establish a correlation between $\mathrm{PuO}_{2}$ particulate leakage and helium gas leakage through simulated container leaks, thus providing a data base to allow for the development of a more realistic specification for the leak tightness of $\mathrm{PuO}_{2}$ shipping containers. A primary concern is the quantity of $\mathrm{PuO}_{2}$ which might be released under conditions expected to occur as a result of a transportation accident. The original leak tightness of the container may be used to estimate the $\mathrm{PuO}_{2}$ emission.

To provide information to aid in the evaluation of the behavior of $\mathrm{PuO}_{2}$ particulate leakage, as it relates to gas leakage, under conditions to which a shipping container might be subjected, the effects of simulated container leaks were atudied. The experiments were constrained to maximize the $\mathrm{PuO}_{2}$ release. The leak types considered in this were: (1) "standard leak", consioting of a small orifice in a thin platinum disk, (2) a simulated crack made by machining a 220-microinch surface roughness in the mat-

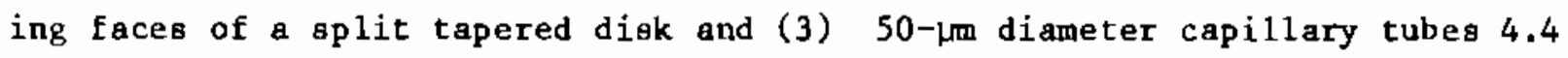
and $5.1 \mathrm{~cm}$ long. The experimental parameters invegtigated included internal gas pressure, leak orientation with respect to the powder location, agitation, run time and leak size/cype. 
As working with plutonium compounds presents certain practical handling difficulties, the approach taken was to divide the experimental effort into two distinct phases: (1) a simulant phase using Tho 2 powder, and (2) a "hot" phase using $\mathrm{PuO}_{2}$ powder. The simulant phase served to: (1) allow experimental and apparatus design to be checked out prior to transfer to the glove box necessary for the safe handling of the $\mathrm{PuO}_{2}$, (2) allow development and check out of sample collection and measurement techniques, and ( 3 ) establish a preliminary dala base.

This research was carried out by Battelle Columbus Laboratories as a part of a related services study contracted to Pacific Northwest Laboratory by the Office of Nuclear Regulatory Research. 


\section{OBJECTIVE}

The objective of this study was to provide an experimental data base that will aid in the development of techniques to assess the potential releases of plutonia powder from shipping containers under conditions expected following a transportation accident. 


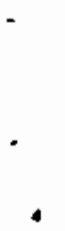




\section{SUMMARY}

A series of experiments was conducted to investigate the dependence, upon various experimental parameters, of the emission of $\mathrm{PuO}_{2}$ through small apertures. The experiments were designed around a four-parameter matrix: (1) internal gas pressure, (2) leak orientation with respect to the powder location, (3) agitation, and (4) leak size/type. For a few sets of experimental conditions the effect of the total run time was also considered. Leak types consisted of thin-plate orifices, simulated cracks consisting of split tapered disks with machined roughness on the mating

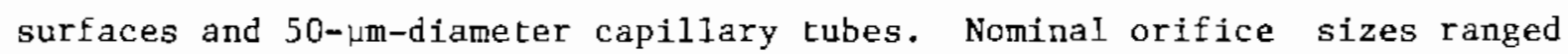
from 5- $\mu \mathrm{m}$ to 50- $\mu \mathrm{m}$ in diameter, gas pressures ranged between ambient pressure and $1250 \mathrm{psig}$ and run times ranged from "zero" to two hours. "Zero" run time refers to those experiments where the gas pressure in the experimental system was released imediately upon reaching operating pressure.

The data exhibited a high degree of irreproducibility which severely limited the ability to draw any firm conclusions concerning the parametric dependency of the data. Within the limits of the data, no apparent parametric dependence was observed for any single parameter; however, a weak dependency upon the interaction between the orifice area and the internal pressurization was observed. This is seen as an apparent functional relationship between a logarithmic transformation of the data, $z\left(z=\log _{10}(10+1000 \mathrm{x})\right.$ where $\mathrm{Z}$ is the transformed variable and $\mathrm{X}$ is the $\mathrm{PuO}_{2}$ emission in micrograms) and $\log _{10} \mathrm{~A} \sqrt{\mathrm{P}}$ where $\mathrm{A}$ is the orifice area and $\mathrm{P}$ the internal pressure. Although the relationship is suggested by the data, the data are not sufficient to allow a more exact functional dependency to be determined.

The results of the experiments where the run time was variable showed that a change in the run time did not result in a change of the quantity of $\mathrm{PuO}_{2}$ emitted. On the basis of this observation, it was concluded that the majority of the $\mathrm{PuO}_{2}$ emission observed during an experiment occurs during the initial pressurization.

Correlations between the $\mathrm{PuO}_{2}$ mass emitted during an experiments and the total helium flow produced values ranging between $3 \times 10^{-8}$ and $5 \times 10^{-3} \mathrm{Hg}$ of $\mathrm{PuO}_{2} / \mathrm{cm}^{3}$ of helium under internal pressurizaticin of 500 to 1250 psig. 


\section{EXPERIMENTAL}

The experimental setup used for both phases of the program is shown schematically in Figure 1. The essential components of the system are the leak tube, used to contain the $\mathrm{ThO}_{2}$ or $\mathrm{PuO}_{2}$ powder and support the leak assembly, a helium supply with pressure regulation, and a samping device. The leak tube itself is constructed of Inconel Alloy 100, has an overal1 length of $12.7 \mathrm{~cm}$ and an ID of $0.64 \mathrm{~cm}$. The reservoir volume is approximately $4 \mathrm{cc}$. Helium, to provide the necessary internal pressurization, is supplied through a $0.64-c m$ tube located at midlength. The leak tube used for the $\mathrm{PuO}_{2}$ experiments is shown in Figure 2. The tube used for the simulant experiments was similar in design, but had the cap shown in rigure 3.

The leak assemblies were mounted in the end of the leak tube and sealed with stainless stęl gaskets. The orifices used were prepared by the laser drilling of holes ranging between 5 and $50 \mu \mathrm{m}$ in $0.10-\mathrm{mm}$ platinum disks $9.5-\mathrm{mm}$ in diameter. The orifices were examined microscopica1ly prior to use to insure that the diameters were correct and the openings unobstructed. The flow rates of helium through 5-, 10-, 20- and 50-um diameter orifices and a 50- $\mu \mathrm{m}$ ID capillary tube were measured using either the bubble-tube or pressure-drop method or both. The results of these measurements are presented in Table 1. These data apply to clean orifices installed in an empty leak tube; the actual leaks observed during the particle emission experiments vary from these somewhat.

Capillary leak assemblies were prepared by silver brazing type 304 stainless steel capillary tubing having an ID of $50 \mu \mathrm{m}$ and an OD of $125 \mu \mathrm{m}$ into a type 304 stainless steel support tube having an ID of $175 \mu \mathrm{m}$ and OD of $1575 \mathrm{~mm}$. This tubing assembly was brazed to a type 304 stainless stee 1 disk sized to fit into the end of the leak tube. Assemblies of length 4.4 and $5.1 \mathrm{~cm}$ were prepared. The $4.4-\mathrm{cm}-1$ ong assembly is shown in Figure 4 . As might be expected, the inner surfaces of the capillary exhibit appreciable irregularity. Figure 5, a photomicrograph of a capillary cross section, clearly illustrates this irregularity. 


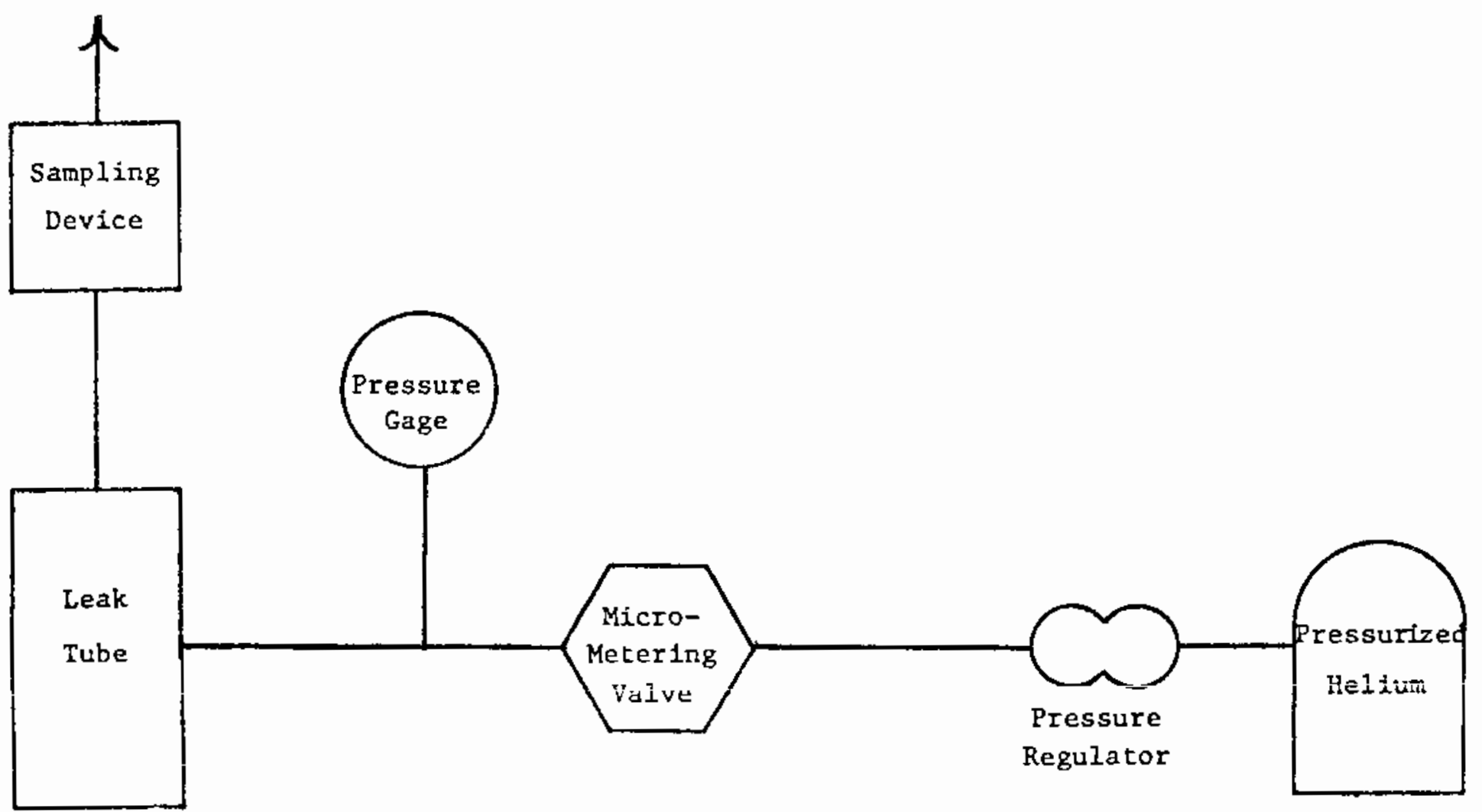

FIGURE 1. SCHEMATIC DIACRAM OF EXPERIMENTAI. SET-UP USED FOR LEAK RATE EXPERIMENTS 


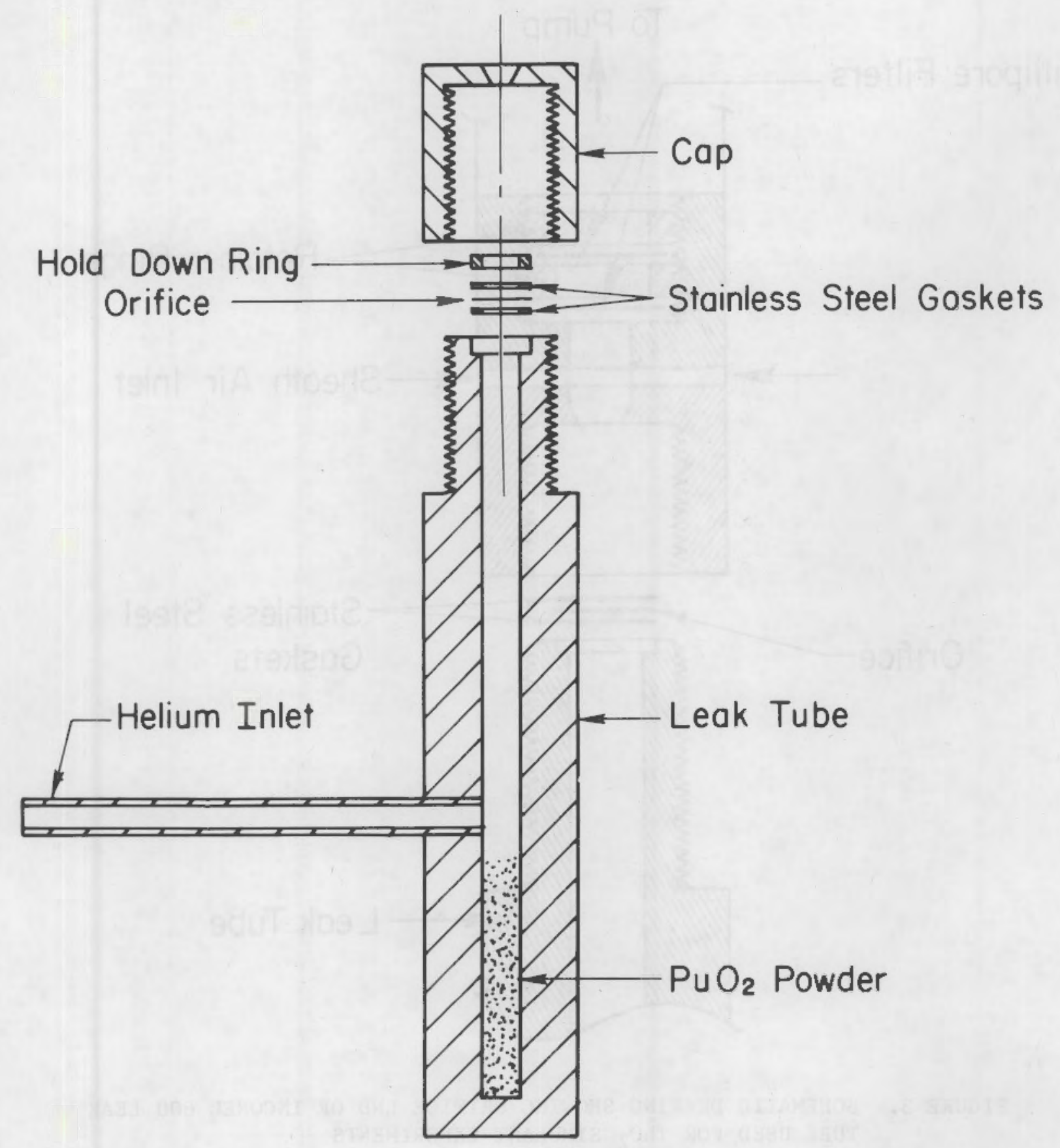

FIGURE 2. LEAK TUBE USED FOR THE $\mathrm{PuO}_{2}$ EXPERIMENTS 

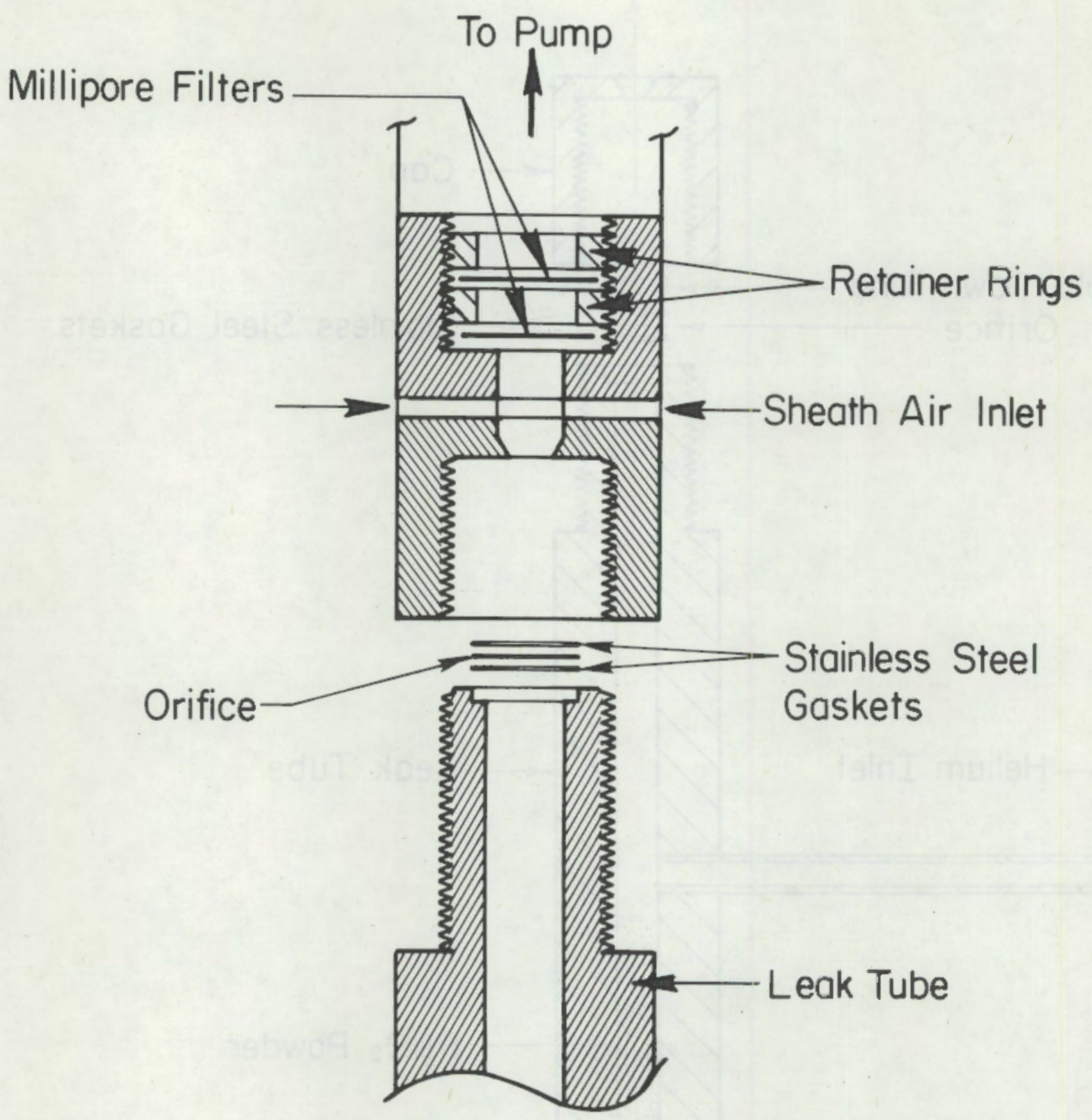

FIGURE 3. SCHEMATIC DRAWING SHOWING ORIFICE END OF INCONEL 600 LEAK TUBE USED FOR ThO ${ }_{2}$ SIMULANT EXPERIMENTS 
TABLE 1. HELIUM LEAK RATES THROUGH ORIFICES AND CAPILLARIES

\begin{tabular}{|c|c|c|}
\hline Type of Leak & $\begin{array}{c}\text { Pressure, } \\
\text { psig }\end{array}$ & $\begin{array}{l}\text { Helium } \\
\text { Leak Rate, } \\
\text { std } \mathrm{cc} / \mathrm{sec}\end{array}$ \\
\hline 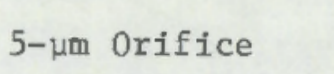 & 0 & $1.25 \times 10^{-5}$ \\
\hline$"$ & 500 & 0.39 \\
\hline$"$ & 1000 & 0.77 \\
\hline 10-um Orifice & ; & $5.03 \times 10^{-5}$ \\
\hline$"$ & 500 & 1.39 \\
\hline$"$ & 1000 & 2.89 \\
\hline $20-\mu \mathrm{m}$ Orifice & 0 & $2.02 \times 10^{-4}$ \\
\hline$"$ & 500 & 4.21 \\
\hline$"$ & 1000 & 8.09 \\
\hline 50- $\mu \mathrm{m}$ Orifice & 0 & $1.25 \times 10^{-3}$ \\
\hline$"$ & 500 & - \\
\hline$"$ & 1000 & 58.0 \\
\hline 50-um Capillary & 0 & $2.86 \times 10^{-6}$ \\
\hline$"$ & 500 & 0.3 \\
\hline$"$ & 1000 & 0.8 \\
\hline
\end{tabular}




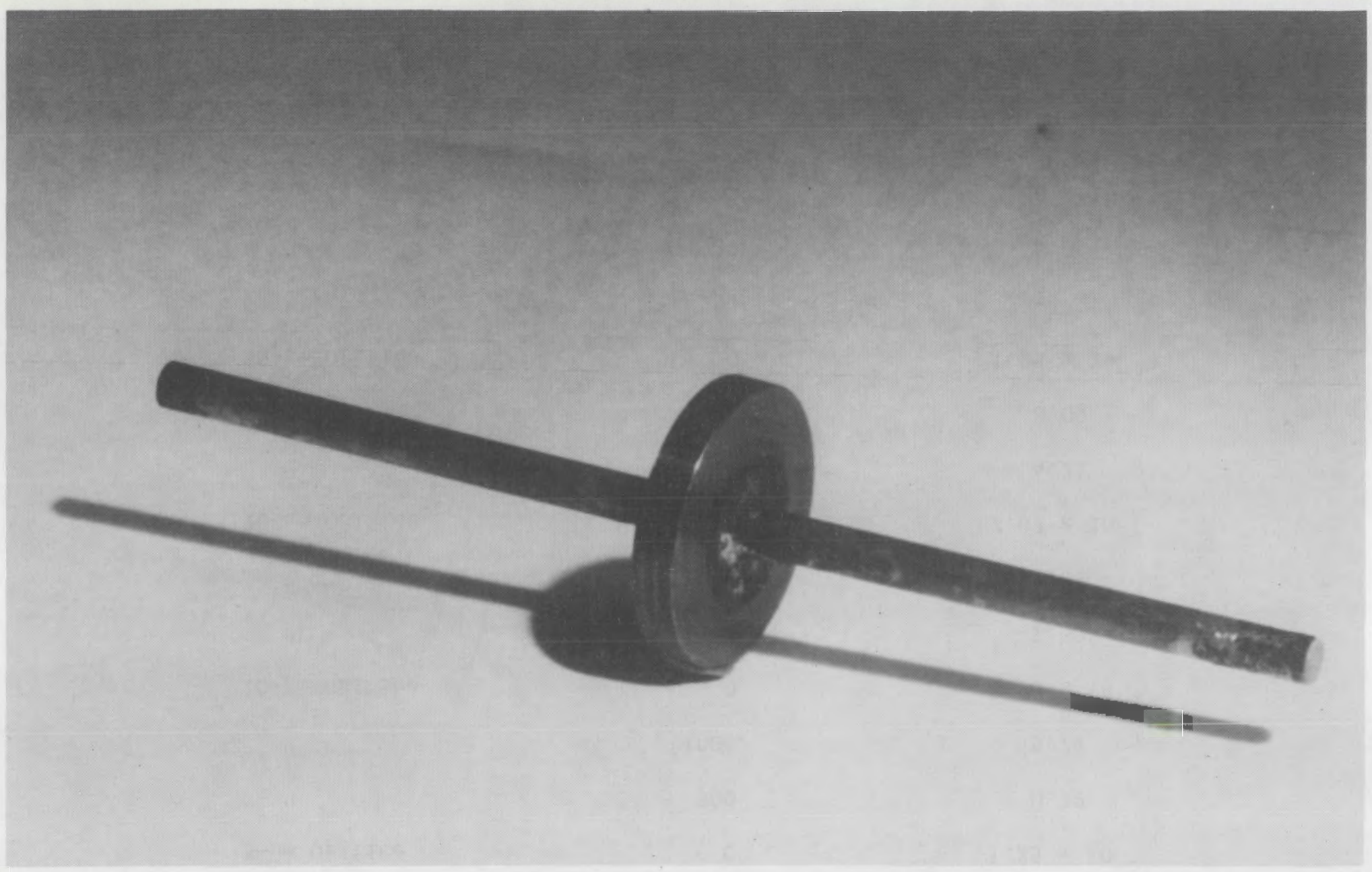

FIGURE 4. 50- $\mu \mathrm{m}$-ID $\times 4.4-\mathrm{cm}$-LONG CAPILLARY LEAK 


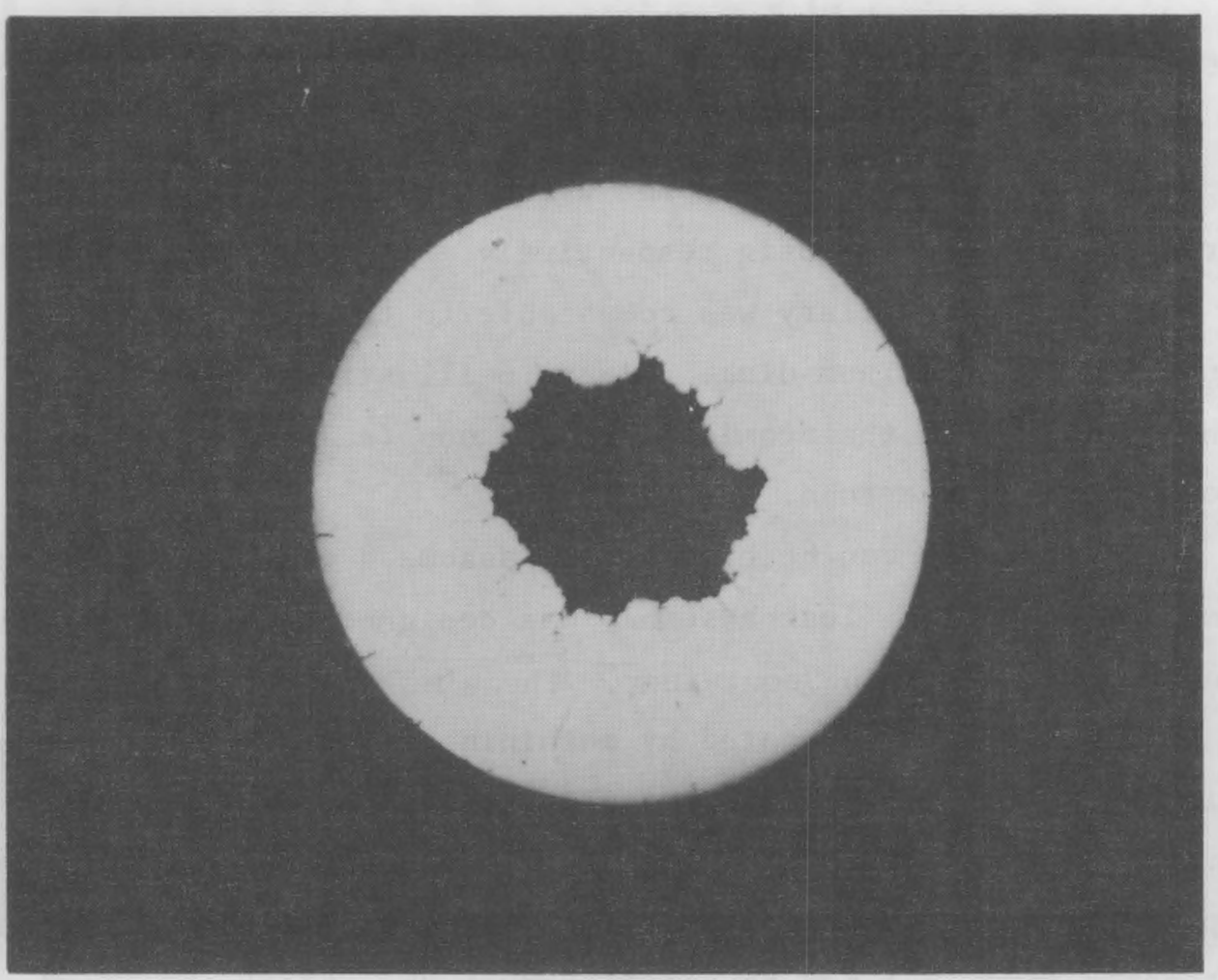

FIGURE 5. CROSS SECTION OF 50- $\mu \mathrm{m}$-ID CAPILLARY TUBE 
The helium flow rate through the $4.4-\mathrm{cm}-1$ ong capillary leak assembly. was measured to be 0.82 and $0.30 \mathrm{cc} / \mathrm{sec}$ using the pressure decay method at pressures of 1000 and 500 psig respectively. At 1000 psig, the helium flow rate through the capillary was comparable to that observed for a $5-\mu m$ orifice in a $0.10-\mathrm{mm}$ platinum disk. Figure 6 illustrates the flow of helium through the capillary by the atomization of Snoop (a liquid leak detector) applied to the end of the tube.

As a leak path can be expected to assume a wide varlety of configurations, an additional leak assembly was designed to simulate a crack In a plutonia powder shipping container. The simulated crack, shown schematically in Figure 7, was prepared by machining a surface roughness of 220 microinches on the mating faces of a split tapered disk. During machining, the disk was positioned such that the grooves would interlock when the disk pieces were placed together. The disk was designed to fit into a matching tapered ring that may be positfoned at the end of the standard leak tube. The crack spacing, and consequently the helium leak rate, was adjusted by varying the amount of torque applied to the leak tube retaining nut. The observed leak rates varied between 6.1 and $17.3 \mathrm{cc} / \mathrm{sec}$ at $1000 \mathrm{psig}$ and various applied torques.

Two types of sampling devices were used for the $\mathrm{ThO}_{2}$ simulant experiments. Most of the experiments used the arrangement shown in Figure 2 where Millipore ${ }^{(a)}$ filters are mounted in the leak tube cap. The other sampling device which was also used for some of the $\mathrm{PuO}_{2}$ experiments was a cascade impactor. This is a device which employs inertial deposition to collect aerosol particles and classify them according to size.

(a) Manufactured by Millipore Corp., Bedford, MA 


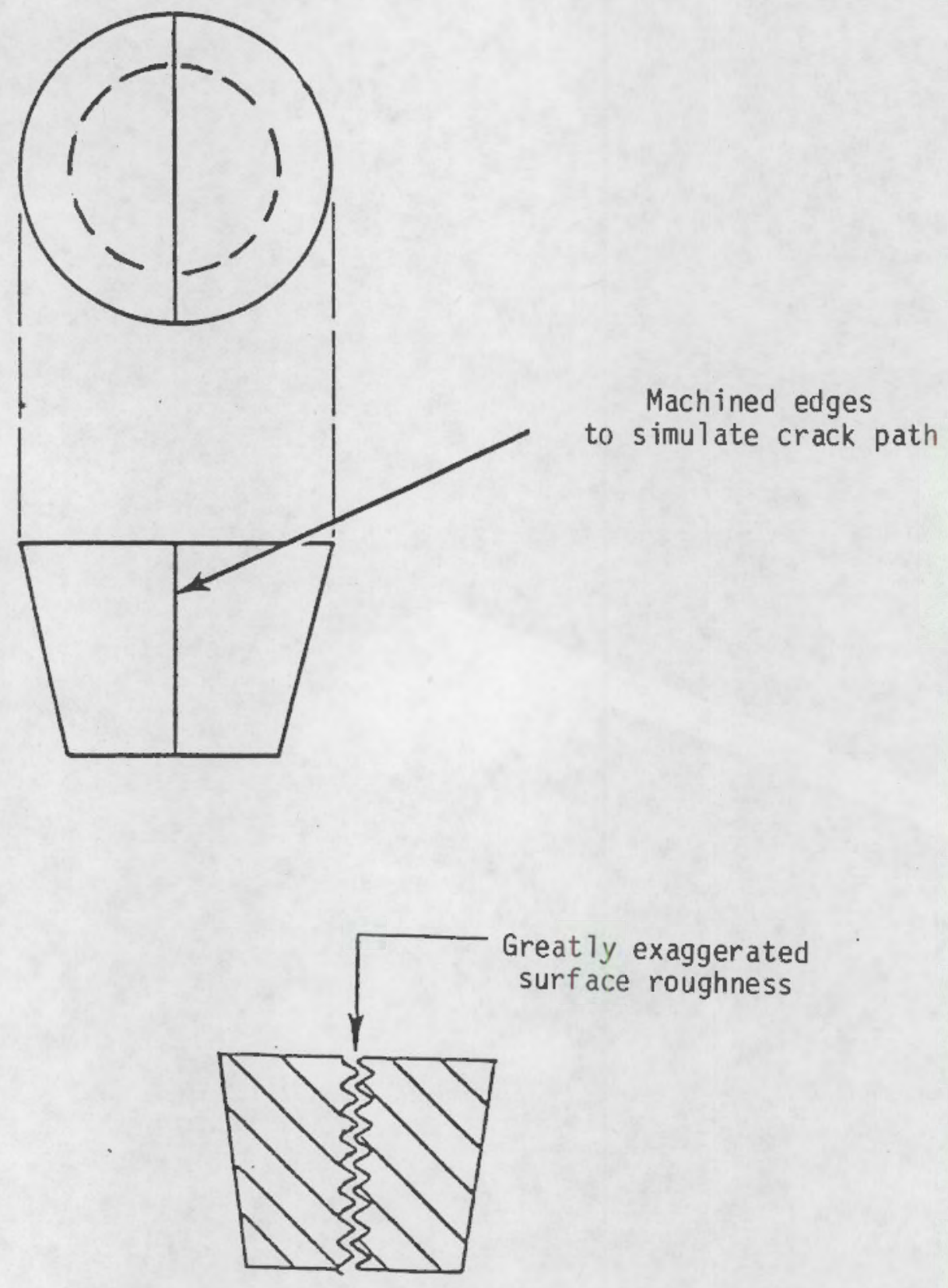

FIGURE 7. SCHEMATIC SHOWING CONFIGURATION OF SIMULATED CRACK 


\section{EXPERIMENTAL PROCEDURES}

\section{Particle Sizing and Comparisons}

Prior to its final selection as the simulant material the $\mathrm{ThO}_{2}$ particle size distribution was compared with that of the $\mathrm{PuO}_{\text {, }}$ powder to ensure that the $\mathrm{ThO}_{2}$ would be an appropriate simulant. The $\mathrm{ThO}_{2}$ was first screened using the 325 -mesh $(44-\mu \mathrm{m})$ sieve to remove any large particles and then sized using the Battelle cascade impactor. Figure 8 shows the results of these measurements, as well as the nominal size distribution as obtained from Schwendiman ${ }^{(4)}$, of the $\mathrm{PuO}_{2}$ powder for which the $\mathrm{ThO}_{2}$ serves as a simu1ant. The agreement, though not exact, was adequate for this study with the mean diameters being 7.8- $\mu \mathrm{m}$ and $8.3-\mu \mathrm{m}$ for $\mathrm{ThO}_{2}$ and $\mathrm{PuO}_{2}$ respectively.

Figure 8 presents aerodynamic size distributions for the powders. By applying a density correction, the geometric size distribution for the $\mathrm{ThO}_{2}$ powder may be determined. Figure 9 shows the resulting $\mathrm{ThO}_{2}$ distribution and the $\mathrm{PuO}_{2}$ distribution obtained from the vendor ${ }^{(a)}$ in May 1974. Again, although not precise, the $\mathrm{ThO}_{2}$ appears to be a representative $\mathrm{PuO}_{2}$ simulant for use in the cold-bench experiments.

The $\mathrm{PuO}_{2}$ particle size distribution was later determined by the cascade impactor technique and compared with the vendor data. $\mathrm{PuO}_{2}$ powder was aspirated froma reservoir containing $0.87 \mathrm{~g}$ of $\mathrm{PuO}_{2}$ into a plexiglas chamber to which a cascade impactor was attached. The vendor distribution was obtained using the Coulter counter method. Figure 10 compares the two distributions. As is evident from the curves, there is exceptionally good agreement between the distributions determined by the two different techniques. Both sets of data indicate mean particle size of $2.5 \mu \mathrm{m}$.

\section{Specific Activity of $\mathrm{PuO}_{2}$ Powder}

As only minute quantities of $\mathrm{PuO}_{2}$ are expected to be emitted from the leak assemblies during the experiments, gravimetric analysis was felt to be impractical and chemical analysis too costly and time consuming. Con-

(a) Plutonium dioxide powder obtained from ARHCO (Alantic Richfield Hanford Co.), Richland, WA. 


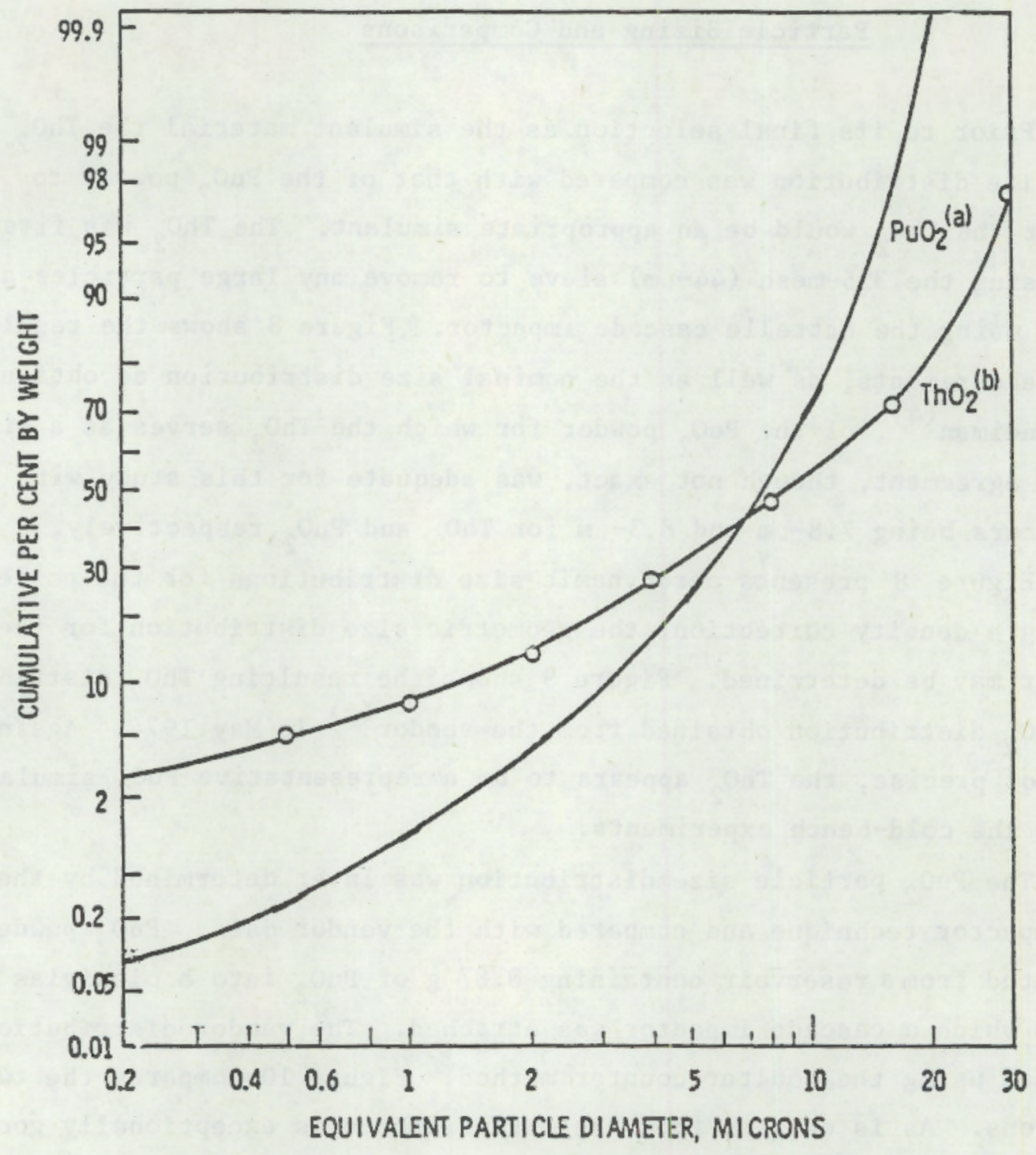

(a) Nominal $\mathrm{PuO}_{2}$ size distribution (Schwendiman 1977)

(b) Nominal $\mathrm{ThO}_{2}$ size distribution (determined by L. Miga of Battelle Columbus Laboratories, December 18, 1976)

FIGURE 8. AERODYNAMIC SIZE DISTRIBUTIONS OF PuO 2 AND ThO 2 POWDERS 


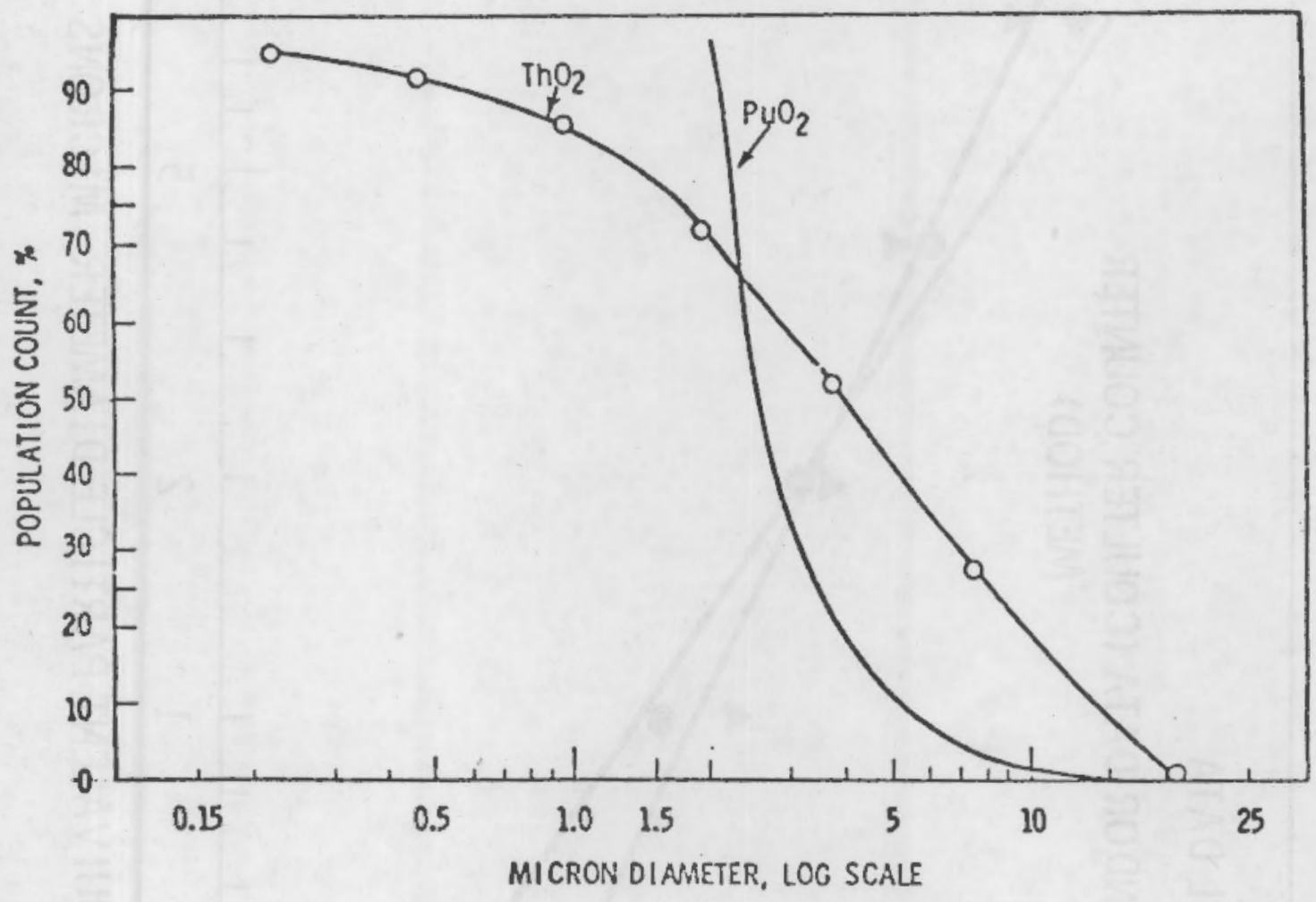

FIGURE 9. GEOMETRIC SIZE DISTRIBUTION OF $\mathrm{PuO}_{2}$ AND $\mathrm{ThO}_{2}$ POWDER 


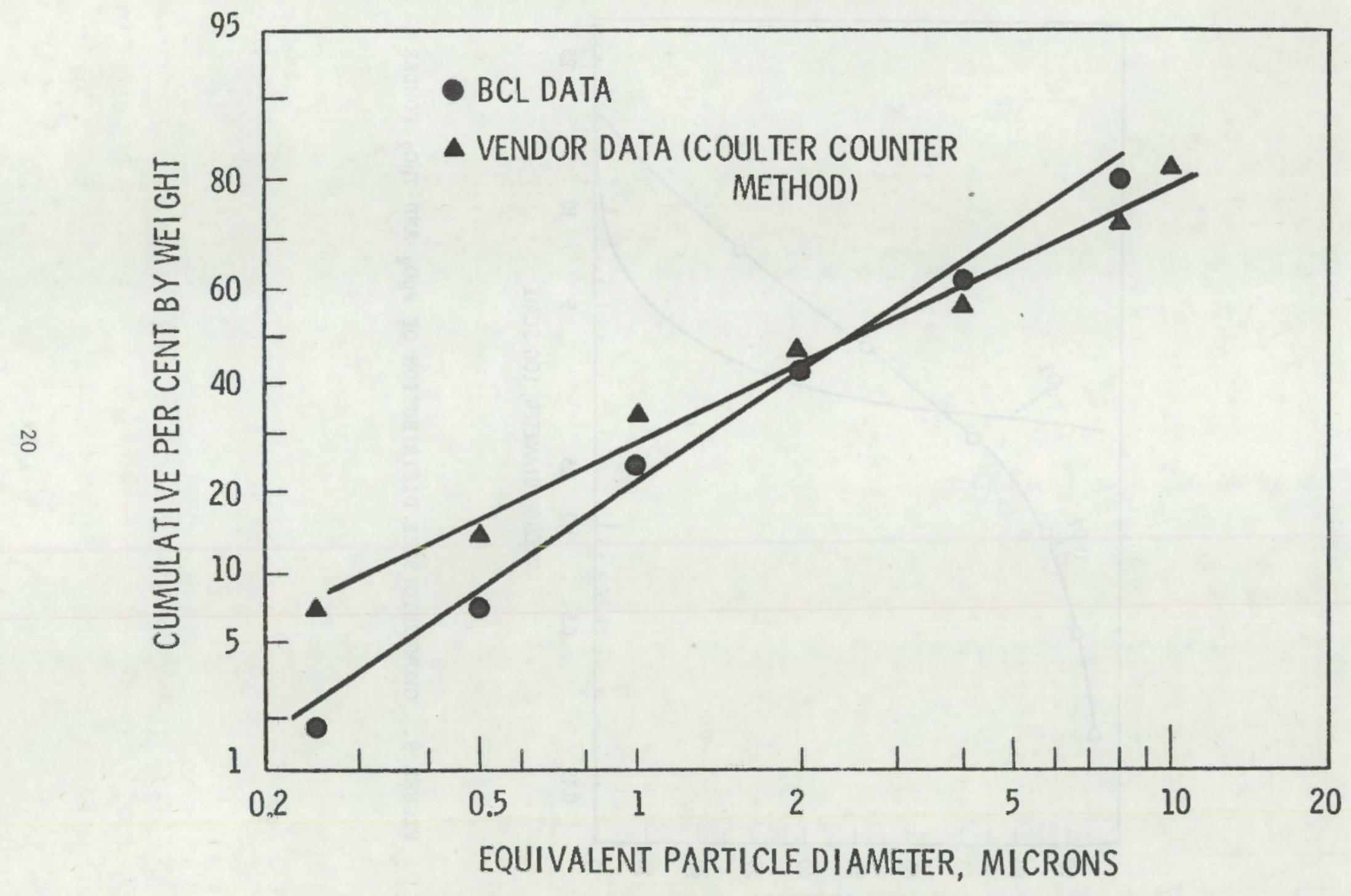

FIGURE 10. GEOMETRIC SIZE DISTRIBUTION OF $\mathrm{PuO}_{2}$ POWDER 
sequently, radioassay analysis was selected as the technique used to determine the quantities of $\mathrm{PuO}_{2}$ collected.

The radioassay technique chosen employed the counting of the alpha particles emitted by the plutonium. This technique necessitates the correction of counting data for background, detection efficiency and the specific activity of the $\mathrm{PuO}_{2}$.

In order to obtain an actual specific activity of the $\mathrm{PuO}_{2}$ powder being used in this study, three pairs of plutonium standards were prepared by dissolving the powder and depositing different quantities of the solution on small watch glasses. The solvent was evaporated by heating the watch glasses on a hot plate. The activity of these standards was measured and the specific activity was determined. The results are tabulated in Table 2 . The isotopic composition of the powder as determined by Teledyne, Inc., is presented in Table 3. A specific activity calculated from these values is also shown in the table. As is apparent, the calculated value of $0.098 \mathrm{Ci} / \mathrm{g}$ is in excellent agreement with the measured activity of $0.096 \pm 0.004 \mathrm{Ci} / \mathrm{g}$.

\section{Helium Flow Rates}

Specifications for acceptable container leak sizes may be stated in terms of the quantity of helium leaked with respect to time. In order to provide any correlative information concerning particle emissions and gas flow, the flow rates must be adequately determined. Helium flow rates through the leak assemblies were determined by using either a bubble flow tube or calculations based on the system volume and the rate of decay of a closed (except for the leak assembly) system. This latter method is the proposed ANSI standard leak test on packages for shipment of radioactive materials, N-14.5 (12th draft). (1) For those runs conducted at ambient internal pressures, the approximate leak rate was calculated assuming molecular diffusion of helium into air.

\section{$\underline{\text { Run Technique }}$}

All experiments, unless noted otherwise, were conducted with a nominal collection time of ten min. Notes collection times refer to the time during which a given set of experiments conditions are extant. 
TABLE 2. SPECIFIC ACTIVITY OF THE PUO POWDER USED IN THE

LEAR RATE EXPERIMENTS

\begin{tabular}{cc}
\hline Standard Number & Specific Activicy, Ci/g \\
\hline $1 \mathrm{~A}$ & 0.09 \\
$1 \mathrm{~B}$ & 0.092 \\
$2 \mathrm{~A}$ & 0.099 \\
$2 \mathrm{~B}$ & 0.105 \\
$5 \mathrm{~A}$ & 0.095 \\
$5 \mathrm{~B}$ & $\underline{0.095}$ \\
& Average $=0.096 \pm 0.004$ a.d. \\
\hline
\end{tabular}

As indicated above, the average specific activity of the standards is $0.096 \pm 0.004 \mathrm{Ci} / \mathrm{g}$. 
TABLE 3. PLUTONIA ISOTOPIC COMPOSITION

Pu LOT A-345 (ARHCO)

ANALYZED BY TELEDYNE, INC.

\begin{tabular}{lc}
\hline Isotope & Weight Percent \\
Pu-238 & 0.108 \\
-239 & 86.881 \\
-240 & 11.359 \\
-241 & 1.432 \\
-242 & 0.220 \\
\hline
\end{tabular}

Specific Activity: $0.098 \mathrm{Ci} / \mathrm{g}$ (Calculated)

$0.096 \pm 0.004 \mathrm{Ci} / \mathrm{g}$ (Measured) 
For all runs the leak tube was agitated for 1 min to disperse the powder and the sample collection device was operated for 1 min prior to pressurization and 1 min following depressurization in order insure that collection takes place during the entire period of particle flow.

At the mid-point of each run, the helium flow rate was determined using the pressure decay method.

The so-called "zero-time" runs were conducted such that the leak tube was pressurized for the minimum amount of time that was practical. This was accomplished by allowing the internal pressure to reach the desired value and then immediately isolating the leak tube from the high pressure line and releasing the helium through a bleed valve. The entire operation required approximely $1 \mathrm{~min}$.

The impactor operates on the principle that particles moving in an airstream will strike a slide paced in their path if they possess sufficient inertia to overcome the drag exerted by the airstream which will pass around the slide. Figure 11 illustrates the operation of the impactor. As the aerosol approaches the slide after passing through the nozzle, the larger particles will impact on the slide while the smaller particles are carried along with the airstream. Because each jet is smaller than the preceding one, the velocity of the airstream will increase with each successive stage of the impactor and complete size classification is achieved. Those particles too small to be collected in the fifth and final stage are collected on a high-efficiency membrane filter.

When size classification was not desired for the plutonium experiments, the impactor was replaced with a device similar in configuration, but containing a membrane filter only to provide complete particle collection.

When desired, agitation may be applied to the leak tube through a mechanical vibrator ${ }^{(a)}$ directly coupled to the leak tube. The purpose of the vibration, when used, was to maximize the aerosolization of the powder during experiments.

(a) Scripto Vibratool ${ }^{\circ}, 120 \mathrm{cps}, 1 / 32$-in displacement, uncoupled. 


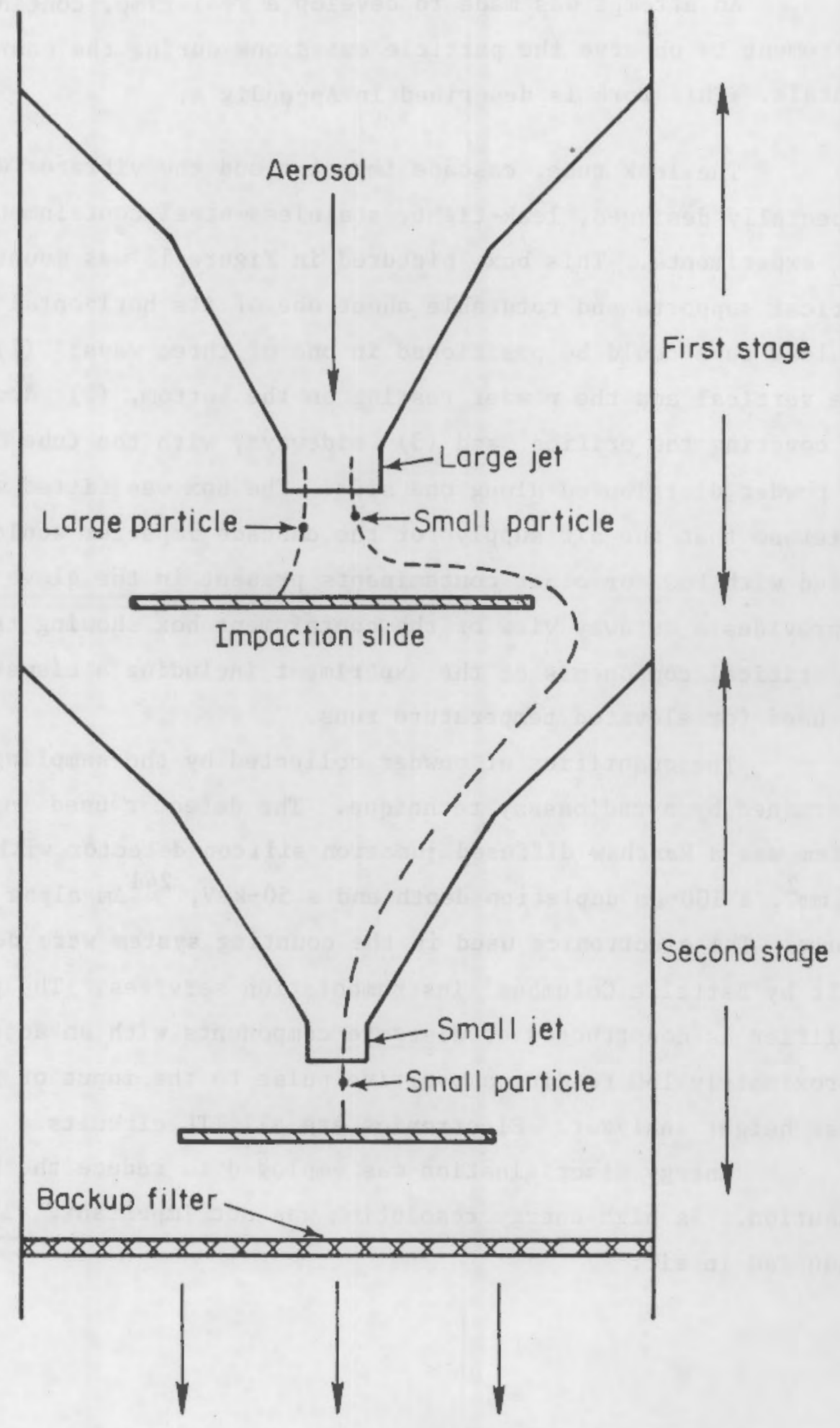

FIGURE 11. SCHEMATIC DEMONSTRATING CASCADE IMPACTOR OPERATION 
An attempt was made to develop a real-time, continuous monitoring instrument to observe the particle emissions during the course of the experimentals. This work is described in Appendix A.

The leak tube, cascade impactor and the vibrator were mounted in a specially designed, leak-t1ght, stainless-steel containment box for the $\mathrm{PuO}_{2}$ experiments. This box, pictured in Figure 12 was mounted on two vertical supports and rotatable about one of its horizontal axes such that the leak tube could be positioned in one of three ways: (1) up, with the tube vertical and the powder resting on the bottom, (2) down, with the powder covering the orifice, and (3) sideways, with the tube horizontal and the powder distributed along one side. The box was fitted with an absolute filter so that the air supply for the cascade impactor would not be contam- " inated with $\mathrm{PuO}_{2}$ or other contaminants present in the glove box. Figure 13 provides a cutaway view of the containment box showing the piacement of the critical components of the experiment including a clamshell-type furnace used for elevated temperature runs.

The quantities of powder collected by the sampling device were determined by a radioassay technique. The detector used in the counting system was a Harshaw diffused junction silicon detector with a surface area of $450 \mathrm{~mm}^{2}$, a $100-\mu m$ depletion depth and a 50-keV, ${ }^{241}$ Am alpha resolution in vacuum. The electronics used in the counting system were designed and built by Battelle Columbus' instrumentation services. The preamplifieramplifier is constructed of discrete components with an adjustable gain of approximately 150 feeding a positive pulse to the input of the single channel pulse height analyzer. Electronics are all TTL circults.

Energy discrimination was employed to reduce the background contribution. As high-energy resolution was not important, all counting was conducted in air. 



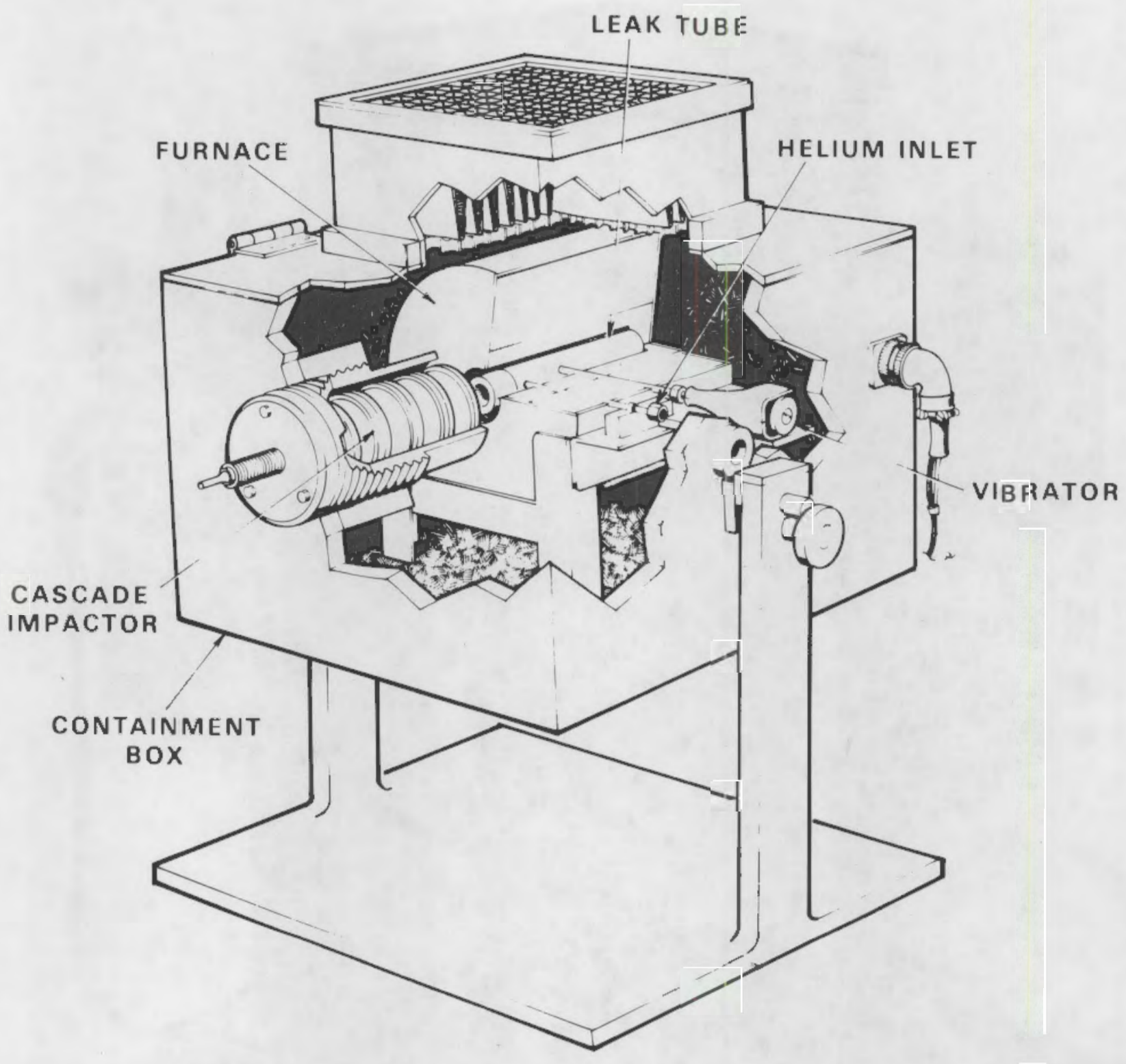

FIGURE 13. CUT-AWAY VIEW OF CONTAINMENT BOX 
The entire experimental system was housed in a stainless steel glove box ${ }^{(\mathrm{a})}$ for the experiments using $\mathrm{PuO}_{2}$ powder. Prior to use, the glove box was pressurized with helium and leak tested with a helium mass spectrometer type leak detector. No detectable leakage was observed with the leak detector set on the $1 \times 10^{-8}$ torr range. Figure 14 shows, schematically, the arrangement of the experimental system in the glove box. To a1low monitoring of the helium flow, an "Omniflow" turbine flow-meter (b) was installed in the experimental system. The "Omniflow" is an in-line flow metering device which provides digital flow information. The flow rates obtained are in terms of the actual pressures, they must be converted to standard flow rates. The flow-meter was calibrated in the experimental system using the pressure decay method to insure that flow rate information could be related to previous runs. Thirty data points were used in the calibration and were fitted to a straight line with a linear correlation coefficient of 0.995 . The calibration curve reveals that the minimum flow which can be reliably measured by the flow-meter is approximately 0.1 acc/sec.

(a) Obtained from Stainless Engineering Company, Boulder, co

(b) Obtained from Flow Technology, Inc., P.0. Box 21346, Phoenix, AR 


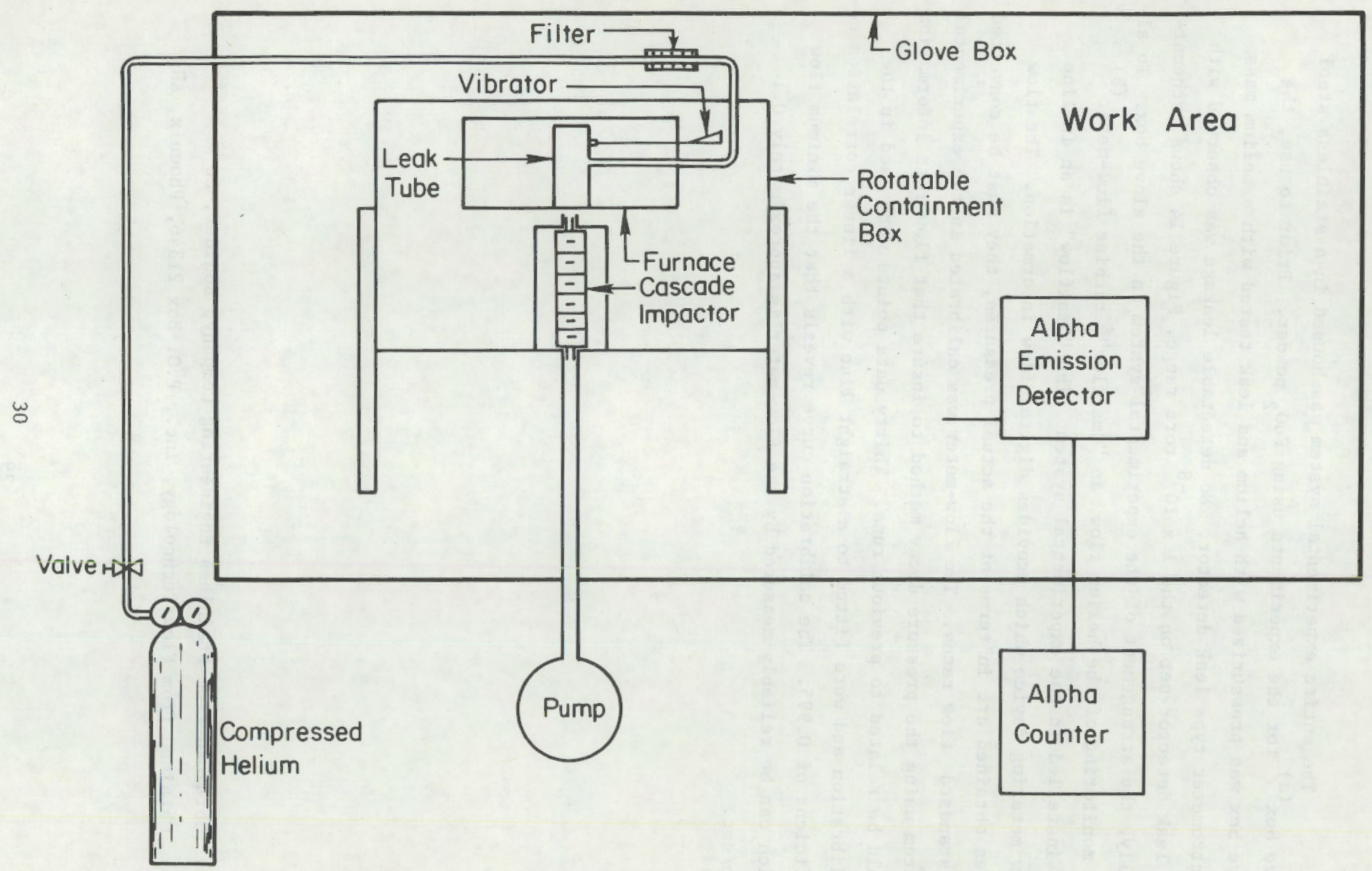

FIGURE 14. SCHEMATIC SHOWING EXPERIMENTAL ARRANGEMENT 


\section{$\mathrm{ThO}_{2}$ SIMULANT EXPERIMENTS}

Preliminary bench-top experiments were conducted using $\mathrm{ThO}_{2}$ powder as a simulant for the $\mathrm{PuO}_{2}$ powder. The objective of these experiments was to allow the check-out and development of the experimental system and technique prior to initiation of the $\mathrm{PuO}_{2}$ experiments and to establish a preliminary data base concerning the effects of orifice size, leak-tube orientation, internal system pressure and agitation on the emission of powders through the leak assemblies.

The simulant experiments were conducted at nominal pressures of 500 and $1000 \mathrm{psi}$, temperatures of ambient, $400^{\circ} \mathrm{C}$ and $815^{\circ} \mathrm{C}$ using orifices of $5,10,20$ and $50-\mu m$ diameters. The $\mathrm{ThO}_{2}$ emissions were collected on membrane filters mounted in the leak tube cap for the ambient temperature runs. The cascade impactor was used during the elevated temperature runs. The exact conditions for each experiment, along with the results, are presented in Tables 4 and 5 . The quantities of $\mathrm{ThO}_{2}$ collected were determined using either an alpha radioassay technique or X-ray fluorescence.

On the basis of this rather limited set of data, the following observations have been made:

(1) With a few exceptions, no $\mathrm{ThO}_{2}$ release was noted for orifices less than $50 \mu \mathrm{m}$ in diameter under any conditions. This does not mean that no release occurred, but that it was not readily detectable with the $\mathrm{ThO}_{2}$ simulant.

(2) At room temperature, more $\mathrm{ThO}_{2}$ was emitted through a 50- $\mu \mathrm{m}$ orifice with the orifice in the down position and without vibrating the leak tube as compared to a similar experiment with vibration (see Th 14 and Th 17).

(3) Based on the results of Runs $17,17 \mathrm{a}, 17 \mathrm{~b}$, and $17 \mathrm{c}$, the emission of $\mathrm{ThO}_{2}$ through a $50-\mu \mathrm{m}$ orifice at toom temperature was reproducible within a factor of about 2 for the leak-down position.

(4) Results with respect to position and vibration are rather ambiguous. For example, considerable $\mathrm{ThO}_{2}$ 
TABLE 4. SUMMARY OF ROOM TEMPERATURE SIMULANT EXPERIMENTS USING $\mathrm{ThO}_{2}$

\begin{tabular}{|c|c|c|c|c|c|c|c|c|c|c|c|c|}
\hline \multirow[b]{2}{*}{$\begin{array}{l}\text { Run } \\
\text { No. }\end{array}$} & \multirow{2}{*}{$\begin{array}{c}\text { Orifice } \\
\text { Diameter, } \\
\mu m\end{array}$} & \multirow[b]{2}{*}{$\begin{array}{c}\text { Tube } \\
\text { Position }\end{array}$} & \multirow{2}{*}{$\begin{array}{l}\text { Nominal } \\
\text { Helium } \\
\text { Pressure } \\
\text { psig }\end{array}$} & \multirow{2}{*}{$\begin{array}{c}\text { Tempera- } \\
\text { ture } \\
\text { C }\end{array}$} & \multirow{2}{*}{$\begin{array}{c}\text { Length } \\
\text { of Run, } \\
\text { hr }\end{array}$} & \multirow[b]{2}{*}{ Vibration } & \multirow[b]{2}{*}{$\begin{array}{l}\text { Sheath } \\
\text { Air }\end{array}$} & \multirow[b]{2}{*}{ Grounded } & \multicolumn{4}{|c|}{$\mu \mathrm{g} \mathrm{ThO}{ }_{2-}$} \\
\hline & & & & & & & & & Ist Filter & 2nd Filter & Orifice ${ }^{(a)}$ & Walls \\
\hline Th 1 & 10 & Up & $1000^{(b)}$ & 20 & $1 / 10$ & No & No & No & ND & Not used & - & - \\
\hline Th 2 & 10 & Up & $1000^{(b)}$ & 21 & $1 / 10$ & Yes & No & No & ND & Not used & - & - \\
\hline Th 3 & 10 & Down & $1000^{(b)}$ & 22 & 4 & $\mathrm{No}^{(\mathrm{c})}$ & No & No & ND & Not used & - & - \\
\hline Th 4 & 20 & Up & $1000^{(b)}$ & 19 & $1 / 12$ & No & No & No & ND & Not used & - & - \\
\hline Th 5 & 20 & Sideways & 1000 & 19 & $1 / 6$ & Yes & No & No & ND & Not used & - & - \\
\hline Th 6 & 20 & Sideways & 1000 & 19 & 4 & Yes & No & No & $1.0^{(\mathrm{d})}$ & Not used & - & - \\
\hline Th $6 a$ & 20 & Sideways & 1000 & 21 & 5 & Yes & No & No & $10^{(\mathrm{d})}$ & Not used & 50 & - \\
\hline Th 7 & 20 & S1deways & 1000 & 21 & 2 & No & No & No & $1.5^{(\mathrm{d})}$ & $<1.0^{(\mathrm{d})}$ & $<50$ & $1.5^{\text {(d) }}$ \\
\hline Th 8 & 20 & S1deways & 1000 & 21 & 2 & Radial & No & No & $=2.0^{(d)}$ & $3.0^{(d)}$ & $<50$ & $4.0^{(\mathrm{d})}$ \\
\hline Th 9 & 20 & Sideways & 1000 & 23 & $2-1 / 3$ & No & No & Yes & $\mathrm{ND}^{(\mathrm{d})}$ & $\mathrm{ND}^{(\mathrm{d})}$ & $<50$ & $<50$ \\
\hline Th 10 & 20 & Sideways & 1000 & 23 & 2 & Rad1al & Yes & No & ND ${ }^{(d)}$ & Not used & $<50$ & $<50$ \\
\hline Th 11 & 20 & Down & 1000 & 21 & 2 & Radial & Yes & No & $<1.0^{\text {(d) }}$ & Not used & $<50$ & $<50$ \\
\hline Th $11 \mathrm{a}$ & 20 & Down & 1000 & 21 & 2 & Radial & Yes & No & $1.0^{(\mathrm{d})}$ & Not used & $<50$ & $<50$ \\
\hline Th $11 \mathrm{~b}$ & 20 & Down & 1000 & 21 & 2 & Radial & Yes & No & $<1.0^{(\mathrm{d})}$ & Not used & $<50$ & $<50$ \\
\hline Th 12 & 5 & Down & 1000 & 21 & 2 & Radial & Yes & No & $<1.0^{\text {(d) }}$ & Not used & $<50$ & $<50$ \\
\hline Th 13 & 20 & Down & 1000 & 21 & 2 & Axial & Yes & No & $<1.0^{(\mathrm{d})}$ & Not used & $<50$ & $<50$ \\
\hline Th 14 & 50 & Down & 1000 & 21 & 2 & Rad1a1 & Yes & No & $1.5^{(d)}$ & Not used & $<50$ & $<50$ \\
\hline Th 15 & 50 & Sideways & 1000 & 21 & 2 & Radial & Yes & No & $<1.0^{\text {(d) }}$ & Not used & $<50$ & $<50$ \\
\hline Th 16 & 50 & S1deways & 1000 & 24 & 2 & Radial & Yes & No & $35.0^{(d)}$ & Not used & $<50$ & 60 \\
\hline Th 17 & 50 & Down & 1000 & 26 & 2 & No & Yes & No & $85.0^{\text {(d) }}$ & Not used & 96 & 96 \\
\hline Th 18 & 50 & Sideways & 1000 & 26 & 2 & No & Yes & No & $<1.0^{(\mathrm{d})}$ & Not used & $<50$ & $<50$ \\
\hline Th 19 & 50 & Sideways & 1000 & 29 & 2 & Radial & Yes & No & $<1.0^{(\mathrm{d})}$ & Unr used & $<50$ & $<50$ \\
\hline Th 20 & 50 & Up & 1000 & 26 & 2 & Radial & Yes & No & $20.0^{(d)}$ & Not used & $<5$ & $<50$ \\
\hline
\end{tabular}


TABLE 4. SUMMARY OF ROOM TEMPERATURE SIMULANT EXPERIMENTS USING $\mathrm{ThO}_{2}$ (Continued)

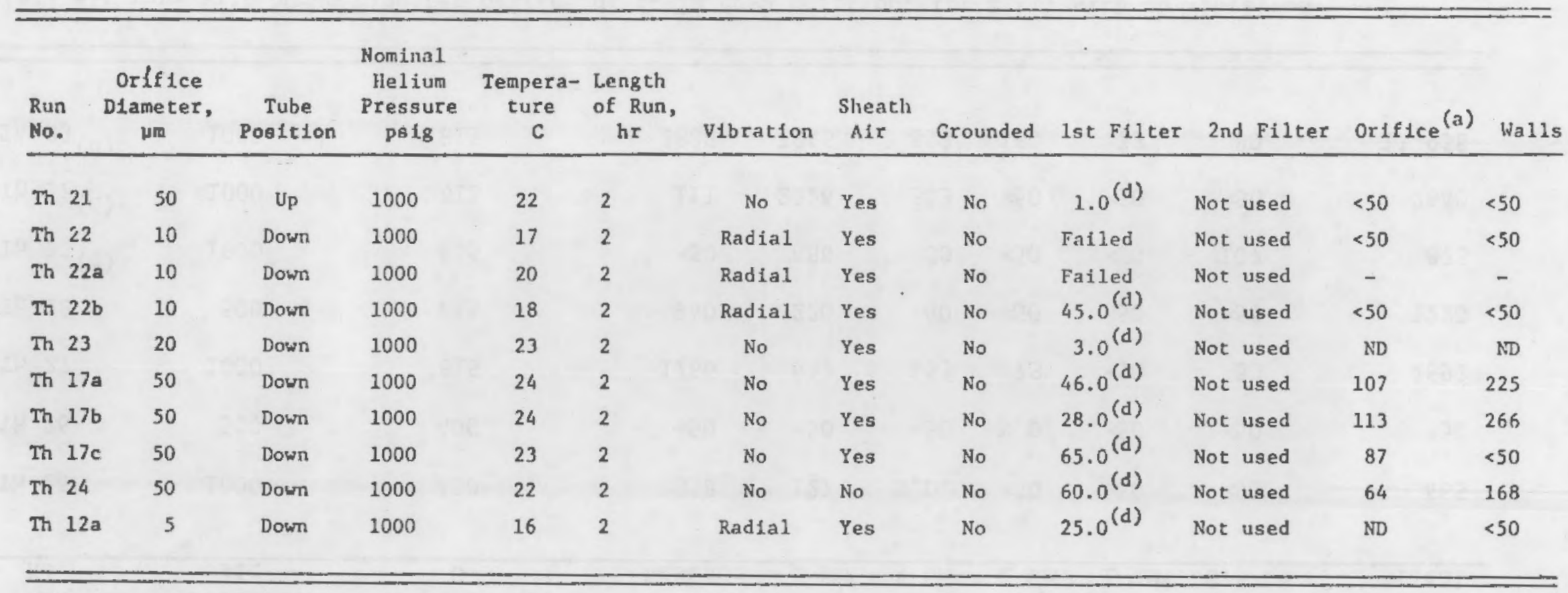

(a) Downstream face.

(b) Pressure was allowed to decay.

(c) The leak tube was vibrated for first 6 months.

(d) These results are based on X-ray fluorescence snalysis. Other data are the results of radioassay alpha counting which has a detection 1 imit of $50 \mu \mathrm{g}$ of $\mathrm{ThO}_{2}$. ND $=$ Not Detected. 
TABLE 5. SUMMARY OF ELEVATED TEMPERATURE SIMULANT RUNS USING CASCADE IMPACTORS (a)

\begin{tabular}{|c|c|c|c|c|c|c|c|c|c|}
\hline \multirow[b]{2}{*}{$\begin{array}{l}\text { Run } \\
\text { No. }\end{array}$} & \multirow[b]{2}{*}{$\begin{array}{l}\text { Pressure, } \\
\text { psig }\end{array}$} & \multirow[b]{2}{*}{$\begin{array}{c}\text { Temperature, } \\
\mathrm{C}\end{array}$} & \multicolumn{6}{|c|}{$\begin{array}{l}\text { Quantity of } \mathrm{ThO}_{2} \text { Detected }^{(\mathrm{b})} \text { for Indicated } \\
\text { Maximum Particle Size, } \mu \mathrm{g}\end{array}$} & \multirow[b]{2}{*}{ Total } \\
\hline & & & $\begin{array}{l}\text { Inlet } \\
\text { Nozzle } \\
\text { Residue }\end{array}$ & $8 \mu \mathrm{m}$ & $4 \mu \mathrm{m}$ & $2 \mu \mathrm{m}$ & $1 \mu \mathrm{m}$ & $0.5 \mu \mathrm{m}$ & \\
\hline Th 25 & 1000 & 400 & 358 & 127 & N.D. & $<50$ & $<0$ & so & 485 \\
\hline Th 26 & 500 & 400 & 50 & $<50$ & $<50$ & N.D. & $<0$ & 50 & $<50$ \\
\hline Th 27 & 1000 & 815 & 1766 & 647 & 153 & 73 & $<50$ & 53 & 2692 \\
\hline Th 28 & 500 & 815 & 940 & 220 & 60 & so & $<50$ & $<50$ & 1220 \\
\hline Th $31^{(c)}$ & 1000 & 815 & $<50$ & 686 & 80 & $<50$ & $<0$ & 107 & 873 \\
\hline Th $32^{\text {(c) }}$ & 1000 & 815 & 111 & 2226 & 303 & $<50$ & $<50$ & $<50$ & 2640 \\
\hline Th $33^{(d)}$ & 1000 & 815 & 2886 & 7846 & 893 & 180 & 73 & 80 & 11,958 \\
\hline
\end{tabular}

(a) All runs with 50- $\mu$ m-diameter orifice disc, in down position, for $2 \mathrm{hr}$, with no vibration.

(b) Data are the results of radioassay alpha counting which has a detection limit of $50 \mu \mathrm{g}$ of $\mathrm{ThO}_{2}$.

(c) The cascade impactor used in this run has a calibrated airflow rate of $131 / \mathrm{min}$. In the other runs, the cascade impactor having a calibrated airflow rate of $1 \mathrm{l} / \mathrm{min}$ was used.

(d) Examination of the orifice after this run showed that the orifice was cracked, which explains why the $\mathrm{ThO}_{2}$ emission was so great. 
was released one time in the sideways and up position with vibration (see Experiments 16 and 20). On the other hand, little or no release occurred in one case of the up position with no vibration (Experiment 21) and numerous runs in the sideways position both with and without vibration (Experiments 7-10, 15, 18, 19).

(5) At $920 \mathrm{psig}$, considerably more $\mathrm{ThO}_{2}$ is emitted through a $50-\mu \mathrm{m}$ orifice at $815^{\circ} \mathrm{C}$ than at $400^{\circ} \mathrm{C}$ (see Th 25 and Th 27).

(6) At $400^{\circ} \mathrm{C}$, more $\mathrm{ThO}_{2}$ is emitted through a $50-\mu \mathrm{m}$ orifice at 920 psig than at 440 psig (see Th 25 and Th 26).

(7) At $815^{\circ} \mathrm{C}$, the data indicate that considerably more $\mathrm{ThO}_{2}$ is emitted at a pressure of 920 psig than at 440 psig.

(8) The use of an impactor with a sheath airflow rate of $131 / \mathrm{min}$, compared to one with a flow rate of $11 / \mathrm{min}$, reduced the stray deposition of $\mathrm{ThO}_{2}$ at $815^{\circ} \mathrm{C}$ with a 50- $\mu \mathrm{m}$ orifice.

(9) A general comparison of the $\mathrm{ThO}_{2}$ leak rate through a 50- $\mu \mathrm{m}$ orffice at room temperature and an internal pressure of 920 psig to the helium leak rate indicates that the emission of $\mathrm{ThO}_{2}$ is less than that of hellum by a factor of about $10^{8}$ on an atom per molecule basis. 



\section{$\underline{\mathrm{PuO}}_{2}$ EXPERIMENTS}

\section{Effect of Experimental Parameters on the}

\section{Size Distribution of the PuO Emission}

The effects of orifice size, helium pressure, leak-tube position, and vibration on the particulate size distribution of the $\mathrm{PuO}_{2}$ emissions are summarized in Table 6 through 8 and illustrated in Figures 15 through 17.

In about half the experiments carried out with $10-$ and $20-\mu \mathrm{m}$ orifices, the average particle size distribution of the leaked $\mathrm{PuO}_{2}$ powder paralleled that of the starting powder. In the case of the $5-\mu \mathrm{m}$ orifice, however, the emission particle size distribution did not parallel that of the starting powder and generally was skewed toward sizes smaller than about $1 \mu \mathrm{m}$. In most of the other runs with $10-$ and $20-\mu \mathrm{m}$ orifices, the particulate distribution was skewed toward sizes greater than 2 um.

Size measurements for several runs indicate a shift to a mean emitted particle size larger than that of the starting powder. In general, this may be accounted for by biased measurements of the initial size distribution. Both the Battelle measurements and the comparable ARHCO measurements were done on suspended and dispersed $\mathrm{PuO}_{2}$ particles. The measurement techniques used would tend to break up or discriminately lose any large agglomerates, thus indicating a smaller than representative mean particle size. Many of the experimental runs may not have this same blasing mechanism. The parametric effects on the emitted $\mathrm{PuO}_{2}$ particle size are discussed below.

- Orifice Size. The effect of orifice size on the average mean particie size of the $\mathrm{PuO}_{2}$ emission is shown in Figure 15. These data indicate that the mean size of the emfssion decreased with a decrease in orifice size at helium pressures of 440 and $920 \mathrm{psig}$. The average mean particle size was $1.5,2.2$, and $5.3 \mu \mathrm{m}$ for the 50-, 10-, and 20- $\mu$ m orifices, respectively. This may be accounted for by increasing shear stress with decreasing orifice size resulting in breakup of agglomerates. Another factor to be considered is the likelihood of the increased 
TABLE 6. PARTICLE SIZE DISTRIBUTION OF $\mathrm{PUO}_{2}$ EMITTED THROUGH A 5- $\mu \mathrm{m}$ ORIFICE AT ROOM TEMPERATURE (a)

\begin{tabular}{|c|c|c|c|c|c|c|c|c|c|c|}
\hline \multirow[b]{2}{*}{$\begin{array}{c}\text { Run } \\
\text { Number }\end{array}$} & \multirow[b]{2}{*}{$\begin{array}{l}\text { Tube } \\
\text { Pogition }\end{array}$} & \multirow{2}{*}{$\begin{array}{c}\text { Nominal } \\
\text { Helium } \\
\text { Pressure } \\
\text { psig }\end{array}$} & \multirow[b]{2}{*}{ vibration } & \multicolumn{6}{|c|}{$\begin{array}{l}\text { Particle Size Distribution } \\
\text { Cumulative Percent }\end{array}$} & \multirow{2}{*}{$\begin{array}{c}\text { Mean } \\
\text { Particle } \\
\text { S1ze, } \\
\mu m \\
\end{array}$} \\
\hline & & & & $\begin{array}{l}<8 \\
\mu \mathrm{m}\end{array}$ & $\begin{array}{l}<4 \\
\mu \mathrm{m} \\
\end{array}$ & $\begin{array}{r}<2 \\
\mu \mathrm{m} \\
\end{array}$ & $\begin{array}{l}<1 \\
\mu m\end{array}$ & $\begin{array}{l}<0.5 \\
\mu \pi\end{array}$ & $\begin{array}{c}<0.25 \\
\text { Hm }\end{array}$ & \\
\hline Starting Powder & - & - & - & 80.0 & 61.0 & 42.0 & 23.0 & 7.0 & 1.7 & 2.5 \\
\hline 28 & Down & 1000 & No & - & 33.4 & 23.2 & 17.5 & 7.8 & 4.9 & 4.7 \\
\hline 29 & SIdewayo & 1000 & Yes & - & 89.1 & 84.9 & 60.2 & 56.4 & 8.5 & 0.57 \\
\hline 30 & Up & 1000 & Yes & - & 87.1 & 86.6 & 82.0 & 30.4 & 4.6 & 0.60 \\
\hline 31 & Down & 500 & Yes & - & 89.7 & 59.8 & 59.8 & 47.7 & 12.2 & 0.88 \\
\hline 32 & S1dewaye & 500 & Yes & - & 86.2 & 68.4 & 56.9 & 8.6 & 6.9 & 1.25 \\
\hline 33 & Up & 500 & Yes & - & 87.7 & 65.8 & 57.5 & 31.5 & 4.1 & 1.07 \\
\hline
\end{tabular}

(a) Al1 runs were for $10 \mathrm{~min}$

(b) Actual pressures vere 920 and 440 psig. 
TABLE 7. PARTICLE SIZE DISTRIBUTION OF PuO 2 EMITTED THROUGH A 10- $\mu \mathrm{m}$ ORIFICE AT ROOM TEMPERATURE (a)

\begin{tabular}{|c|c|c|c|c|c|c|c|c|c|c|}
\hline \multirow[b]{2}{*}{$\begin{array}{c}\text { Run } \\
\text { Number }\end{array}$} & \multirow[b]{2}{*}{$\begin{array}{c}\text { Tube } \\
\text { Position }\end{array}$} & \multirow{2}{*}{$\begin{array}{c}\text { Nomina1 } \\
\text { Hellum } \\
\text { Pressure (b), } \\
\text { psig }\end{array}$} & \multirow[b]{2}{*}{ Vibration } & \multicolumn{6}{|c|}{$\begin{array}{c}\text { Particle Size Distribution, } \\
\text { Cumulative Percent } \\
\end{array}$} & \multirow{2}{*}{$\begin{array}{c}\text { Mean } \\
\text { Particle } \\
\text { S1ze, } \\
\mu \mathrm{m} \\
\end{array}$} \\
\hline & & & & $\begin{array}{l}-88 \\
\text { Hm }\end{array}$ & $\begin{array}{l}<4 \\
\mu \mathrm{m}\end{array}$ & $\begin{array}{l}<2 \\
4 \mathrm{~m}\end{array}$ & $\begin{array}{r}<1 \\
\mu \mathrm{m} \\
\end{array}$ & $\begin{array}{r}<0.5 \\
\mu \mathrm{m} \\
\end{array}$ & $\begin{array}{r}<0.25 \\
\mu \mathrm{m}\end{array}$ & \\
\hline Starting Powder & - & - & - & 80.0 & 61.0 & 42.0 & 23.0 & 7.0 & 1.7 & 2.5 \\
\hline 19 & Down & 1000 & No & - & 91.2 & 89.9 & 89.3 & 87.8 & 84.5 & $<0.25$ \\
\hline 20 & S1deways & 1000 & Yes & - & 80.2 & 63.2 & 43.7 & 37.6 & 8.7 & 1.3 \\
\hline 21 & Up & 1000 & Yes & - & 52.5 & 4.7 & 0.58 & 0.10 & 0 & 4.9 \\
\hline 22 & Down & 500 & Yes & - & 44.5 & 26.1 & 12.6 & 1.9 & 0 & 3.3 \\
\hline 23 & S1deways & 500 & Yes & - & 78.2 & 67.3 & 40.4 & 25.6 & 19.9 & 1.2 \\
\hline 24 & Up & 500 & Yes & - & 90.3 & 15.4 & 8.2 & 4.6 & 0 & 2.0 \\
\hline
\end{tabular}

(a) All runs were for $10 \mathrm{~min}$

(b) Actual pressures were 920 and 440 psig. 
TABLE 8. PARTICLE SIZE DISTRIBUTION OF PUO 2 EMITTED THROUGH A 20- $\mu \mathrm{m}$ ORIFICE AT ROOM TEMPERATURE (a)

\begin{tabular}{|c|c|c|c|c|c|c|c|c|c|c|}
\hline \multirow[b]{2}{*}{$\begin{array}{l}\text { Run } \\
\text { Number (B) }\end{array}$} & \multirow{2}{*}{ Pos1tion } & \multirow{2}{*}{$\begin{array}{c}\text { Nominal } \\
\text { Helium } \\
\text { Pressure } \\
\text { psig } \\
\end{array}$} & \multirow{2}{*}{ Vibration } & \multicolumn{6}{|c|}{$\begin{array}{c}\text { Particle Size Distribution, } \\
\text { Cumulative Percent }\end{array}$} & \multirow{2}{*}{$\begin{array}{c}\text { Average } \\
\text { Mean } \\
\text { Particle } \\
\text { S1ze. } \mathrm{H} \text { 皿 }\end{array}$} \\
\hline & & & & $\begin{array}{l}<8 \\
\mu m\end{array}$ & $<4$ & $\begin{array}{l}<2 \\
\mu m\end{array}$ & . $\quad<1$ & $<0.5$ & $<0.25$ & \\
\hline Starting Powder & - & - & - & 80.0 & 61.0 & 42.0 & 23.0 & 7.0 & 1.7 & 2.5 \\
\hline $\begin{array}{l}\text { Pu } 1, P u \text { 1a } \\
\text { Pu } 2, \mathrm{Pu} 2 \mathrm{a}\end{array}$ & $\begin{array}{l}\text { Down } \\
\text { Down }\end{array}$ & $\begin{array}{l}1000 \\
1000\end{array}$ & $\begin{array}{l}\text { Yes } \\
\text { No }\end{array}$ & - & $\begin{array}{l}60.6 \\
65.9\end{array}$ & $\begin{array}{l}27.5 \\
50.9\end{array}$ & $\begin{array}{r}9.9 \\
40.2\end{array}$ & $\begin{array}{r}3.3 \\
12.1\end{array}$ & $\begin{array}{l}2.0 \\
1.9\end{array}$ & $\begin{array}{l}5.1 \\
2.7\end{array}$ \\
\hline $\begin{array}{l}\text { Pu } 3, P u \\
\text { Pu } 4, P u \\
4 a\end{array}$ & $\begin{array}{l}\text { S1deways } \\
\text { S1deways }\end{array}$ & $\begin{array}{l}1000 \\
1000\end{array}$ & $\begin{array}{l}\text { No } \\
\text { Yes }\end{array}$ & $\overline{-}$ & $\begin{array}{l}46.8 \\
50.2\end{array}$ & $\begin{array}{l}15.9 \\
14.2\end{array}$ & $\begin{array}{l}5.2 \\
6.0\end{array}$ & $\begin{array}{l}3.7 \\
5.1\end{array}$ & $\begin{array}{l}1.0 \\
4.7\end{array}$ & $\begin{array}{l}4.4 \\
7.4\end{array}$ \\
\hline $\begin{array}{l}\text { Pu } 5, P u \text { Sa } \\
\text { Pu } 6, P u \text { 6a, Pu 6b }\end{array}$ & $\begin{array}{l}\text { Up } \\
\text { Up }\end{array}$ & $\begin{array}{l}1000 \\
1000\end{array}$ & $\begin{array}{l}\text { No } \\
\text { Yes }\end{array}$ & - & $\begin{array}{l}61.8 \\
41.4\end{array}$ & $\begin{array}{l}21.7 \\
10.3\end{array}$ & $\begin{array}{l}2.6 \\
1.3\end{array}$ & $\begin{array}{l}0.6 \\
0.12\end{array}$ & $\begin{array}{l}0.2 \\
0.01\end{array}$ & $\begin{array}{l}3.5 \\
5.2\end{array}$ \\
\hline $\begin{array}{l}\text { Pu } 7, P u \quad 7 a \\
\text { Pu } 8, P u \quad 8 a\end{array}$ & $\begin{array}{l}\text { Down } \\
\text { Down }\end{array}$ & $\begin{array}{l}500 \\
500\end{array}$ & $\begin{array}{l}\text { No } \\
\text { Yes }\end{array}$ & $\overline{-}$ & $\begin{array}{l}17.7 \\
28.9\end{array}$ & $\begin{array}{l}2.9 \\
5.8\end{array}$ & $\begin{array}{l}0.5 \\
0.4\end{array}$ & $\begin{array}{l}0.13 \\
0.02\end{array}$ & $\begin{array}{l}0.01 \\
0.01\end{array}$ & $\begin{array}{r}11.0 \\
8.0\end{array}$ \\
\hline $\begin{array}{l}\text { Pu } 9, \text { Pu 9a, Pu 9b } \\
\text { Pu } 14, \text { Pu } 14 a\end{array}$ & $\begin{array}{l}\text { Sideways } \\
\text { S1deways }\end{array}$ & $\begin{array}{l}500 \\
500\end{array}$ & $\begin{array}{l}\text { Yes } \\
\text { No }\end{array}$ & $\begin{array}{c}66.4^{(c)} \\
-\end{array}$ & $\begin{array}{l}52.5 \\
61.1\end{array}$ & $\begin{array}{l}37.4 \\
41.5\end{array}$ & $\begin{array}{l}27.2 \\
29.6\end{array}$ & $\begin{array}{l}16.6 \\
21.2\end{array}$ & $\begin{array}{l}4.1 \\
6.0\end{array}$ & $\begin{array}{l}5.7 \\
4.5\end{array}$ \\
\hline $\begin{array}{l}\text { Pu } 10, \mathrm{Pu} 10 \mathrm{a} \\
\mathrm{Pu} 15, \mathrm{Pu} 15 \mathrm{a}\end{array}$ & $\begin{array}{l}\text { Up } \\
\text { Up }\end{array}$ & $\begin{array}{l}500 \\
500\end{array}$ & $\begin{array}{l}\text { Yes } \\
\text { No }\end{array}$ & $\overline{-}$ & $\begin{array}{l}81.9 \\
80.7\end{array}$ & $\begin{array}{l}12.7 \\
31.7\end{array}$ & $\begin{array}{l}2.6 \\
2.6\end{array}$ & $\begin{array}{l}1.2 \\
0.6\end{array}$ & $\begin{array}{l}0.15 \\
0.25\end{array}$ & $\begin{array}{l}3.4 \\
2.8\end{array}$ \\
\hline $\begin{array}{l}\text { Pu } 11, P u 11 a, P u 11 b \\
\text { Pu } 16\end{array}$ & $\begin{array}{l}\text { Down } \\
\text { Down }\end{array}$ & $\begin{array}{l}\text { Ambient } \\
\text { Amblent }\end{array}$ & $\begin{array}{l}\text { No } \\
\text { Yes }\end{array}$ & - & $\begin{array}{l}74.2 \\
87.5\end{array}$ & $\begin{array}{l}51.5 \\
77.1\end{array}$ & $\begin{array}{l}32.6 \\
65.6\end{array}$ & $\begin{array}{l}16.3 \\
16.7\end{array}$ & $\begin{array}{r}7.2 \\
13.5\end{array}$ & $\begin{array}{l}1.8 \\
0.9\end{array}$ \\
\hline $\begin{array}{ll}\text { Pu } & 12 \\
\text { Pu } & 17\end{array}$ & $\begin{array}{l}\text { S1deways } \\
\text { S1deways }\end{array}$ & $\begin{array}{l}\text { Ambient } \\
\text { Amblent }\end{array}$ & $\begin{array}{l}\text { Yes } \\
\text { No }\end{array}$ & - & $\begin{array}{l}59.4 \\
88.3\end{array}$ & $\begin{array}{l}49.0 \\
72.3\end{array}$ & $\begin{array}{l}17.4 \\
62.8\end{array}$ & $\begin{array}{l}10.1 \\
48.9\end{array}$ & $\begin{array}{r}2.6 \\
37.2\end{array}$ & $\begin{array}{l}2.6 \\
0.5\end{array}$ \\
\hline $\begin{array}{l}\text { Pu } 13 \\
\text { Pu } 18\end{array}$ & $\begin{array}{l}U_{p} \\
U_{p}\end{array}$ & $\begin{array}{l}\text { Amblent } \\
\text { Amblent }\end{array}$ & $\begin{array}{l}\text { Yes } \\
\text { No }\end{array}$ & $\overline{-}$ & $\begin{array}{l}79.3 \\
66.7\end{array}$ & $\begin{array}{l}67.5 \\
59.6\end{array}$ & $\begin{array}{l}22.0 \\
46.5\end{array}$ & $\begin{array}{l}17.2 \\
28.3\end{array}$ & $\begin{array}{l}1.9 \\
0\end{array}$ & $\begin{array}{l}1.6 \\
1.2\end{array}$ \\
\hline
\end{tabular}

(a) 111 runs were for $10 \mathrm{~min}$

(b) Actual pressures were 920 and 440 psig.

(c) Dita for Run Pu 9 only. 


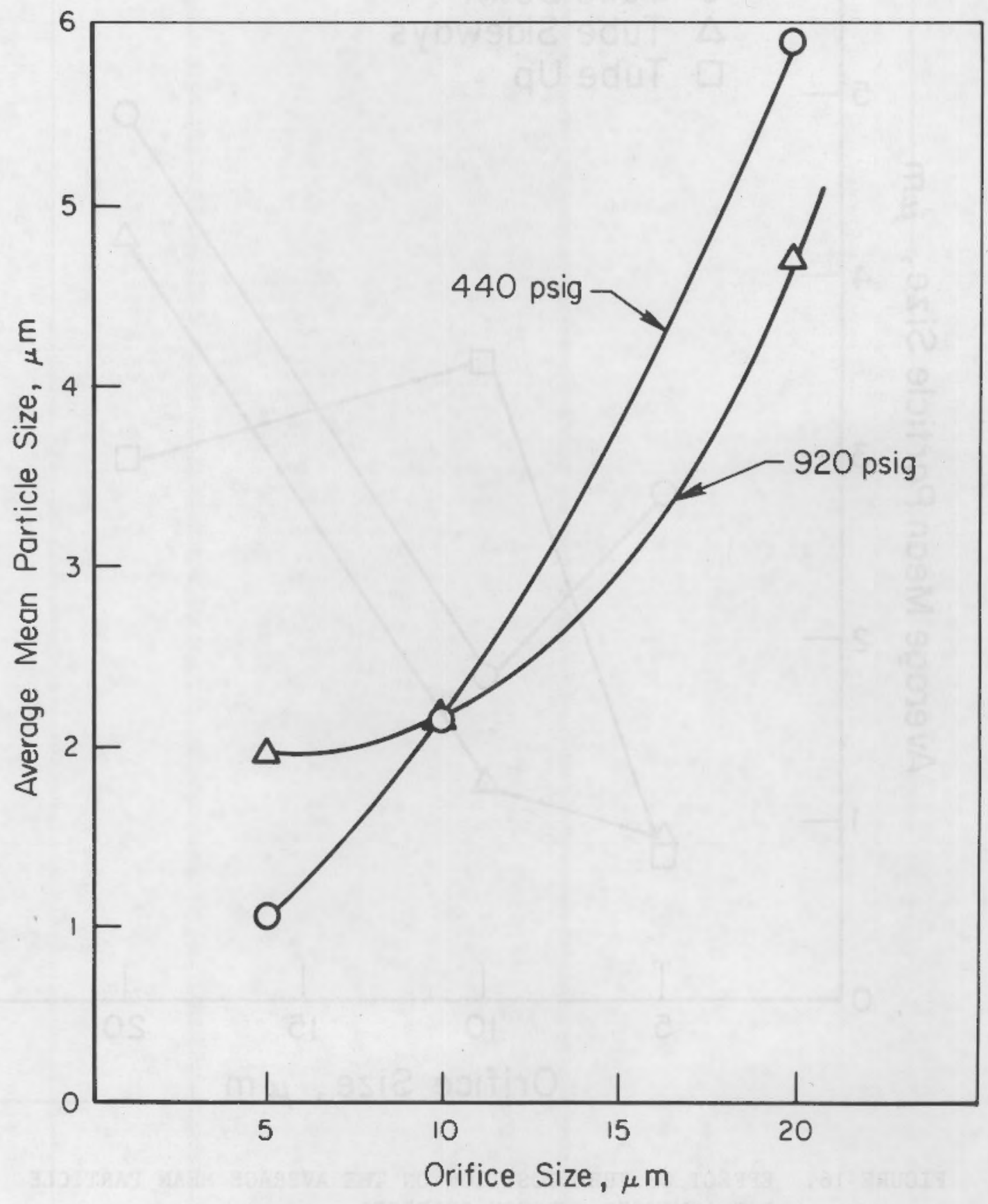

FIGURE 15. EFFECT OF ORIFICE SIZE AND PRESSURE ON THE AVERAGE MEAN PARTICLE SIZE OF EMITTED

$\mathrm{PuO}_{2}$ AT ROOM TEMPERATURE 


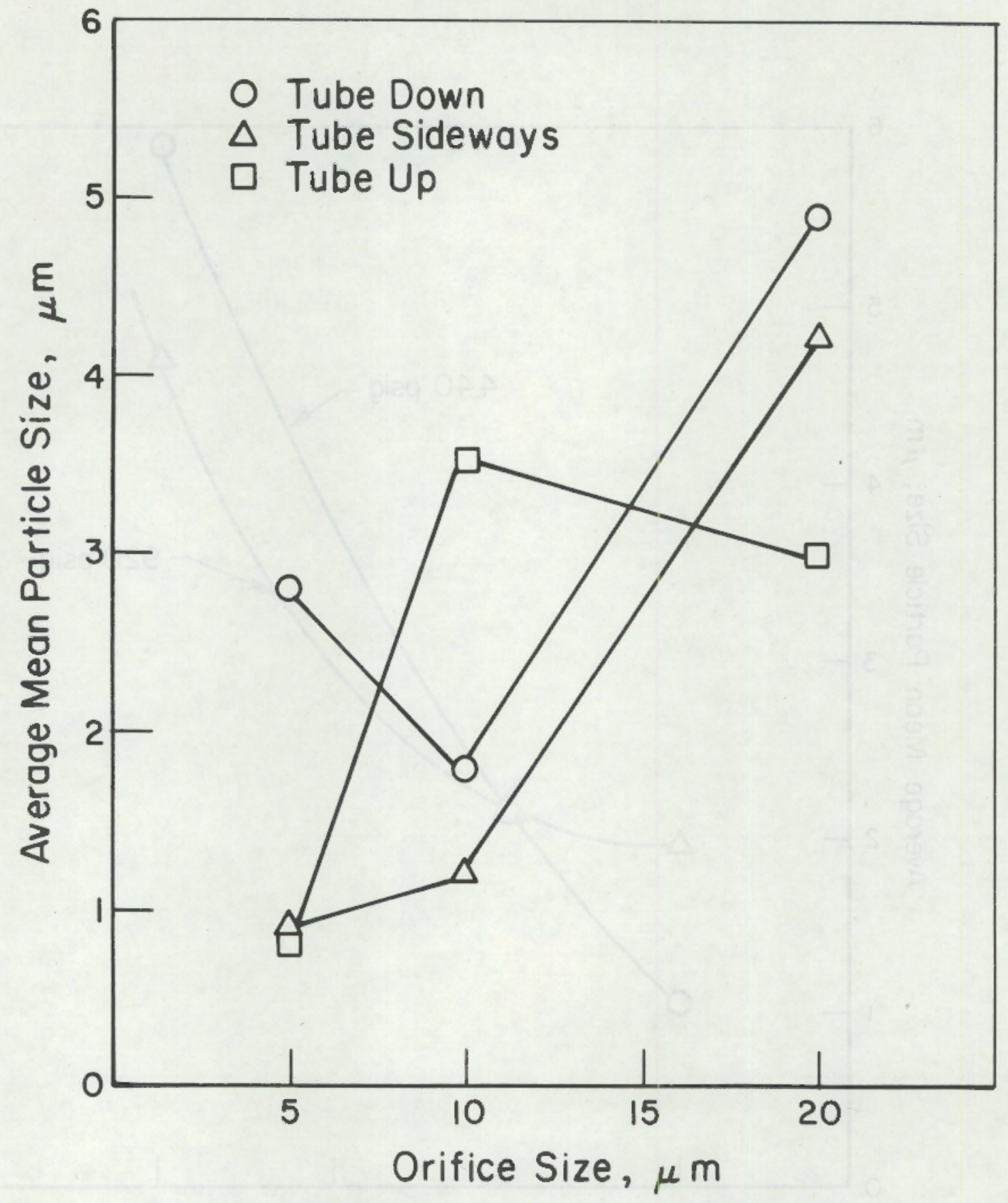

FIGURE 16. EFFEC'T OF TUBE POSITION ON THE AVERAGE MEAN PARTICLE SIZE OF $\mathrm{PuO}_{2}$ EMITTED THROUGH ORIFICES 


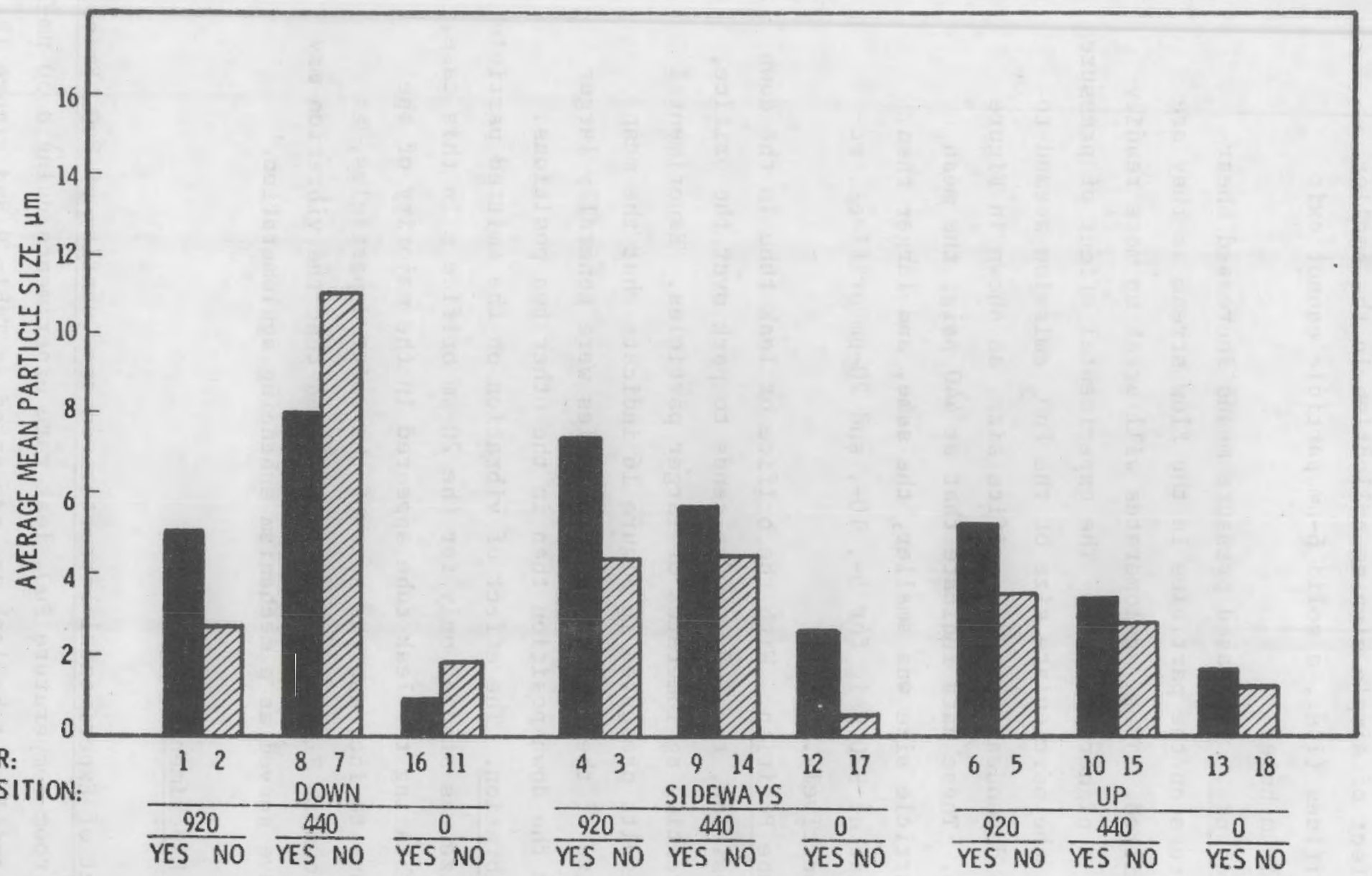

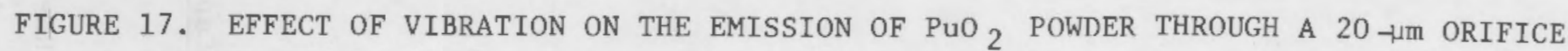


effect of simple sieving collection in the smaller orifices (1.e., a solid 6- $\mu$ m particle cannot exit a 5- $\mu \mathrm{m}$ hole).

- Pressure. Increased pressure means increased shear stress on the particles in the flow stream as they are emitted. Thus, agglomerates will break up more readily at a higher pressure. The experimental effect of pressure on the particulate size of the $\mathrm{PuO}_{2}$ emission seemed to be dependent upon the orifice size, as shown in Figure 15. These data indicate that at $440 \mathrm{psig}$, the mean particle size was smaller, the same, and larger than that at 920 psig for 5-, 10-, and 20- $\mu \mathrm{m}$ orifices, respectively.

- Tube Position. With the orifice or leak tube in the down position, the $\mathrm{PuO}_{2}$ powder tends to pack over the orifice, creating agglomerates of larger particles. Experimental results presented in Figure 16 indicate that the mean size of the emitted $\mathrm{PuO}_{2}$ particles were generally larger in the down position than in the other two positions.

- Vibration. The effect of vibration on the emitted particle size was studied only for the $20-\mu \mathrm{m}$ orifice. In this case, vibrating the leak tube appeared in the majority of the runs to increase the size of the emitted particles, as shown in Figure 17. It is believed that the vibration may have served as a mechanism enhancing agglomeration.

\section{Capillary Leak Experiments}

Effect of Experimental Parameters on the Quantity of $\mathrm{PuO}_{2}$ Emisston. Results of the room-temperature $\mathrm{PuO}_{2}$ leak rate experiments using a 50- $\mu \mathrm{m}-\mathrm{ID}$ x 4.4-cm-long captllary tube leak are presented in Table 9 and Figure 18 . Based on the average total emission of $\mathrm{PuO}_{2}$ powder, the following observations are made. 
TABLE 9. SUMMARY OF PUO 2 LEAK RATE EXPERIMENTS AT ROOM TEMPERATURE USING A 50- $\mu m-I D$ CAPILLARY (a)

\begin{tabular}{|c|c|c|c|c|c|c|c|c|c|c|c|c|c|}
\hline \multirow[b]{2}{*}{$\begin{array}{c}\begin{array}{c}\text { Run } \\
\text { Number }\end{array} \\
\end{array}$} & \multirow[b]{2}{*}{$\begin{array}{c}\text { Tube } \\
\text { Position }\end{array}$} & \multirow[b]{2}{*}{$\begin{array}{c}\text { Pressure, } \\
\text { pg1g }\end{array}$} & \multirow[b]{2}{*}{ Vibration } & \multicolumn{4}{|c|}{ Quantiky of $\mathrm{PuO}_{2}$ Detected for } & \multicolumn{4}{|c|}{ Indicated Particle Size, $\mu \mathrm{g}{ }^{(b)}$} & \multirow[b]{2}{*}{ Total (c) } & \multirow{2}{*}{$\begin{array}{l}\text { Helium } \\
\text { Leak } \\
\text { Rate, } \\
\text { cc/sec }\end{array}$} \\
\hline & & & & $\begin{array}{l}\text { Inlet } \\
\text { Nozzle }\end{array}$ & $\begin{array}{l}>4 \\
\mu \mathrm{m} \\
\end{array}$ & $\begin{array}{l}4-2 \\
\mu \mathrm{m} \\
\end{array}$ & $\begin{array}{l}2-1 \\
\mu \mathrm{m}\end{array}$ & $\begin{array}{c}1-0.5 \\
\mu \mathrm{m} \\
\end{array}$ & $\begin{array}{c}0.5-0.25 \\
\mu \mathrm{m}\end{array}$ & $\begin{array}{c}<0.25 \\
\mu \mathrm{m}\end{array}$ & $\begin{array}{c}\text { F12ter, } \\
\mu \mathrm{m}\end{array}$ & & \\
\hline Pu 39 & Down & 1000 & No & 0.0076 & 0.0006 & 0.0003 & 0.0013 & 0.0004 & 0.0006 & 0.0001 & - & 0.0073 & 0.87 \\
\hline Pu $39 a^{(e)}$ & Down & 1000 & No & 0.0025 & - & - & - & - & - & - & 0.0005 & -0.0011 & 14.1 \\
\hline Pu 40 & Up & 1000 & Yes & 0.0032 & 0.0019 & 0.0025 & 0.0013 & 0.0069 & 0.0006 & - & - & 0.0129 & 27.4 \\
\hline $\mathrm{Pu} 40 \mathrm{a}^{(\mathrm{e})}$ & Up & 1000 & Yes & 0.0050 & - & - & - & - & - & - & 0.0001 & 0.001 & 26.1 \\
\hline Pu 41 (0) & Down & 500 & Yes & 0.0057 & 0.0025 & 0.0000 & 0.0006 & 0.0005 & 0.0004 & 0.0003 & - & 0.0060 & 3.9 \\
\hline $\mathrm{Pu} 41 \mathrm{a}$ (e) & Down & 500 & Yes & 0.0013 & - & - & - & - & - & - & 0.0013 & -0.0015 & 0.86 \\
\hline Pu 42 (a) & Up & 500 & Yes & 0.0025 & - & - & - & - & - & - & - & 0.0041 & 4.8 \\
\hline Pu $42 a(e)$ & Up & 500 & Yes & 0.0139 & - & - & - & - & - & - & 0.0001 & 0.0098 & 5.6 \\
\hline $\mathrm{Pu} 43^{(\mathrm{e})}$ & Down & Aubient & No & 0.0063 & - & - & - & - & - & - & 0.0397 & 0.0419 & - \\
\hline Pu $43 a$ (e) & Down & Ambient & No & 0.0044 & - & - & - & - & - & - & 0.0006 & 0.0010 & - \\
\hline $\mathrm{Pu} 44^{(\mathrm{e})}$ & Up & Ambient & Yes & 0.0013 & - & - & - & - & - & - & 0.0025 & -0.0000 & - \\
\hline Pu $44 a^{(e)}$ & Up & Amblent & Yes & 0.0032 & - & - & - & - & - & - & 0.0013 & 0.0003 & - \\
\hline
\end{tabular}

(a) A11 runs were for $10 \mathrm{~min}$

(b) Based on the specific activity of $0.096 \mathrm{Cl} / \mathrm{g}$ for Pu.

(c) The average box background for these experiments was $0.0041 \mu \mathrm{g}$ Pu0 for a ten-min collection. This quantity was subtracted from the total enission, but not from the emissions for each size range.

(d) The leak rate was determined by the pressure decay method at the midpoint of the run. In an empty leak tube, the He leak rate was 0.8 and $0.3 \mathrm{cc} / \mathrm{sec}$ at 920 and $440 \mathrm{psig}$, respectively. Thus, leak rates higher than those are presumably Indlcative of leak in He supply line to the leak tube.

(e) The sizing stages of the cascade impactor were replaced with a tube and all of the Puo 2 emission was collected on the final filter located at the entrance end. 


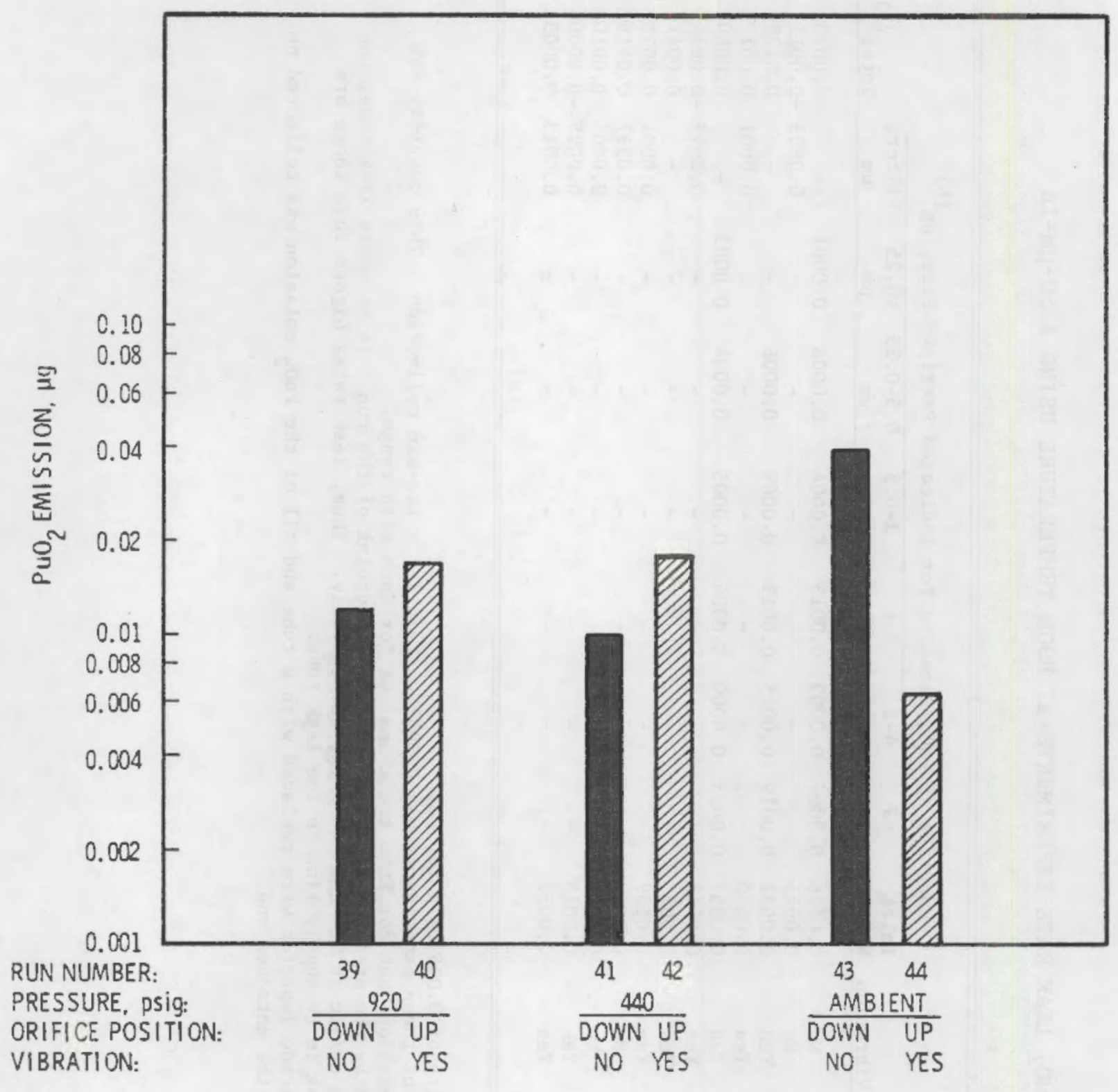

FIGURE 18. EFFECT OF EXPERIMENTAL CONDITIONS ON THE AVERAGE EMISSION OF $\mathrm{PuO}_{2}$ POWDER THROUGH A 50- $\mu \mathrm{m}$-ID CAPILLARY AT ROOM TEMPERATURE 
- The greatest emission occurred at ambient pressure with the leak tube in the down position. The $\mathrm{He}: \mathrm{PuO}_{2}$ leak rate ratio was $4.9 \times 10^{2}$ in this experiment.

- The $\mathrm{PuO}_{2}$ emission was slightly greater with the leak tube in the up position at helium pressures of 920 and 440 psig. However, at ambient pressure, the emission was greater in the down position.

- In general, pressure appeared to have little effect on the $\mathrm{PuO}_{2}$ emission.

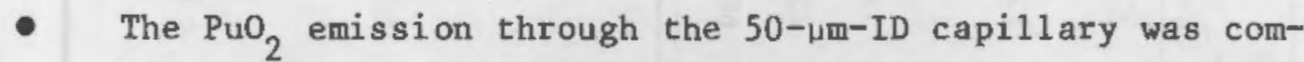
parable to that through the $5-\mu m$ orifice at helium pressures of 920 and 400 psig and at ambient pressure. Helium leak rates were similarly comparable under all conditions. Effect of Experimental Parameters on the Size Distribution of the $\mathrm{PuO}_{2}$ Emission. Particle-size data on the room-temperature capillary leak experiments are presented in Table 10 and Figure 19. The average mean particle size of $\mathrm{PuO}_{2}$ emission was about $1.3 \mu \mathrm{m}$ and $2.3 \mu \mathrm{m}$ at helium pressures of 920 and $440 \mathrm{psig}$, respectively. The overall average mean particle size was $1.8 \mathrm{\mu m}$,

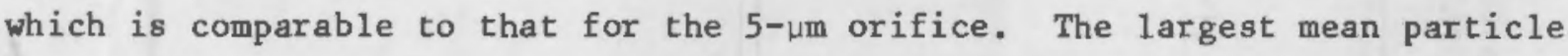
size of leaked particles was $3.9 \mu \mathrm{m}$, which occurred at $440 \mathrm{psig}$ with the leak tube down and vibrated.

The particle-size distribution of the emission for Run Pu 41 was similar to that of the starting powder. For the other runs, there was a general displacement of the size distribution toward emission of smaller particles. Run Pu 42 showed an unusually large percentage of particles smaller than $0.25 \mu \mathrm{m}$. 
TABLE 10. PARTICLE SIZE DISTRIBUTION OF PuO 2 EMISSION THROUGH A $50-\mu \mathrm{m} \times 4.4-\mathrm{cm}-$ LONG CAPILLARY TUBE AT ROOM TEMPERATURE(a)

\begin{tabular}{|c|c|c|c|c|c|c|c|c|c|c|}
\hline \multirow[b]{2}{*}{ Number } & \multirow[b]{2}{*}{ Position } & \multirow{2}{*}{$\begin{array}{c}\text { Nominal } \\
\text { Helium } \\
\text { Pressure (b) } \\
\text { psig }\end{array}$} & \multirow[b]{2}{*}{ Vibration } & \multicolumn{6}{|c|}{$\begin{array}{c}\text { Particle Size Distribution, } \\
\text { Cumulative Percent }\end{array}$} & \multirow{2}{*}{$\begin{array}{c}\text { Mean } \\
\text { Particle } \\
\text { Size } \\
\end{array}$} \\
\hline & & & & $<8$ & $<4$ & $<2$ & $<1$ & $<0.5$ & $<0.25$ & \\
\hline Starting Powder & - & - & - & 80.0 & 61.0 & 42.0 & 23.0 & 7.0 & 1.7 & 2.5 \\
\hline Pu 39 & Down & 1000 & No & - & 78.3 & 70.3 & 31.9 & 21.0 & 2.9 & 1.3 \\
\hline $\mathrm{Pu} 40$ & Up & 1000 & Yes & - & 86.0 & 68.1 & 59.5 & 6.5 & 0 & 1.2 \\
\hline Pu 41 & Down & 500 & Yes & - & 44.0 & 43.5 & 25.1 & 14.7 & 6.8 & 3.9 \\
\hline Pu 42 & Up & 500 & Yes & - & 84.3 & 65.9 & 60.7 & 45.0 & 41.5 & 0.6 \\
\hline
\end{tabular}

(a) Ali runs were for $10 \mathrm{~min}$

(b) Actual pressures were 920 and 440 psig. 


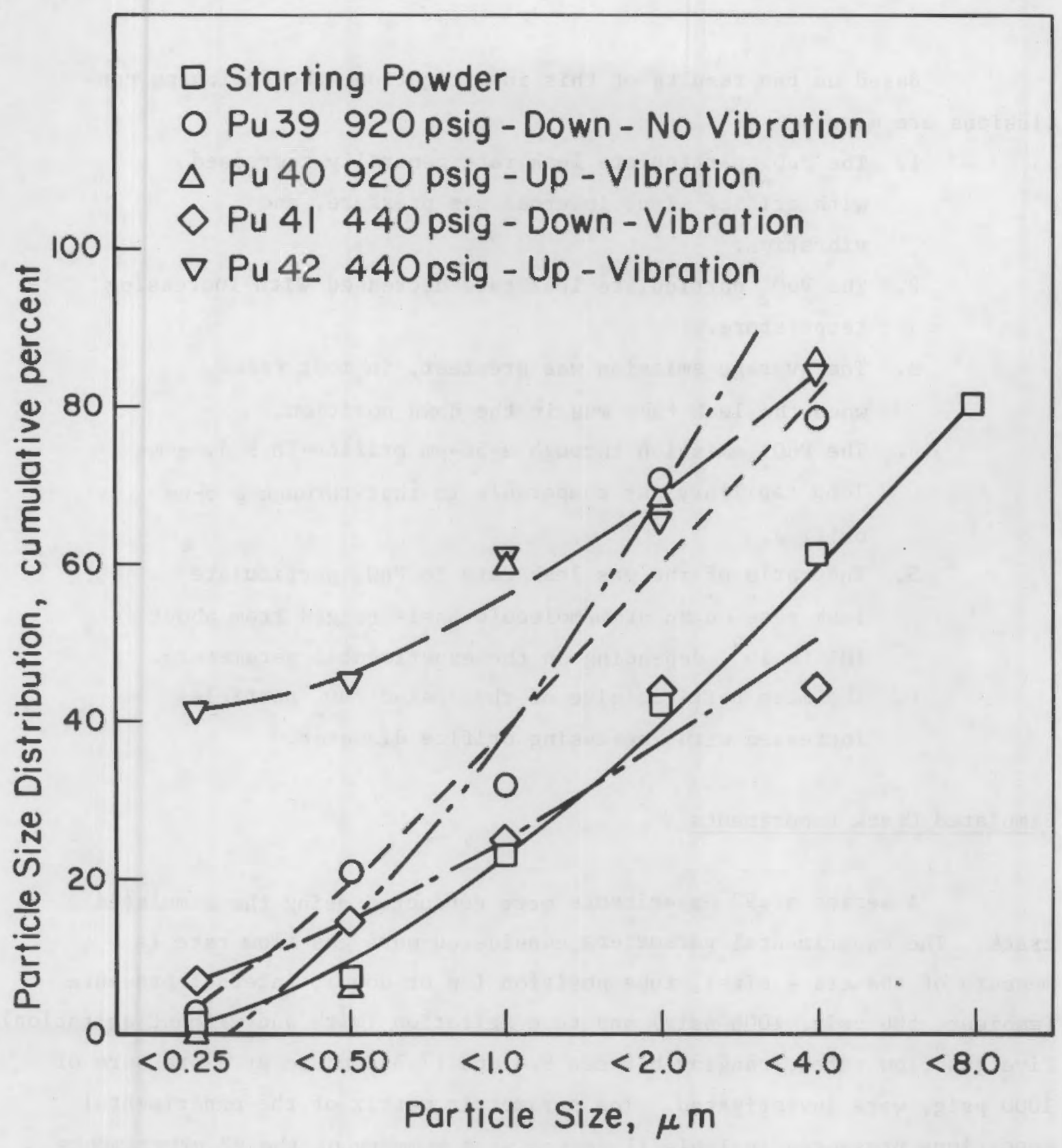

FIGURE 19. PARTICLE SIZE DISTRIBUTION OF PuO 2 EMISSION THROUGH A 50- $\mu m-I D ~ x$ $4.4-\mathrm{cm}$-LONG CAPILLARY TUBE AT ROOM TEMPERATURE 
Based on the results of this investigation, the following conclusions are made:

1. The $\mathrm{PuO}_{2}$ particulate leak rate generally increased with orifice size, internal gas pressure, and vibration.

2. The $\mathrm{PuO}_{2}$ particulate leak rate decreased with increasing temperature.

3. The average emission was greatest, in most cases, when the leak tube was in the down position.

4. The $\mathrm{PuO}_{2}$ emission through a $50-\mu \mathrm{m}$ orifice-ID $\times 4.4-\mathrm{cm}-$ long capillary was comparable to that through a $5-\mu \mathrm{m}$ orifice.

5. The ratio of the gas leak rate to $\mathrm{PuO}_{2}$ particulate leak rate on an atom/molecule basis ranged from about $10^{3}$ to $10^{9}$, depending on the experimental parameters.

6. The mean particle size of the leaked $\mathrm{PuO}_{2}$ particles increased with increasing orifice diameter.

Simulated Crack Experiments

A series of 92 experiments were conducted using the simulated crack. The experimental parameters considered were gas flow rate (a measure of the crack size), tube position (up or down), internal pressure (ambient, $500 \mathrm{psig,} 1000 \mathrm{psig}$ ) and tube agitation (with and without agitation). Five gas flow rates, ranging between 9.8 and $17.3 \mathrm{scc} / \mathrm{sec}$ at a pressure of 1000 psig, were investigated. The parametric matrix of the experimental conditions presented in Table 11 serves as a summary of the 92 experiments conducted.

The results of the experiments are presented in Appendix B, Tables B1 through B5.

Inspection of the data tables reveals the extreme variability exhibited by the data. This observation is more clearly demonstrated in Table 12 which presents the arithmetic mean value of the $\mathrm{PuO}_{2}$ emissions 
TABLE 11. PARAMETRIC MATRIX OF $\mathrm{PuO}_{2}$ EXPERIMENTS

USING THE SIMULATED CRACK

\begin{tabular}{|c|c|c|c|c|c|c|c|c|}
\hline \multirow{2}{*}{$\begin{array}{c}\text { Leak }^{(a)} \\
\text { Rate } \\
(\mathrm{scc} / \mathrm{sec})\end{array}$} & \multirow[b]{2}{*}{ Vibration } & \multirow{2}{*}{$\begin{array}{l}\text { Pressure: } \\
\text { Position: }\end{array}$} & \multicolumn{2}{|c|}{ Ambient } & \multicolumn{2}{|c|}{$500 \mathrm{pstg}$} & \multicolumn{2}{|c|}{$1000 \mathrm{psig}$} \\
\hline & & & $\overline{\mathrm{Up}}$ & $\overline{\text { Down }}$ & $\overline{\mathrm{Up}}$ & Down & $\overline{\mathrm{Up}}$ & Down \\
\hline 9.8 & $\mathrm{Y}$ & & 2 & 2 & 3 & 2 & 2 & - \\
\hline 9.8 & $\mathrm{~N}$ & & - & 2 & - & - & 2 & 2 \\
\hline 11.4 & $\mathbf{Y}$ & & 2 & - & 3 & 2 & 2 & - \\
\hline 11.4 & $\mathrm{~N}$ & & - & 2 & - & - & 2 & 2 \\
\hline 11.6 & $\mathrm{Y}$ & & 2 & - & 2 & 4 & 4 & - \\
\hline 11.6 & $\mathrm{~N}$ & & - & 2 & - & - & 4 & 6 \\
\hline 13.2 & $\mathrm{Y}$ & & 2 & - & 4 & 2 & 2 & - \\
\hline 13.2 & $\mathrm{~N}$ & & - & 4 & - & - & 4 & 2 \\
\hline 17.3 & $\mathrm{Y}$ & & 2 & - & 2 & 2 & 4 & - \\
\hline 17.3 & $\mathrm{~N}$ & & - & 2 & - & - & 2 & 2 \\
\hline
\end{tabular}

(a) Leak rate at 1000 psig using pressure decay method; determined prior to runs. 
TABLE 12. ARITHMETIC MEAN VALUE OF PuO POWDER LEAKED AND THE STANDARD DEVIATION FOR EACH EXPERIMENTAL CONDITION USING THE SIMULATED CRACK CONFIGURATION (MEAN/STANDARD DEVIATION) (IN NEAREST $\mathrm{ng}$ )

\begin{tabular}{|c|c|c|c|c|c|c|c|c|}
\hline \multirow{2}{*}{$\begin{array}{c}\text { Leak } \\
\text { Rate } \\
(\mathrm{scc} / \mathrm{sec})\end{array}$} & \multirow[b]{2}{*}{ Vibration } & \multirow{2}{*}{$\begin{array}{l}\text { Pressure: } \\
\text { Position: }\end{array}$} & \multicolumn{2}{|c|}{ Amblent } & \multicolumn{2}{|c|}{500 psig } & \multicolumn{2}{|c|}{1000 psig } \\
\hline & & & Up & Down & Up & Down & Up & Down \\
\hline 9.8 & $\mathrm{Y}$ & & $3 / 3$ & $-1 / 0$ & $1 / 2$ & $66 / 44$ & $5 / 5$ & - \\
\hline 9.8 & $\mathrm{~N}$ & & - & $0 / 1$ & - & - & $4 / 0$ & $2 / 1$ \\
\hline 11.4 & $\mathrm{Y}$ & & $1 / 0$ & - & $0 / 0$ & $0 / 0$ & $-1 / 0$ & - \\
\hline 11.4 & $\mathrm{~N}$ & & - & $-1 / 1$ & - & - & $-1 / 1$ & $-1 / 0$ \\
\hline 11.6 & $\mathrm{Y}$ & & $-1 / 0$ & - & $-2 / 1$ & $469 / 851$ & $441 / 872$ & - \\
\hline 11.6 & $\mathrm{~N}$ & & - & $0 / 1$ & - & - & $180 / 265$ & $5955 / 14127$ \\
\hline 13.2 & $\mathrm{Y}$ & & $-I / 1$ & - & $1 / 2$ & $570 / 114$ & $5 / 2$ & - \\
\hline 13.2 & $\mathrm{~N}$ & & - & $37 / 73$ & - & - & $28 / 50$ & $41 / 17$ \\
\hline 17.3 & $Y$ & & $0 / 0$ & - & $5 / 1$ & $553 / 134$ & $57 / 97$ & - \\
\hline 17.3 & $\mathrm{~N}$ & & - & $9 / 10$ & - & - & $42 / 21$ & $24 / 2$ \\
\hline
\end{tabular}


and the standard deviation for each experimental condition. For many but not all conditions the variability is such that the standard deviation assoctated with a series of experiments carried out under identical conditions is greater than the mean value of those experiments. Such extreme variability would be expected to mask all but the most obvious parametric effects.

A visual examination of the data in Tables B1 through B5 or the average values of Table 12 reveals no consistent pattern of dependence of the quantity of $\mathrm{PuO}_{2}$ emitted on the experimental parameters. For the sake of thoroughness, the data were analyzed by standard test of statistical significance. Prior to the analysis the data were transformed $10 \mathrm{~g}-$ arithmically to the form

$$
z=\log (1000 x+10)
$$

where $\mathrm{X}$ is the total $\mathrm{PuO}_{2}$ emission in mlcrograms. This transformation was suggested by a plot of the standard deviation versus the average PuO 2 emission for a given set of conditions which demonstrated that the standard deviation is directly proportional to the quantity of $\mathrm{PuO}_{2}$ emitted. This transformation served to somewhat stabilize the varlability of the data and reduce the skewness. Although this transformation was not ideal, attempts to provide a better one were unsuccessful. This logarithmic transformation was used for the subsequent data analysis. Using standard tests of statistical significance, the data were examined for effects of the internal gas pressure, leak size, tube position, and agitation of the powder. Within the limits imposed by the varfability of the data no consistent parametric dependence could be discerned.

$\frac{\text { Parametric Dependence of } \mathrm{PuO}_{2}}{\text { Emissions Through Orifices }}$

A total of 250 experiments were conducted using orifices ranging from 5 through $50 \mu \mathrm{m}$ in diameter. The experimental parameters investigated were orifice size, internal pressure, tube position and powder agitation. The results of these experiments and the actual conditions under which they were conducted are presented in Tables B6 through B17 of Appendix B. 
The experimental results for the orifice experiments exhibit an extreme degree of variability. Repeated runs (1.e., several runs conducted under identical experimental conditions) of ten differed from one another by several orders of magnitude. This varlability is probably a consequence of the small quantity of $\mathrm{PuO}_{2}$ being emitted. In many cases, the differences observed between a series of identical runs are no greater than what would be expected by the addition or subtraction of a single large particle of plutonia powder. In fact, in some cases, the entire quantity of $\mathrm{PuO}_{2}$ detected could be attributed to one powder particle. In the original powder, 20 percent of the particles had diameters of greater then $8 \mu \mathrm{m}$, which corresponds to a $\mathrm{PuO}_{2}$ mass of approximately 25 nanograms per particle. It is easy to see how one particle could affect the emission. When working with such small numbers of particles, it is not surprising to see the severe variability of the data.

Due to the small quantities of $\mathrm{PuO}_{2}$ emitted and the high sensitivity of the radioassay technique, it would not be expected that the reproducibility of the data would be exact. However, these data may be evidence that the $\mathrm{PuO}_{2}$ emissions are influenced by some undefined factor. One such factor considered is possible plugging and unplugging of the leak during the course of a run. A digital flow meter was installed in the system to study this. Flow rate data were accumulated during each run and examined for changes of flow. The data showed that although there was considerable fluctuation in the flow rates between runs, there was no significant change during any given run. The indications were that those incidents which resulted in flow rate changes had to occur during the initial pressurization or final depressurization of the leak tube, when the flow rate cannot be measured.

\section{Background Subtraction}

The containment box background measurements appear to exhibit a lack of reproductbility similar to that observed for the actual run measurements. It was therefore felt that it would be more appropriate to use an 
average value for the background rather than to rely upon a single measurement associated with a series of runs. The earlier experiments Pu 1 through Pu 36 exhibited a consistently higher background than the later runs $\mathrm{Pu} 82$ through $\mathrm{Pu} 131$ so the background averages used for these two sets of runs differed. For the earlier runs, the background for the runs was 0.0030 $\mu \mathrm{g}$, while the latter set of background was $0.0013 \mu \mathrm{g}$ with subtraction was greater than that associated with the counting of the $\mathrm{PuO}_{2}$ emitted. Consequently, only the background error will contribute significantly to the error associated with the net total emissions.

\section{Collection Time Dependence}

To determine the effects of the collection time, a series of experiments with varying collection times was conducted. The time effect was investigated for both $20-\mu \mathrm{m}$ and $50-\mu \mathrm{m}$-diameter orifices. The results of these experiments are included in the tables of Appendix B. For the 20-um-diameter orifices, the experiments were all conducted at $1000 \mathrm{psig}$ pressure, in the upright position and with no vibration. The $20-\mu m$-orifice runs were compared and their' collection times were $\mathrm{Pu} 107$ through $\mathrm{Pu}$ 107n (10 $\mathrm{min}$ ), Pu 108 through Pu 108d (60 min), Pu 109 through Pu 109d ("zero time") and $\mathrm{Pu} 110$ through Pu $110 \mathrm{~d}(120 \mathrm{~min})$. For the $50-\mu \mathrm{m}$-diameter orifice runs, the conditions were 1000 psig, up position, with vibration. These experiments and collection times are labelled $\mathrm{Pu} 119$ through $\mathrm{Pu} 119 \mathrm{e}$ (10 min), Pu 128 through Pu 128d ("zero time"), Pu 129 through Pu 129d (60 min). The "zero time" designation refers to those experiments conducted with the collection time and the time during which the tube is fully pressurized kept to the most practical minimum. This was accomplished by rapidly pressurizing the leak tube and immediately after achieving the desired pressure, shutting off the helium supply and releasing the pressure through a bleed valve. The entire operation requires somewhat less than 1 min.

Prior to analysis, the data were subjected to a logarithmic transformation designed to eliminate negative numbers and zeros and help minimize skewness and stabilize the variance. The transformed data were 
examined for effects of the collection time using standard tests for statistical significance ( $F$ - test, Students t-test). No consistent run time dependence was observed for either orifice size examined.

\section{Parametric Dependency of Orifice Data}

The experiments may be considered as consisting of two sets of data. The two sets were both conducted using essentially identical equipment (only minor changes made between sets) but were separated by an extended time period. The first set (Phase I) was completed early in the experimental program. The second set (Phase II) was conducted several months later, and was designed to augment the origina1 exprimental design in a way that would either confirm or refute the existence of certain effects that were suggested by the results of the Set I experiments. It was also anticipated that the Phase II data would improve the estimate of experimental error computed from the Phase I data.

The orifice experiments conducted, including those of both Phase $I$ and Phase II, is summarized in Tables 13 and 14. Inasmuch as each experimental run is expensive and time consuming, the number of runs had to be limited to the most important treatment combinations. This accounts for the empty cells in the matrix, which preclude a simple, unified analysis.

Because of the extreme heteroschedasticity (lack of uniform variability as experimental conditions change) and positive skewness (a concentration of low values, along with a few extremely large values) exhibited by the raw data, a transformation of the data seemed advisable in order to make use of standard parametric statistical techniques.

The mean and standard deviation of various cells in the matrix of experimental treatments were noticeably correlated, which led us to consider a logarithmic transformation. The difficulty of dealing with cumbersome decimal fractions and negative observations (which cannot be converted to logarithms) was eliminated by increasing the product $1000 \mathrm{x}$ 10 , for every $x$. The transformation equation thus took the form 
TABLE 13. PARAMETRIC MATRIX OF PuO 2 LEAK RATE EXPERIMENTS USING STANDARD ORIFICES DATA SET

\begin{tabular}{|c|c|c|c|c|c|c|c|c|c|c|c|}
\hline \multirow{2}{*}{$\begin{array}{l}\text { Orifice } \\
\text { Size }\end{array}$} & \multirow[b]{2}{*}{ Vibration } & \multirow{2}{*}{$\begin{array}{l}\text { Pressure: } \\
\text { Positon: }\end{array}$} & \multicolumn{3}{|c|}{ Ambtent } & \multicolumn{3}{|c|}{500 psig } & \multicolumn{3}{|c|}{1000 psig } \\
\hline & & & Up & Down & S1deways & $U_{p}$ & Down & S1deways & Up & Down & Sideways \\
\hline 5 & $\mathbf{Y}$ & & 2 & - & 2 & 2 & 2 & 2 & 2 & - & 2 \\
\hline 5 & $\mathrm{~N}$ & & - & 2 & - & - & - & - & - & 4 & - \\
\hline 10 & $\mathrm{Y}$ & & 2 & - & 2 & 2 & 2 & 2 & 3 & - & 2 \\
\hline 10 & N & & - & 2 & - & - & - & - & - & 2 & - \\
\hline 20 & $\mathrm{Y}$ & & 2 & 2 & 3 & 4 & 2 & 3 & 3 & 2 & 2 \\
\hline 20 & $\mathrm{~N}$ & & 2 & 3 & 2 & 2 & 2 & 2 & 2 & 2 & 2 \\
\hline
\end{tabular}


TABLE 14. PARAMETRIX MATRIX OF PuO 2 LEAK RATE EXPERIMENTS USING STANDARD ORIFICES DATA SET II

\begin{tabular}{|c|c|c|c|c|c|c|c|c|c|c|c|}
\hline \multirow{2}{*}{$\begin{array}{l}\text { Orifice } \\
\text { Size }\end{array}$} & \multirow[b]{2}{*}{ Vibration } & \multirow{2}{*}{$\begin{array}{l}\text { Pressure: } \\
\text { Positon : }\end{array}$} & \multicolumn{2}{|c|}{ Ambient } & \multicolumn{2}{|c|}{$500 \mathrm{psig}$} & \multicolumn{3}{|c|}{1000 psig } & \multicolumn{2}{|c|}{$1250 \mathrm{psig}$} \\
\hline & & & Up & Down & $\overline{\mathrm{Up}}$ & Down & $\overline{\mathrm{Up}_{\mathrm{p}}}$ & Down & Sideways & $\overline{\mathrm{Up}}$ & Down \\
\hline 5 & $\mathbf{Y}$ & & - & 4 & - & 2 & - & 2 & - & - & - \\
\hline 5 & $\mathbf{N}$ & & 2 & - & - & 6 & - & 6 & - & - & - \\
\hline 8 & $\mathbf{Y}$ & & - & - & - & - & 4 & 4 & 4 & 2 & 2 \\
\hline 8 & $\mathbf{N}$ & & - & - & - & - & - & 4 & - & - & 4 \\
\hline 10 & $\mathbf{Y}$ & & - & - & - & 4 & - & - & - & - & - \\
\hline 10 & $\mathbf{N}$ & & - & - & 4 & 4 & - & - & - & - & - \\
\hline 20 & $\mathrm{Y}$ & & 2 & 2 & - & - & 4 & 4 & - & - & - \\
\hline 20 & $\mathbf{N}$ & & 2 & 2 & - & - & 32 & 2 & - & - & - \\
\hline 50 & $\mathrm{Y}$ & & 2 & 2 & 4 & 5 & 20 & 5 & - & 4 & 4 \\
\hline 50 & $\mathbf{N}$ & & - & - & 2 & 2 & 15 & - & - & 2 & 2 \\
\hline
\end{tabular}


where

$$
z=\log _{10}(1000 x+10)
$$

$$
\begin{aligned}
& \mathrm{X}=\text { net quantity of } \mathrm{PuO}_{2} \\
& \mathrm{Z}=\text { new vartate after transformation }
\end{aligned}
$$

Although this transformation was somewhat effective in stabilizing the variance and reducing the skewness, it fell short of being the ideal solution. However, we were unsuccessful in trying to improve upon it. So, despite its shortcomings, this transformation was used in conjunction with all the data analysis.

Through the use of standard statistical tests of significance (the F-test and the T-test), selectively applied, it was concluded that tube position and vibration have no consistent effect on powder emission. Accordingly, these variables were eliminated from further analysis, and the primary effort was concentrated on ascertaining the effects of orifice size and helium pressure.

$$
\text { Analysis of similar data by Pacific Northwest Laboratory }{ }^{(5)}
$$

suggested that powder emission is related to $A \sqrt{P}$, where $A$ is the crosssectional area of the orffice and $P$ is helium pressure. Consequently, we explored the effect of this quantity on the net emission of pultonium oxide powder in the Battelle-Columbus experiments. The first step was to construct a scatter plot showing emission (on transformed scale) versus $A \sqrt{\mathrm{P}}$. On the basis of this plot, the data were separated into groups for further study. The first separation was based upon the value of $A \sqrt{\mathrm{P}}$, and resulted in three experimental regions. Region I consisted of all data for which the value of $A \sqrt{P}$ was less than 2,000. Region II consisted of all data for which the value of $A \sqrt{P}$ was greater than or equal to 2,000 , but smaller than 20,000. Region III consisted of all data for which the value of $\mathrm{A} \sqrt{\mathrm{P}}$ was greater than or equal to 20,000 . The data within each region were further separated into two groups corresponding to Phase I and Phase II of the experimental program. In total, this cross-classification resulted in five groups of orifice data, each of which was analyzed separately.

The data corresponding to Region I and Phase I, as defined above, consists of 46 measurements of $\mathrm{PuO}_{2}$ emissions. These measurements represent six combinations of orifice size and hellum pressure, each combination 
constituting a separate subgroup, as shown in Table 15 in order of increasing values of $A \sqrt{p}$. Summary statistics for each of these subgroups are presented in the table, in both original units and transformed units. These statistics support the assumption of negligible differences among subgroups. Accordingly, all six subgroups were combined to produce the histogram in Figure $21 \mathrm{~A}$ and the overall statistics in Table 15. Taking into account the effect of sampling error and sample size, the histogram has the sort of shape to be expected from a homogeneous set of data. It is reasonable to suppose that it represents a random sample from a unimodal distribution which is slightly skewed toward the right.

The data corresponding to Region I, Phase II, consists of 58 measurements of plutonium oxide emissions. These measurements represent six combinations of orffice size and helium pressure, as shown in Table 16 in order of increasing values of $A \sqrt{\mathrm{P}}$. Summary statistics for each of these subgroups are presented in the table. These statistics support the assumption of negligible differences among subgroups. Accordingly, all six subgroups were combined to produce the histogram in Figure $20 \mathrm{~B}$ and the summary statistics in the last column of Table 16. As before, the histogram has the sort of shape to be expected from a homogeneous set of data.

From visual inspection, it appears that the two histograms in Figures $20 \mathrm{~A}$ and $20 \mathrm{~B}$ represent random samples from the same population. This conclusion is supported by standard parametric tests of statistical significance ${ }^{(a)}$. Accordingly, the two histograms were pooled to obtain the histogram in Figure 20C.

The data corresponding to Region II, Phase I, consists of 35 measurements of plutonium oxide emissions. These measurements represent three combinations of orifice size and helium pressure, as shown in Table 17 in order of increasing values of $A \sqrt{\mathrm{P}}$. These statistics support the assumption of negligible differences among subgroups. Accordingly, all

(a) Student's t-test shows no significant difference between the means of the two histograms, and Fisher's F-test shows no significant difference between the variances. 
TABLE 15. SUMMARY STATISTICS FOR REGION I, PHASE I

\begin{tabular}{|c|c|c|c|c|c|c|c|c|}
\hline \multirow{2}{*}{\multicolumn{2}{|c|}{ Statistics }} & \multicolumn{6}{|c|}{ Subgroup } & \multirow{2}{*}{$\begin{array}{l}\text { Total } \\
\text { Sample }\end{array}$} \\
\hline & & 1 & 2 & 3 & 4 & 5 & 6 & \\
\hline$A \sqrt{\mathrm{P}}$ & & 73 & 294 & 439 & 621 & 1,175 & 1,756 & \\
\hline Diameter, & $\mu \mathrm{m}$ & 5 & 10 & 5 & 5 & 20 & 10 & \\
\hline Pressure, & ps1 & 14 & 14 & 500 & 1,000 & 14 & 500 & \\
\hline \multirow[t]{2}{*}{ Number of } & Runs & 6 & 6 & 6 & 8 & 14 & 6 & 46 \\
\hline & & \multicolumn{5}{|c|}{ or1ginal Scale } & a & \\
\hline Minimum & & -1 & -1 & 1 & -11 & -1 & 4 & -11 \\
\hline Median & & 3 & 3 & 3 & 1 & 8 & 6 & 5 \\
\hline Average & & 4 & 11 & 4 & 1 & 11 & 10 & 7 \\
\hline Maximum & & 13 & 49 & 7 & 12 & 27 & 34 & 49 \\
\hline Standard I & Deviation & 6 & 19 & 2 & 7 & 10 & 11 & 11 \\
\hline \multirow[t]{2}{*}{ Skewness } & & 0.7 & 2.2 & 0.7 & -0.3 & 0.6 & 2.3 & 1.9 \\
\hline & & \multicolumn{6}{|c|}{ Transformed Scale } & \\
\hline Minimum & & 0.94 & 0.98 & 1.06 & 0.00 & 0.95 & 1.14 & 0.00 \\
\hline Median & & 1.12 & 1.10 & 1.11 & 1.06 & 1.26 & 1.21 & 1.16 \\
\hline Average & & 1.13 & 1.22 & 1.13 & 0.94 & 1.28 & 1.27 & 1.17 \\
\hline Maximum & & 1.37 & 1.77 & 1.23 & 1.33 & 1.57 & 1.64 & 1.77 \\
\hline Standard $\mathrm{r}$ & Deviation & 0.18 & 0.30 & 0.07 & 0.42 & 0.21 & 0.19 & 0.27 \\
\hline Skewness & & 0.2 & 1.6 & 0.5 & -1.8 & 0.0 & 2.1 & -1.4 \\
\hline
\end{tabular}




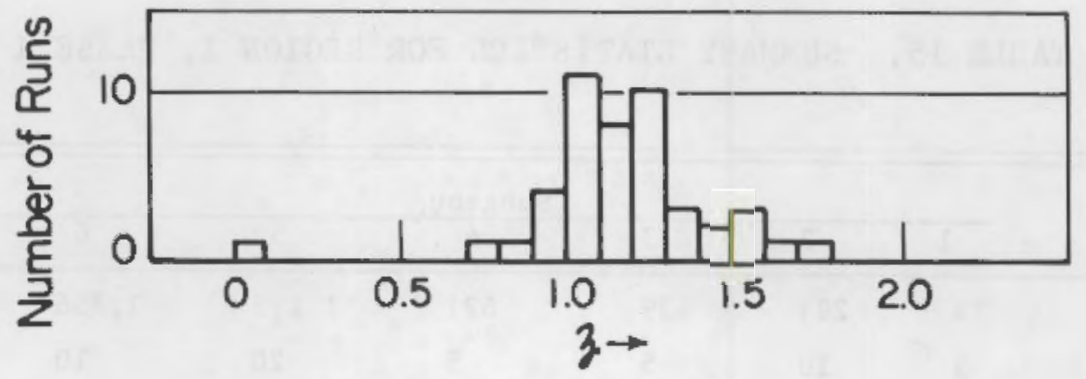

A. Phase I

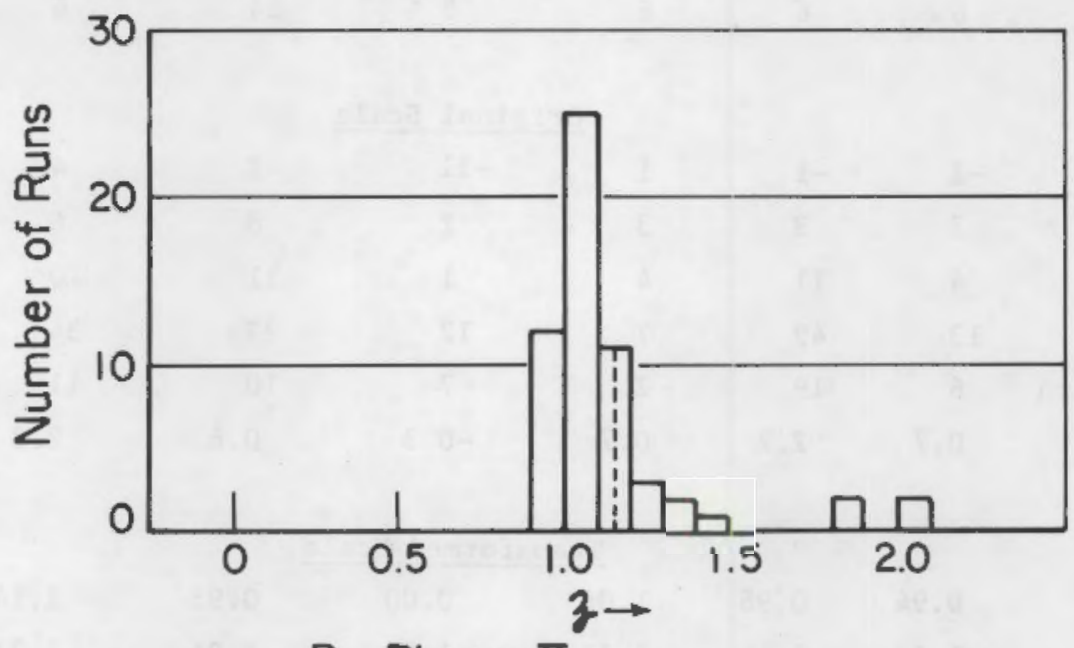

B. Phase II

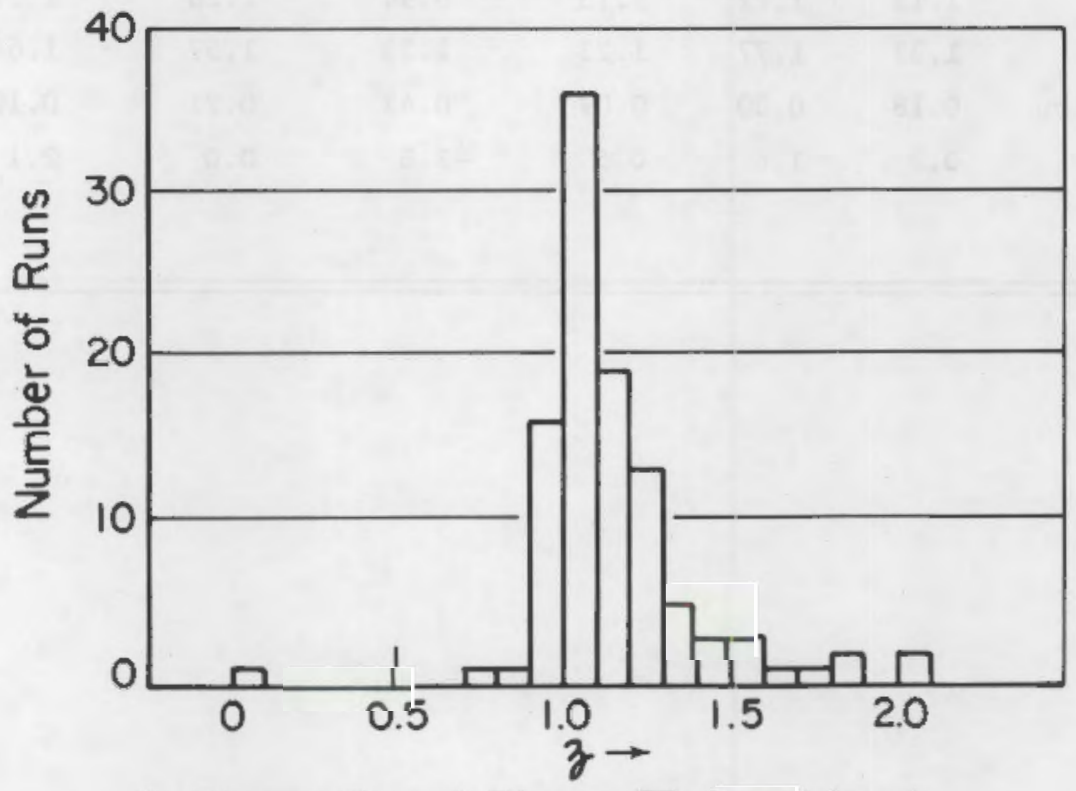

C. Phase I and Phase II Combined

FIGURE 20. DISTRIBUTION OF PLUTONIUM EMISSION (ON TRANSFORMED SCALE, Z) FOR EXPERIMENTAL REGION I $(A \sqrt{\mathrm{P}}<2000)$ 
TABLE 16. SUMMARY STATISTICS FOR REGION I, PHASE II

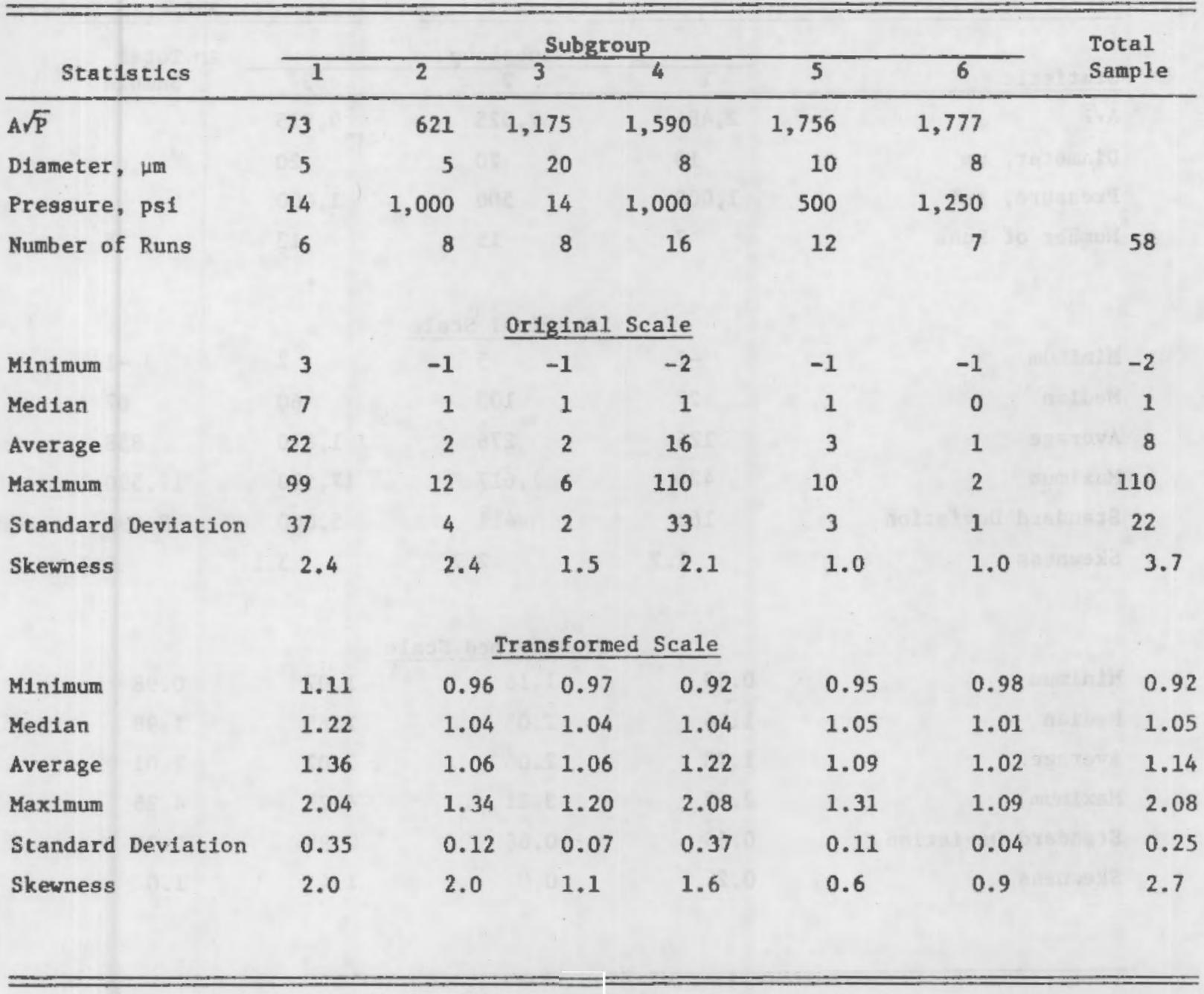


TABLE 17. SUMMARY STATISTICS FOR REGION II, PHASE I

\begin{tabular}{|c|c|c|c|c|}
\hline \multirow{2}{*}{ Statistic } & \multicolumn{3}{|c|}{ Subgroup } & \multirow{2}{*}{$\begin{array}{l}\text { Total } \\
\text { Sample }\end{array}$} \\
\hline & 1 & 2 & 3 & \\
\hline$A \sqrt{\mathrm{P}}$ & 2,484 & 7,025 & 9,935 & \\
\hline Diameter, $\mu \mathrm{m}$ & 10 & 20 & 20 & \\
\hline Pressure, ps1 & 1,000 & 500 & 1,000 & \\
\hline Number of Runs & 7 & 15 & 13 & 35 \\
\hline
\end{tabular}

Minimum

Median

Average

Maximum

Staudard Deviation

Skewness

Minimum
Median
Average
Maximum
Standard Deviation
Skewness

Skewness

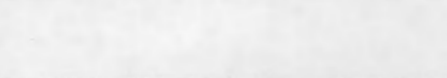

\section{Original Scale}

$\begin{array}{rrrr}-1 & 5 & 2 & -1 \\ 27 & 103 & 60 & 87 \\ 126 & 276 & 1,870 & 838 \\ 420 & 1,617 & 17,590 & 17,590 \\ 163 & 411 & 5,000 & 3,090 \\ 1.2 & 2.7 & 3.1 & 5.1\end{array}$

Transformed Scale

$\begin{array}{llll}0.98 & 1.16 & 1.07 & 0.98 \\ 1.56 & 2.05 & 1.85 & 1.98 \\ 1.78 & 2.06 & 2.07 & 2.01 \\ 2.63 & 3.21 & 4.25 & 4.25 \\ 0.64 & 0.66 & 0.95 & 0.76 \\ 0.2 & 0.0 & 1.4 & 1.0\end{array}$


three subgroups were combined to produce the histogram in Figure $21 \mathrm{~A}$ and the summary statistics in the last column of Table 17. This histogram contrasts sharply with the histogram for Region I, but the difference may simply be due to the erratic nature of small samples.

The data corresponding to Region II, Phase II, consists of 46 measurements of plutonium oxide emissions. These measurements represent two combinations of orifice size and helium pressure, as shown in Table 18 in order of increasing values of $A \sqrt{\mathrm{P}}$. These statistics support the assumption of negligible differences among subgroups. Accordingly, both subgroups were combined to produce the histogram in Figure $22 \mathrm{~B}$ and the summary statistics in the last column of Table 18. This histogram is fairly conventional in shape.

From visual inspection, it appears that the two histograms in Figures $21 \mathrm{~A}$ and $21 \mathrm{~B}$ represent random samples from totally different populations. This conclusion is supported by a standard nonparametric test of statistical significance ${ }^{(b)}$. Nevertheless, the two histograms were pooled to obtain the histogram in Figure 21C, in order to visualize the consequences of a different approach. From the standpoint of predicting future performance, it makes sense to combine Phase I and Phase II data, even though they don't agree. What are the alternatives? It is difficult to come up with a sensible rationale for two separate populations. Even if such a rationale could be found, it would involve the risk of undẹrestimating the experimental error.

The data corresponding to Region III, Phase II, consists of 65 measurements of plutonium oxide emissions (Region III is devoid of any Phase I data). These measurements represent three combinations of orifice size and helium pressure, as shown in Table 19 in order of increasing values of $A \sqrt{P}$. These statistics support the assumption of negligible differences among subgroups. Accordingly, all three subgroups were combined to produce the summary statistics in the last column of Table 19.

(b) The Wilcoxon-Mann-Whitney test. 


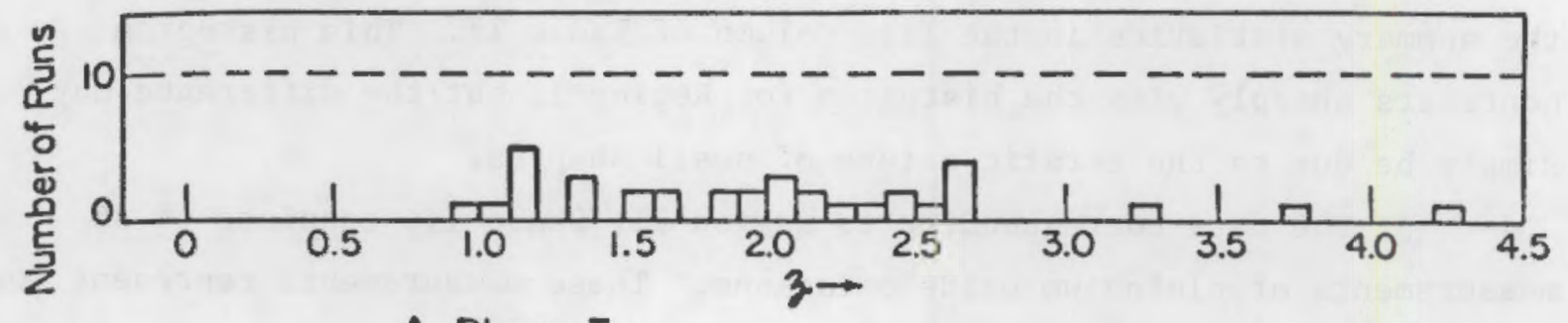

A. Phase I

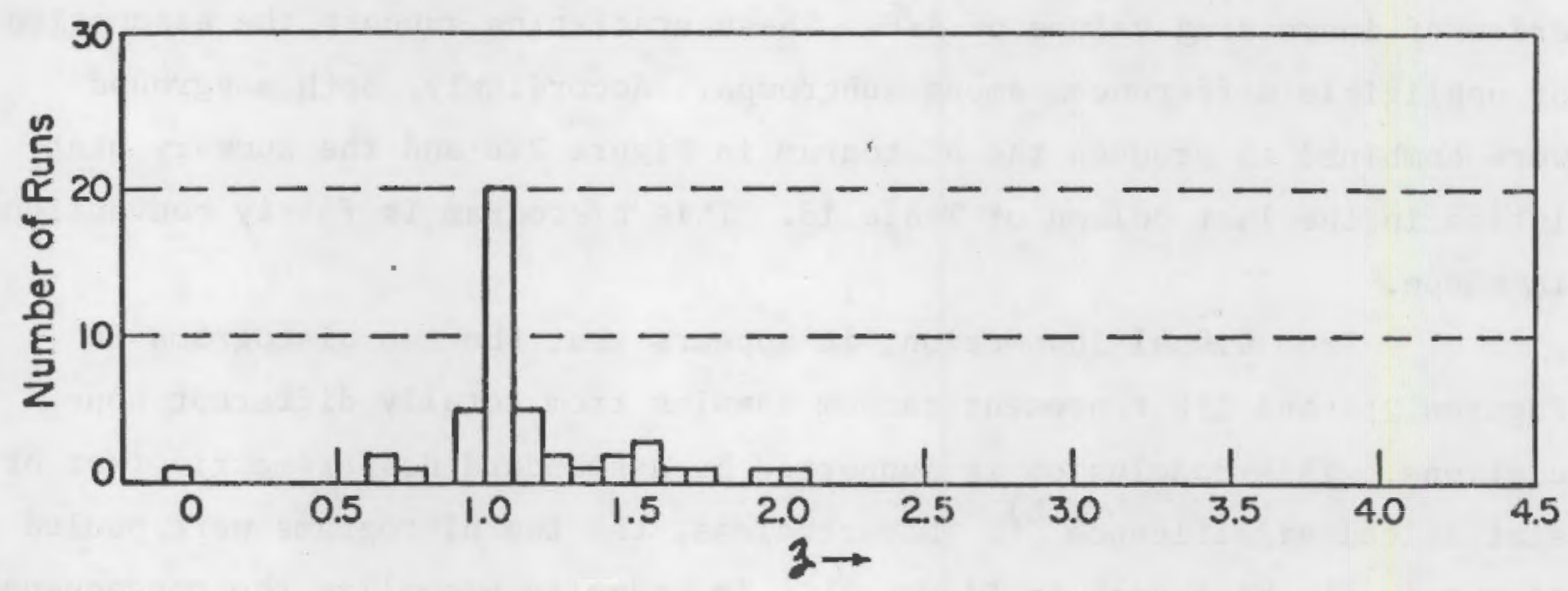

B. Phase II

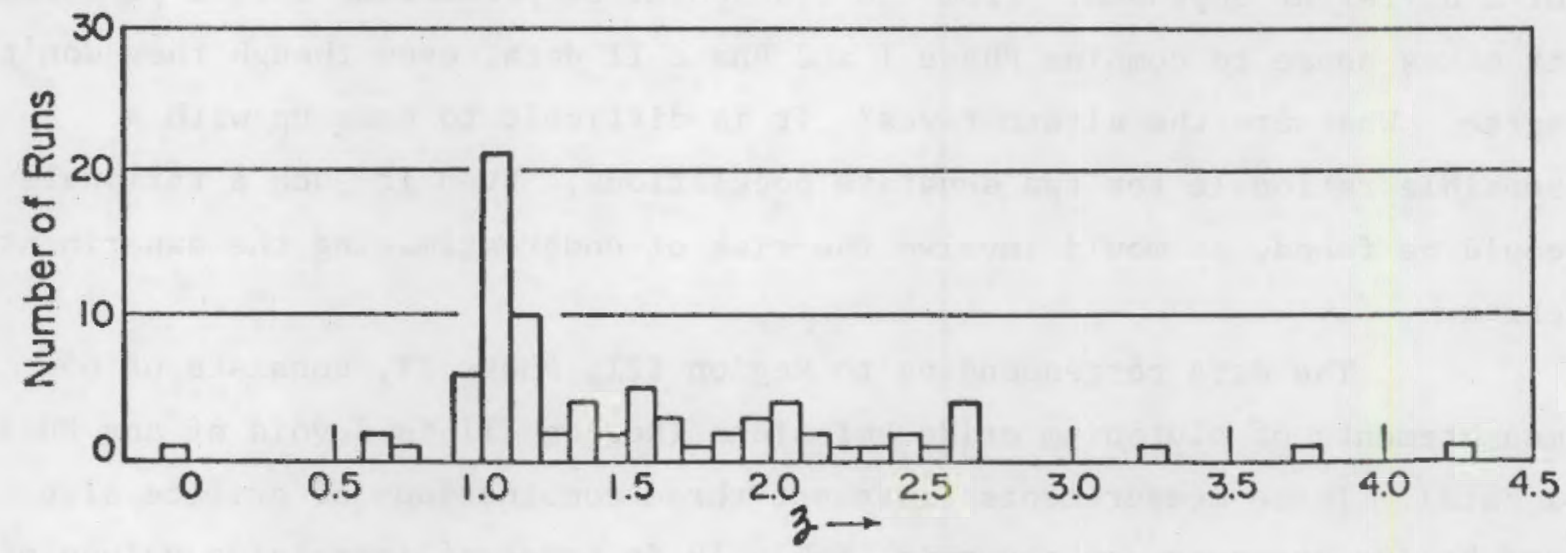

C. Phase I and Phase II Combined

FIGURE 21. DISTRIBUTION OF PLUTONIUM EMISSION (ON TRANSFORMED SCALE,Z) FOR EXPERIMENTAL REGION II $(2000 \leq \mathrm{A} \sqrt{\mathrm{P}}<20,000)$ 
TABLE 18. SUMMARY STATISTICS FOR REGION II, PHASE II

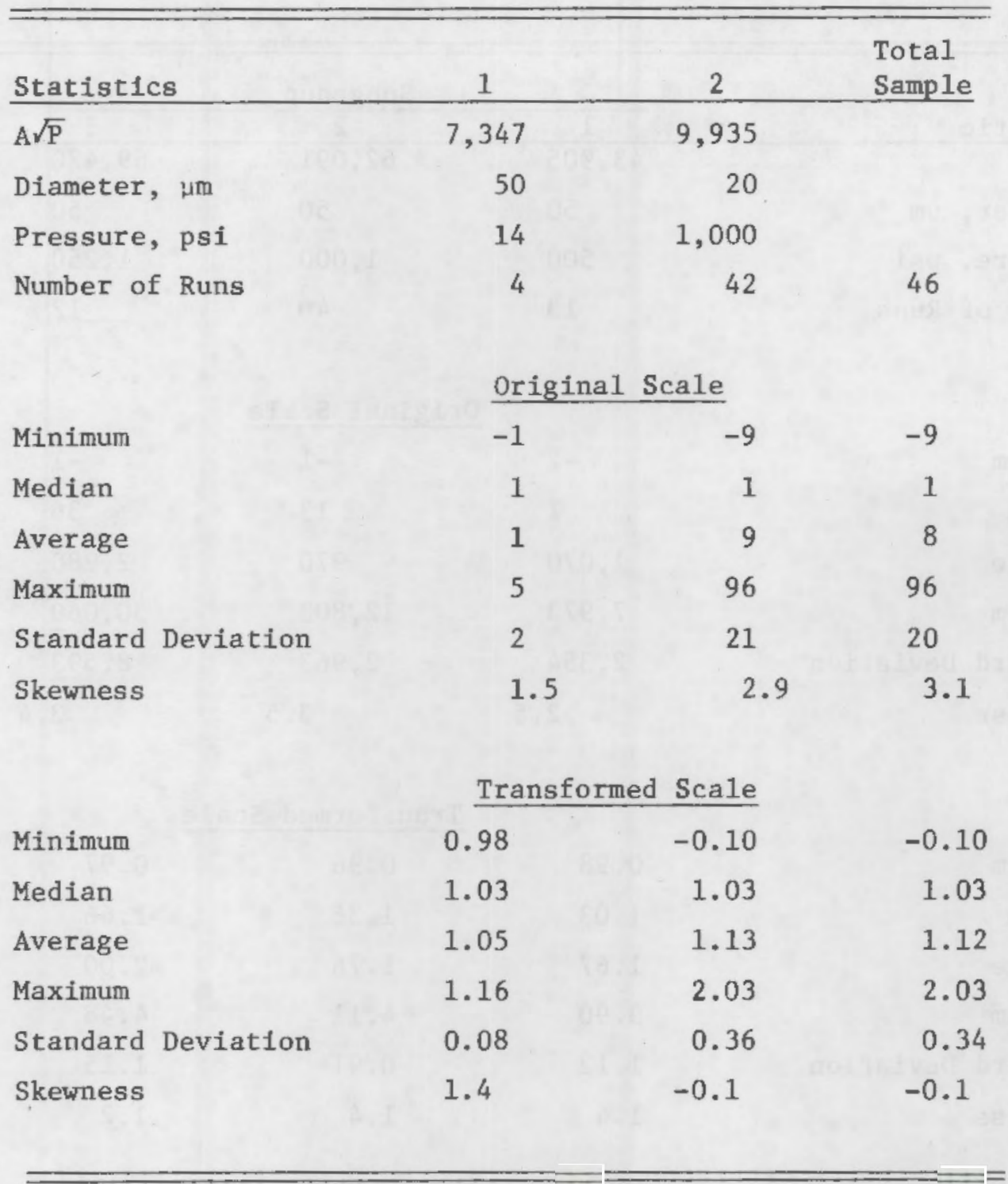


TABLE 19. SUMMARY STATISTICS FOR REGION III, PHASE II

\begin{tabular}{|c|c|c|c|c|}
\hline \multirow[b]{2}{*}{ Statist1c } & \multicolumn{3}{|c|}{ Subgroup } & \multirow{2}{*}{$\begin{array}{l}\text { Total } \\
\text { Sample }\end{array}$} \\
\hline & 1 & 2 & 3 & \\
\hline $\mathrm{A} \sqrt{\mathrm{P}}$ & 43,905 & 62,091 & 69,420 & \\
\hline Diameter, $\mu \mathrm{m}$ & 50 & 50 & 50 & \\
\hline Pressure, psi & 500 & 1,000 & 1,250 & \\
\hline Number of Runs & 13 & 40 & 12 & 65 \\
\hline
\end{tabular}

\begin{tabular}{lrrrr} 
& \multicolumn{3}{c}{ Original Scale } & \\
Minimum & -2 & -1 & -1 & -2 \\
Median & 1 & 13 & 36 & 13 \\
Average & 1,070 & 970 & 2,980 & 1,361 \\
Maximum & 7,973 & 12,800 & 30,060 & 30,060 \\
Standard Deviation & 2,354 & 2,963 & 8,593 & 4,437 \\
Skewness & 2.5 & 3.5 & 3.4 & 5.0
\end{tabular}

Transformed Scale

$\begin{array}{lllll}\text { Minimum } & 0.98 & 0.96 & 0.97 & 0.93 \\ \text { Median } & 1.03 & 1.36 & 1.66 & 1.36 \\ \text { Average } & 1.67 & 1.76 & 2.00 & 1.79 \\ \text { Maximum } & 3.90 & 4.11 & 4.48 & 4.48 \\ \text { Standard Deviation } & 1.12 & 0.91 & 1.15 & 0.99 \\ \text { Skewness } & 1.4 & 1.4 & 1.2 & 1.3\end{array}$


Figure 22 summarizes the results of the analysis of orifice data. The histogram in Figure $22 \mathrm{~A}$ is taken from Figure $20 \mathrm{C}$, and the histogram in Figure 22B is taken from Figure 21C. The histogram in Figure 22C represents the combined data (65 runs) for Region III, Phase II (there is no data for Region III, Phase I). Summary statistics for these three histograms are presented in Table 20. It is evident that the center of the distribution (as measured by the median and the average), the variation due to experimental error (as measured by the standard deviation) and the skewness all increase as the value of $A \sqrt{P}$ increases from one region to the next. This is in approximate comformance with results obtained by Pacific Northwest Laboratory ${ }^{(5)}$ analogous data. Although a trend is indicated by the results shown in Figure 22 , the form of the relationship cannot be determined from the present data bank.

On the basis of the above observations and analyses, three conclusions may be drawn:

1. There is no observable parametric relationship between the emission of $\mathrm{PuO}_{2}$ powder and any single parameter (leak size/ type, position, agitation, pressure, run time).

2. The only parametric dependence suggested by the data is a relationship between the logarithmic transformation of the data and $\log _{10} A \sqrt{\mathrm{P}}$. This functional relationship is weak and the data are not sufficient to allow a more detailed function to be determined.

3. The lack of a dependence on the run time indicates that the majority of $\mathrm{PuO}_{2}$ powder emitted during a run is emitted during the initial pressurization. 


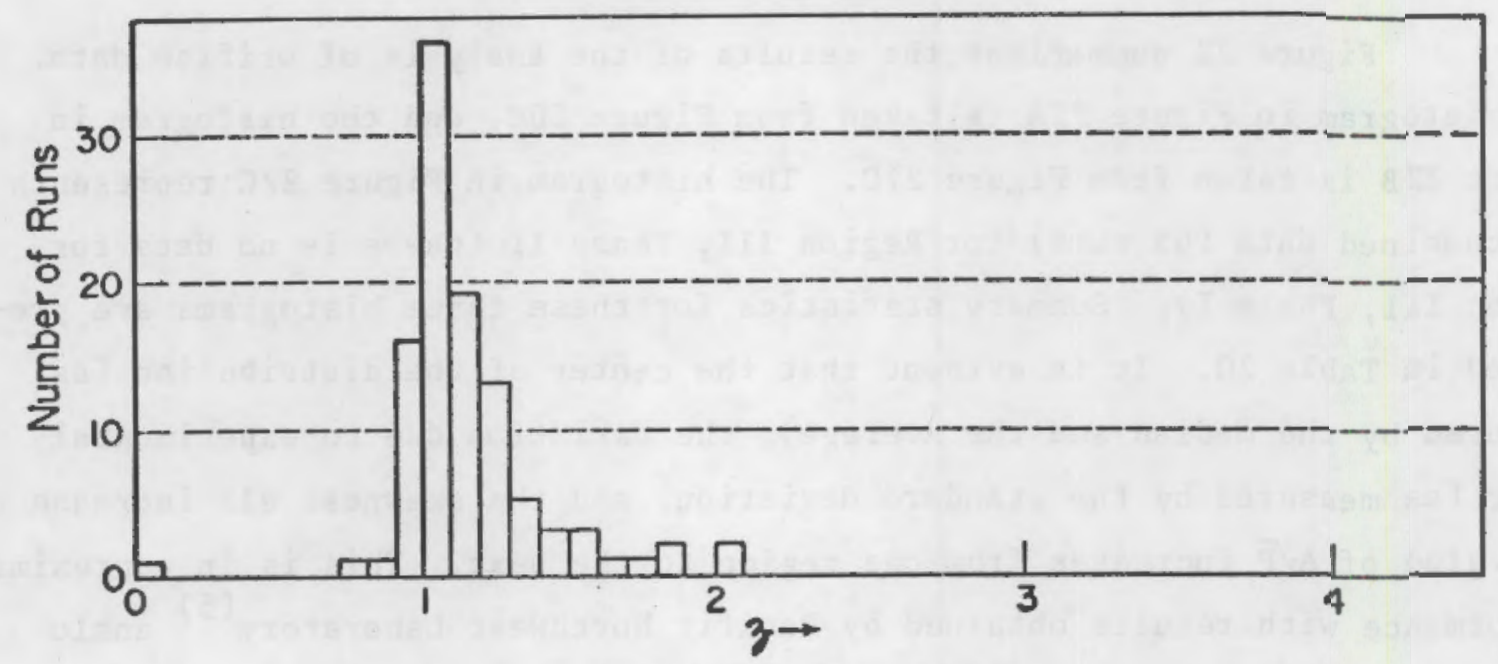

A. Region I $(A \sqrt{P}<2000$

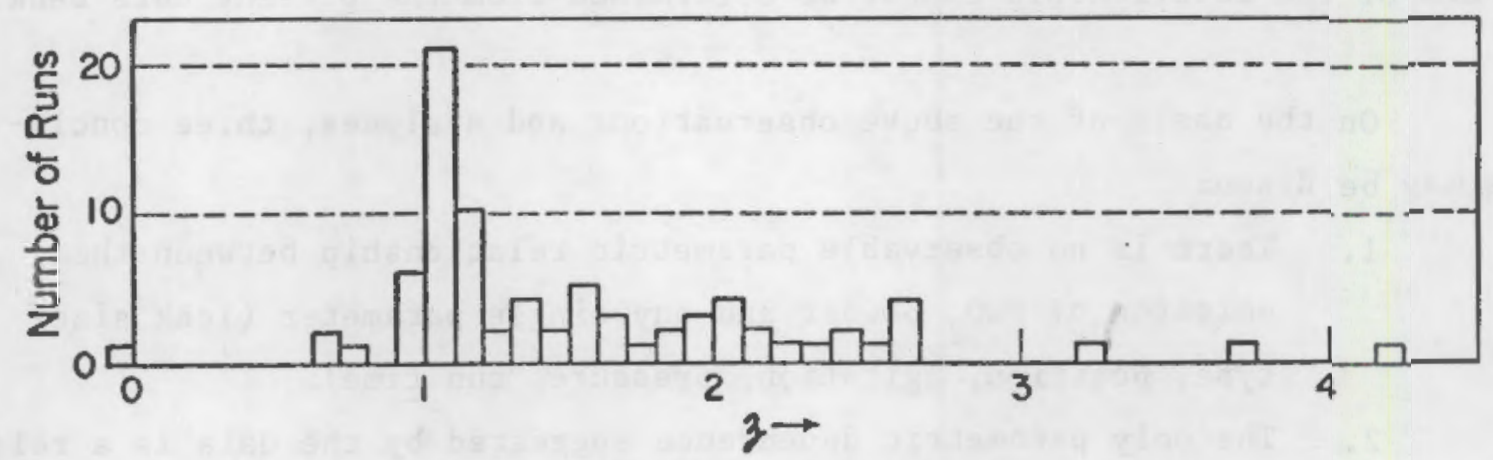

B. Region II $(2000 \leq A \sqrt{P}<20,000$

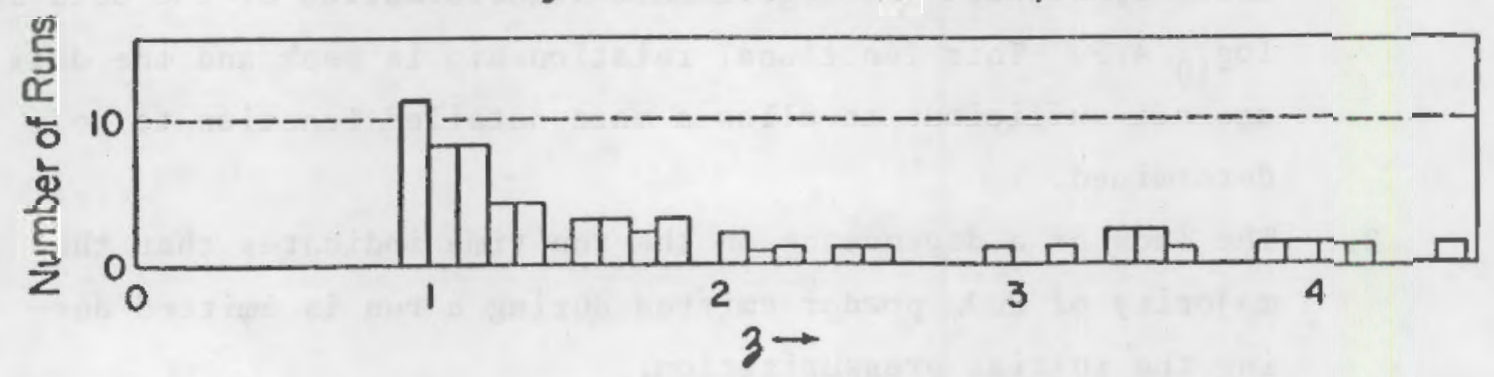

C. Region III $(A \sqrt{P} \geq 20,000$

FIGURE 22. DISTRIBUTION OF PLUTONIUM EMISSION (ON TRANSFORMED SCALE, Z) FOR THREE EXPERIMENTAL REGIONS 
TABLE 20. SUMMARY STATISTICS FOR REGIONS I, II, AND III

\begin{tabular}{|c|c|c|c|}
\hline \multirow[b]{2}{*}{ Statistic } & \multicolumn{3}{|c|}{ Region } \\
\hline & I & II & III \\
\hline \multirow[t]{2}{*}{ Number of Runs } & 104 & 81 & 65 \\
\hline & \multicolumn{3}{|c|}{ Original Scale } \\
\hline Minimum & -11 & -9 & 645 \\
\hline Median & 2 & 6 & 13 \\
\hline Average & 8 & 367 & 1,361 \\
\hline Maximum & 110 & 17,590 & 30,060 \\
\hline Standard Deviation & 18 & 2,057 & 4,437 \\
\hline \multirow[t]{2}{*}{ Skewness } & 4.0 & 7.8 & 5.0 \\
\hline & \multicolumn{3}{|c|}{ Transformed Scale } \\
\hline Minimum & 0.00 & -0.10 & 0.93 \\
\hline Median & 1.08 & 1.20 & 1.36 \\
\hline Average & 1.15 & 1.51 & 1.79 \\
\hline Maximum & 2.08 & 4.25 & 4.48 \\
\hline Standard Deviation & 0.26 & 0.71 & 0.99 \\
\hline Skewness & 0.7 & 1.4 & 1.3 \\
\hline
\end{tabular}




\section{$\stackrel{P u O}{2}_{2}$ Mass to Helium Flow Correlation}

A correlation between the mass of $\mathrm{PuO}_{2}$ emitted during an experiment and the total helium flow could be useful in determining leak specifications for shipping containers. Further, it was agreed upon with Pacific Northwest Laboratory personnel that all the topical reports associated with the plutonium oxide leak rate experiments would present the data in terms of totsl helium flow and $\mathrm{PuO}_{2}$ emitted. To this end, columns six, eight and nine are included in the tables of Appendix B. These columns are, respectively, total helium flow in scc, net total mass of plutonia detected, and the plutonia mass to

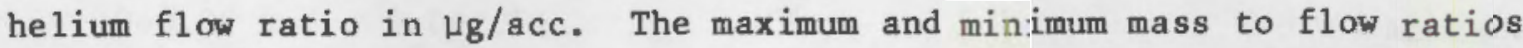
observed for the pressurized orifice experiments are presented in Table 21. As inspection of the table reveals, the maximum value observed was $5 \mathrm{x}$ $10^{-3} \mathrm{\mu g} / \mathrm{cc}$ and the minimum observed value $3 \times 10^{-8} \mathrm{\mu g} / \mathrm{cc}$. 
TABLE 21. PLUTONIA MASS/HELIUM FLOW CORRELATION $\left(\mu \mathrm{g} / \mathrm{cm}^{3}\right)$ (PRESSURIZED LEAK TUBE)

\begin{tabular}{ccc}
\hline $\begin{array}{c}\text { Orifice } \\
\text { Size }(\mu \mathrm{m})\end{array}$ & \multicolumn{2}{c}{ Mass/Flow } \\
\hline 5 & $9 \times 10^{-8}$ & $2 \times 10^{-5}$ \\
8 & $3 \times 10^{-8}$ & $4 \times 10^{-5}$ \\
10 & $1 \times 10^{-7}$ & $2 \times 10^{-4}$ \\
20 & $3 \times 10^{-8}$ & $4 \times 10^{-3}$ \\
50 & $1 \times 10^{-7}$ & $5 \times 10^{-3}$ \\
\hline
\end{tabular}

Only positive non-zero value included 



\section{REFERENCES}

(1) ANSI-N14-5, Leakage Tests on Packages for Shipment of Radioactive Materials, November, 1974.

(2) Regulatory Guide 7.4, Leakage Tests on Packages for Shipment of Radioactive Materials, U.S. Nuclear Regulatory Commission, June, 1975.

(3) IAEA Safety Series No. 6, Regulations for the Safe Transport of Radioactive Materials, Table VII, $1973 \mathrm{ed}$.

(4) L. C. Schwendiman, Supporting Information for the Estimation of Plutonium Oxide Leak Rates Through Very Small Apertures, BNWL-2198, Pacific Northwest Laboratory, 1977.

(5) Sutter, S. L., J. W. Johnson, J. Mishima, P. C. Owzarski, L. C. Schwendiman and G. B. Long, Depleted Uranium Dioxide Powder Flow Through Very Small Openings, NUREG/CR-1099, PNL-3177, Pacific Northwest Laboratory, Richland, WA, 1980.*

*Ava1lable for purchase from the NRC/GPO Sales Program, U.S. Nuclear Regulatory Commission, Washington, DC 20555, and the National Technical Information Service, Springfield, VA 22161. 

APPENDIX A

LASER OPTICAL PARTICLE MONITORING SYSTEM (LOPMS) 


\section{APPENDIX A}

\section{LASER OPTICAL PARTICLE MONITORING SYSTEM (LOPMS)}

A real-time, continuous monitoring instrument would be highly useful in characterizing particulate leaks from small cracks. The number of particles emitted from a given leak, the particulate leak rate, the change in the leak rate with time, and the particle size distribution are the desired data. To obtain this information, the development of a laser optical particle monitoring system was undertaken.

The system was designed to detect light scattered by leak particles as they pass through a uniform plane of laser light intercepting the leak path. A schematic representation of the system is shown in Figure Al. As a particle passes through the plane of laser light it scatters light in all directions. The light scattered at a fixed solid angle in the forward direction is collected by a fiber optics bundle and transmitted to a photomultiplier tube (PMT). The associated voltage pulse generated by the PMT is classified according to its magnitude and stored using a multichannel analyzer (MCA). In principle, each detected light pulse corresponds to a leaked particle and the magnitude of the voltage pulse is related to the particle size. By monitoring the counts with time, the total number of particles leaked, the leak rate, and the change in leak rate are measured. Also obtained are the number of counts in each of several intensity classifications, thus indicating a relative particle size distribution.

\section{Experimental}

A series of particle leak rate and size distribution experiments were conducted using a leak tube having a 50-um orifice (Figure A2). Helium gas, at a constant pressure of 500 psig, was supplied to the leak tube during testing. The leak tube was oriented in the upright position, 1.e., emftting particles in the upward direction. $\mathrm{ThO}_{2}$ powder was used as a simulant for $\mathrm{PuO}_{2}$ in the experiments. 


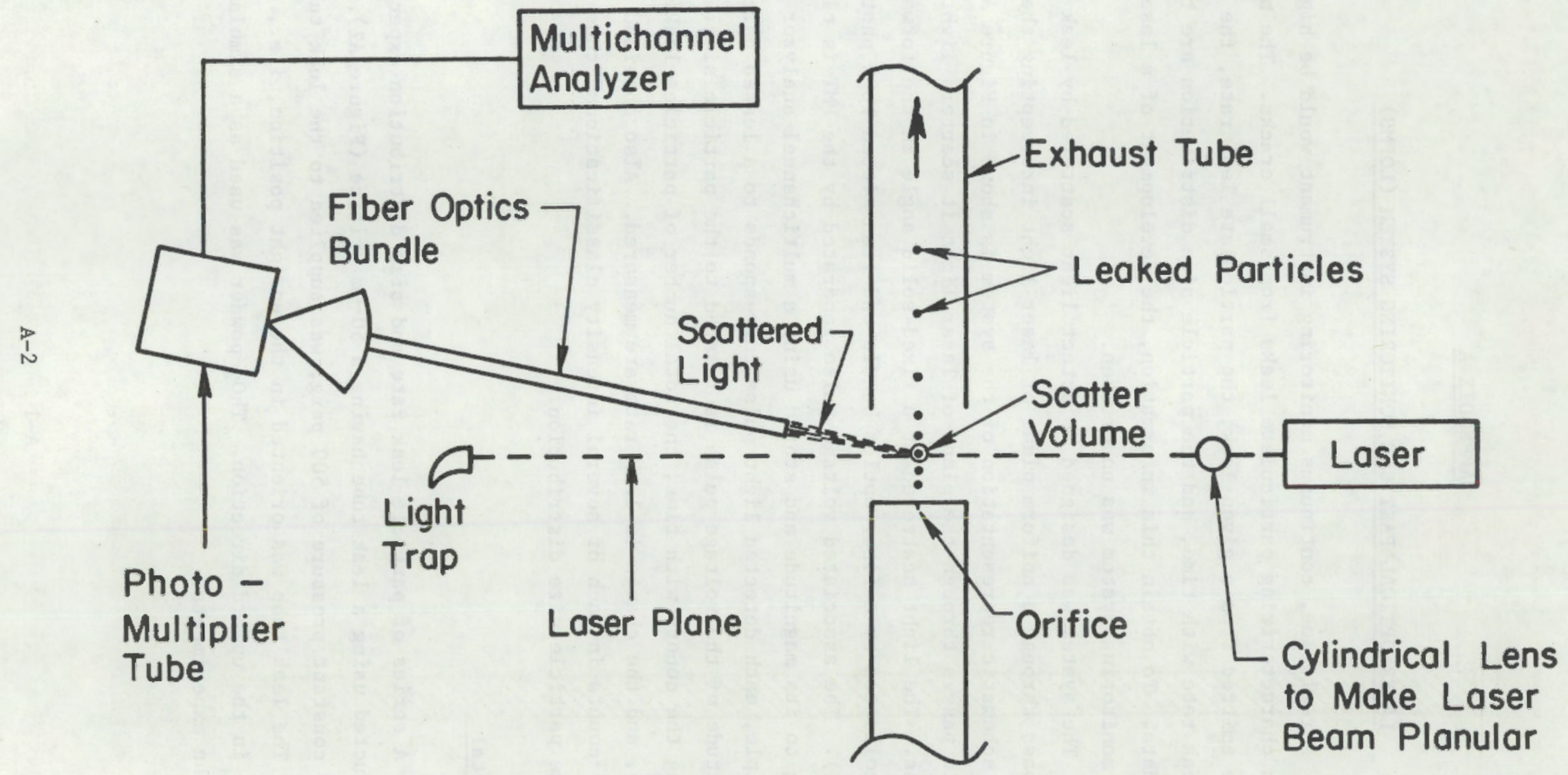


$\mathrm{He}+\mathrm{ThO}_{2}$ Particles

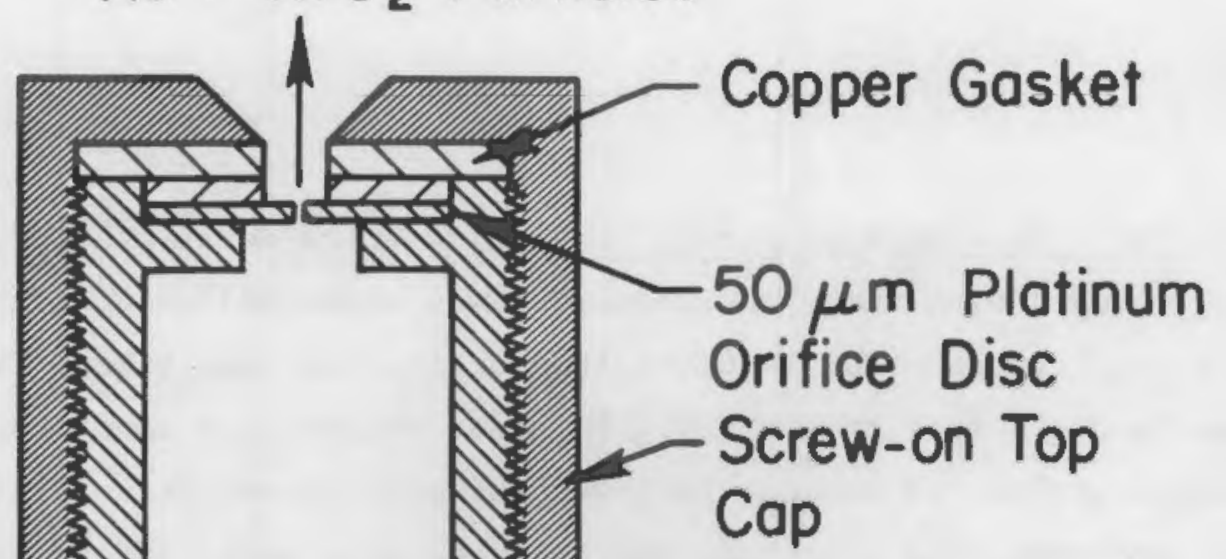

Stainless Steel
Body

Stainless Steel
Body

v

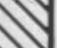

-

(

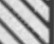

(

.

N

N

N

H

Filter $\quad \mathrm{SHe}$

Screw-on

Bottom Cap

FIGURE A2. LEAK TUBE USED FOR THE PLUTONIA EXPERIMENTS 
Particle Leak Rate Versus Time. To determine if the $\mathrm{ThO}_{2}$ particle leak rate changed with time, an experiment was conducted using a high-speed recording oscillograph (RO) to chart the particulate leak rate. The RO was connected to the output of the PMT. In order to simulate conditions in which a shipping container of nuclear fuel was suddenly jarred or tumbled in a transportation accident, the leak tube was agitated vigorously before being connected to the helium line. Presumably, agitation would distribute $\mathrm{ThO}_{2}$ over the inside wall of the leak tube and suspend some of the finer particles. The leak tube was pressurized to 500 psig with helium and data were taken using the RO over a period of 11 min, cycling the RO: on for 1 min, off for 1 min.

Figures A3a through $f$ show typical section of the Ro chart for the first, third, fifth, seventh, ninth, and eleventh min of the run. The particle leak rate was greatest during the first min, decayed to $\sim 10$ to 20 percent of the initial rate during the third min, and finally decayed to an approximately constant rate of 1 to 5 percent of the initial rate for the fifth through eleventh minutes. Background due to room aerosol was determined to be $\sim 10$ to 20 percent of the final, constant particle emission rate. Each peak was assumed to be a light pulse generated by a single $\mathrm{ThO}_{2}$ particle traversing the laser plane, but no efforts was made to determine absolute particle count rate or detection efficiency. It is interesting to note that from the fifth minute until the end of the run, particle emission appears somewhat periodic rather than continuous. Such periodic emission may have been caused by repeated plugging and unplugging of the orifice or by particles merely being periodically swept off the inside wall of the leak tube. Additional study is needed to determine the mechanism of the apparent periodic emission.

\section{$\underline{\text { Particle Size Distribution }}$}

Calibration of MCA. A series of experiments were conducted in an effort to determine the particle size distribution of $\mathrm{ThO}_{2}$ particles being emitted from the 50- $\mathrm{m}$ orifice at a constant pressure of 500-psig helium. Scattered light pulses, converted to voltage pulses by the PMT, were classified in the MCA according to pulse height. 


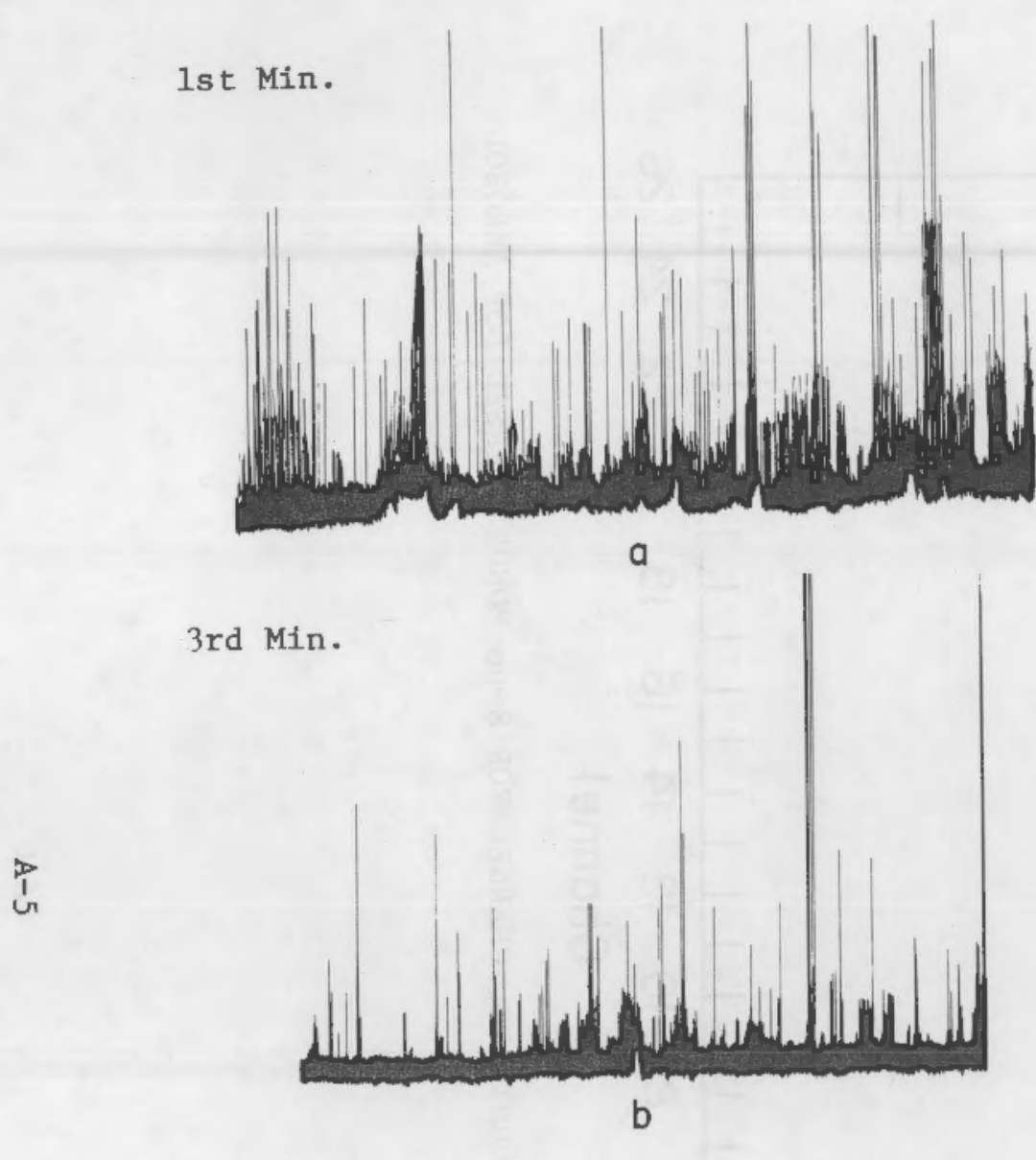

5 th Min.

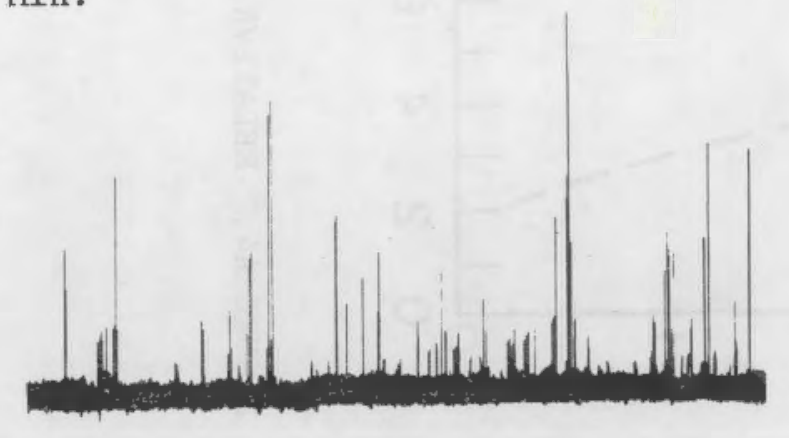

C

7th Min.

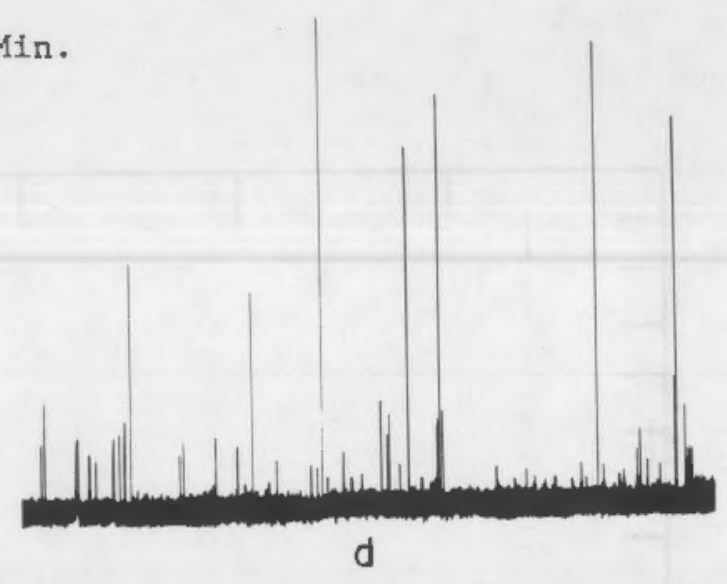

9 th Min.

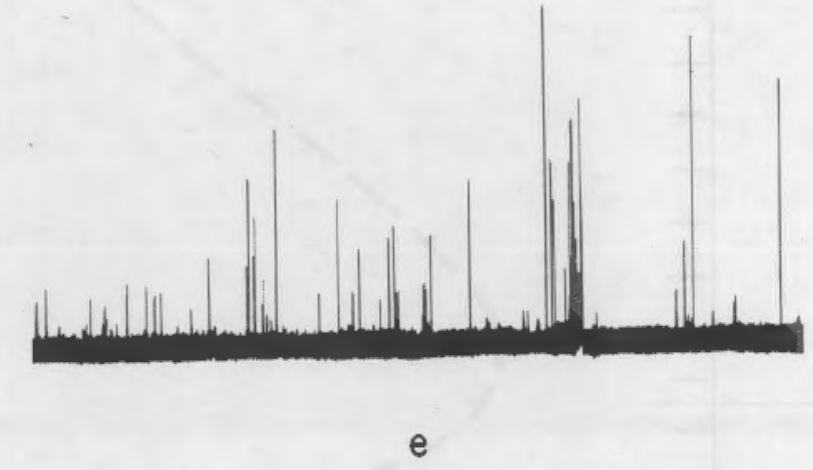

11th Min.

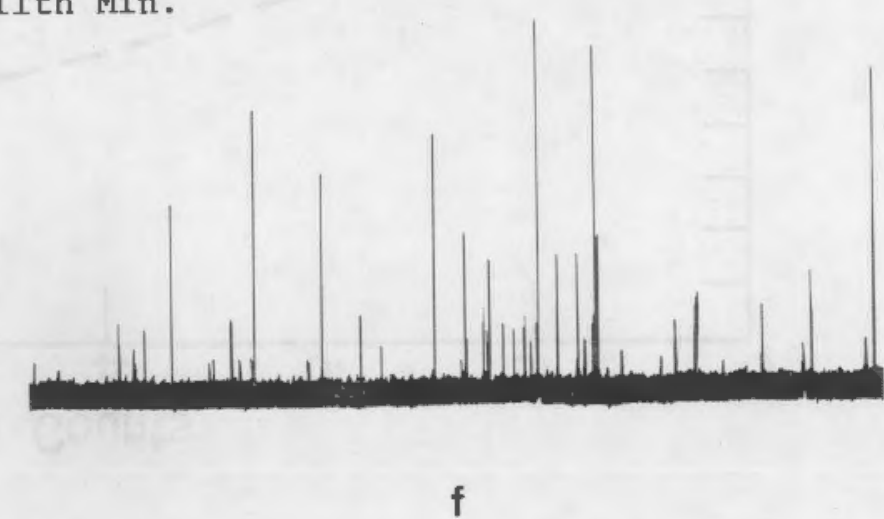

FIGURE A3. RECORDING OSCILLOSCOPE TRACE OF PARTICLE EMISSION RATE 


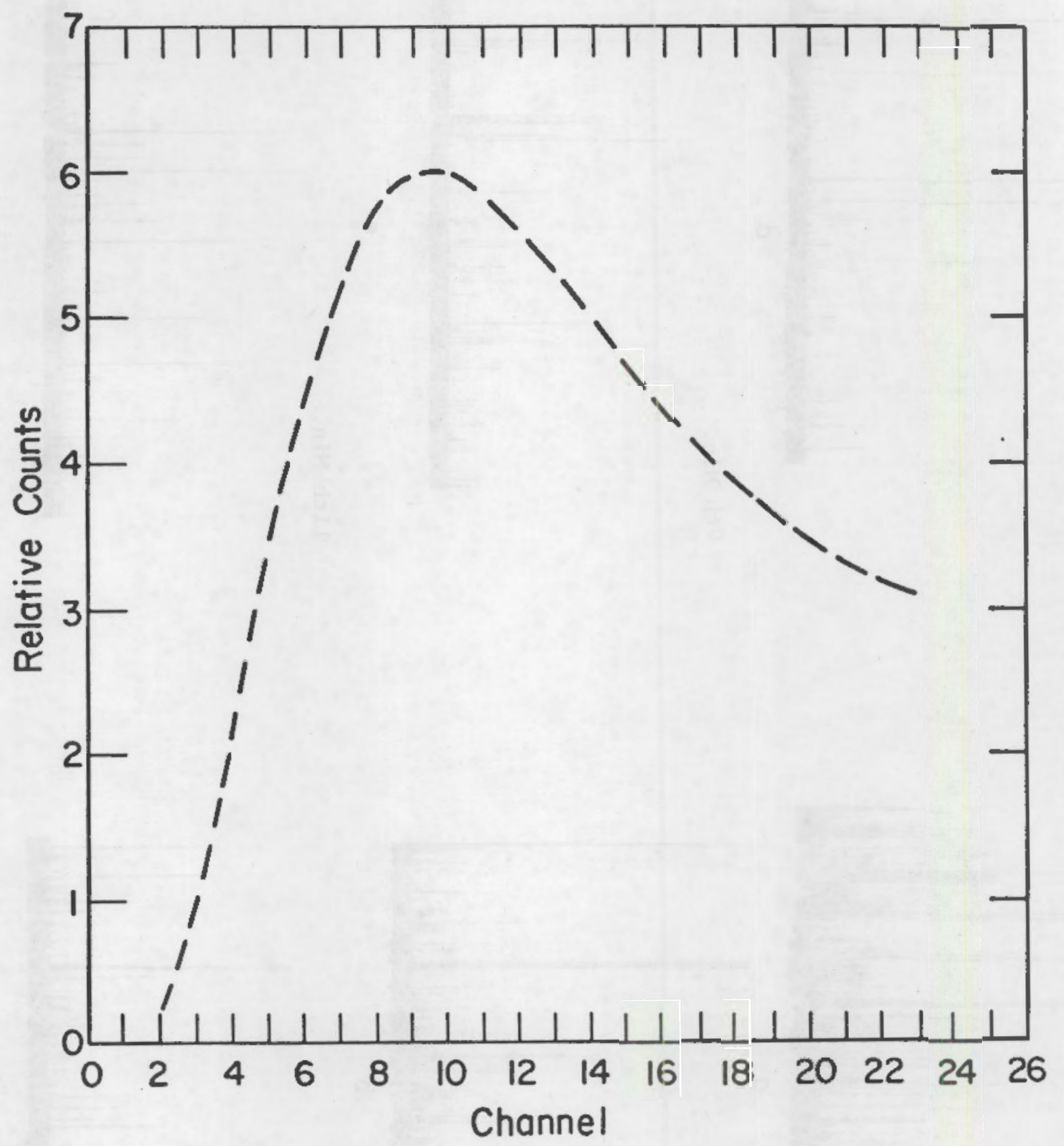

FIGURE A4. RELATIVE COUNTS VS. CHANNEL FOR 8- $4 \mathrm{~m}$ MONODISPERSED DOP AEROSOL 


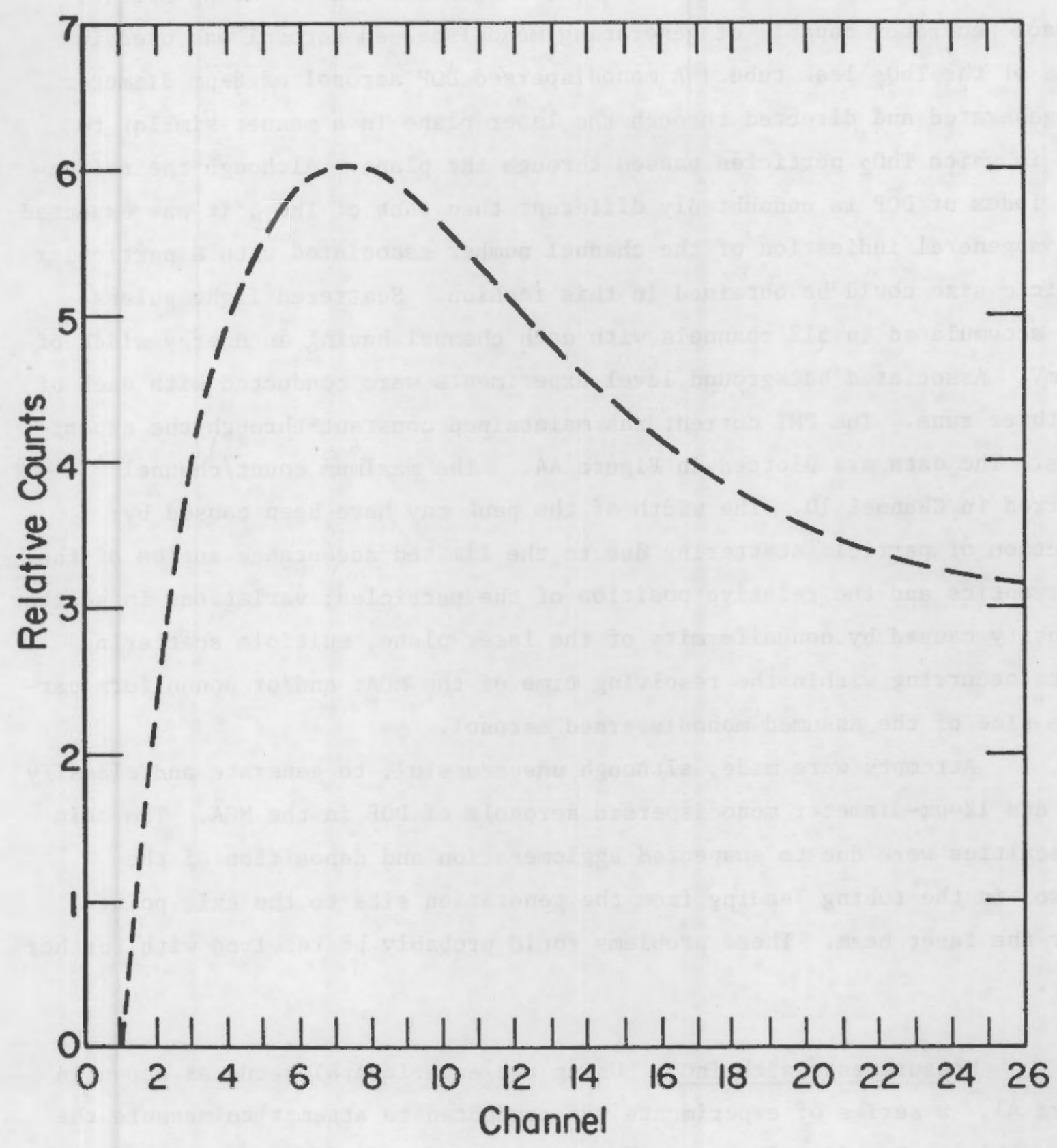

FIGURE A5. RELATIVE COUNTS VS. CHANNEL FOR ThO 2 TEST AEROSOL 
In order to calibrate the MCA, a Berglund-Liu vibrating orifice aerosol generator capable of generating monodispersed aerosol was used in place of the $\mathrm{ThO}_{2}$ leak tube. A monodispersed DOP aerosol of $8-\mu \mathrm{m}$ diameter was generated and directed through the laser plane in a manner similar to that in which $\mathrm{ThO}_{2}$ particles passed through the plane. Although the refractive index of DOP is undoubtedly different than that of $\mathrm{ThO}_{2}$, it was expected that a general indication of the channel number associated with a particular particle size could be obtained in this fashion. Scattered light pulses were accumulated in 512 channels with each channel having an energy width of $\sim 15 \mathrm{mV}$. Associated background level experiments were conducted with each of the three runs. The PMT current was maintained constant through the experiments. The data are plotted in Figure A4. The maximum count/channel occurred in Channel 10. The width of the peak may have been caused by: detection of particle scattering due to the limited acceptance angles of the fiber optics and the relative position of the particles; variations in scatter intensity caused by nonuniformity of the laser plane, multiple scattering events occurring within the resolving time of the MCA; and/or nonuniform particle size of the assumed monodispersed aerosol.

Attempts were made, although unsuccessful, to generate and classify 4- $\mu \mathrm{m}$ and 12- $\mu \mathrm{m}$-diameter monodispersed aerosols of DOP in the MCA. The main difficulties were due to suspected agglomeration and deposition of the aerosol in the tubing leading from the generation site to the exit point under the laser beam. These problems could probably be resolved with further study.

Measurements with Th02. Using the experimental setup as shown in Figure Al, a series of experiments was conducted to attempt to measure the $\mathrm{ThO}_{2}$ particle size distribution. Three runs were made of approximately 3-1/2 minutes' duration each, at 500-psig helium. Background measurements were taken before each run. Figure A5 shows the average relative counts/ channel for $\mathrm{ThO}_{2}$. The maximum count/channel occurred in Channel 7 . A mean particle size of 7-um equivalent diameter had previously been determined by L. Miga of Battelle Columbus Laboratories (December 18, 1976) for the $\mathrm{ThO}_{2}$. Assuming that $\mathrm{DOE}$ and $\mathrm{ThO}_{2}$ scatter light by a similar mechanism, the maximum 
count/channel for $\mathrm{ThO}_{2}$ should occur in a channel number $<10$ as is the case. The overall similarity between the DOP peak spread and the $\mathrm{ThO}_{2}$ peak spread, however, indicate that the attempted particle size distribution measurements were not successful with the present apparatus. Assuming the DOP is truly monodispersed, its peak shape should be considerably different from the peak shape of a material such as $\mathrm{ThO}_{2}$ which is composed of particles ranging from $\sim 0.2 \mathrm{\mu m}$ to $\sim 20 \mathrm{um}$. Additional refinement of the apparatus is required before useful particle size distribution data can be obtained.

\section{Discussion}

The experiments have shown the usefulness of the LOPMS as a monitoring tool to detect relative particulate leak rates. Thus, this technique, when used to monitor a particulate leak rate under given conditions, can measure general trends such as plugging and unplugging of the leak crack. Furthermore, relative amounts of particulate leaked for different conditions may be measured and comparisons drawn using this technique.

In order to use the LOPMS as a quantitative counting tool, a particulate detection efficiency calibration would be necessary. There are no assurances that every leaked particle crossed the laser plane nor that all which did resulted in detectable scattered light. Inherent in the system is a limited "probe volume" from which the scattered light can be detected. This limitation is due to the limited solid angle of the fiber bundle. Also, no correction for the effect of multiple scatters (more than one particle in the probe volume at one time) was made. This calibration could be accomplished by comparing simultaneously LOPMS particle counts with that of another calibrated counting instrument such as a commercially available optical particle counter. Such a calibration was not conducted during the current program.

The size distribution measurement experiments demonstrated the limited use of the LOPMS as a sizing instrument. The results show the need for further system development to reduce and define the peak width. To relate such measurements to an absolute size distribution would require 
calibration by another sizing technique such as a cascade impactor. Even if such a calibration were made there are several problems associated with the LOPMS technique for sizing. The relation between particle size and relative scattering intensity must be known. Such a relationship is dependent in part on refractive index, thus the calibration aerosol must have the same or relatable refractive index as the test aerosol. Furthermore, once a calibration is made, care must be taken to maintain the identical probe volume/fiber detecting head configuration. These considerations made the LOPMS a tedious system to operate for size distribution measurement. There are also problems in interpreting intensity (and thus size) measurements due to multiple simultaneous scattering incidents, partial scattering incidents (only part of a particle passing through the probe volume), and nonuniformity of the laser plane.

\section{Conclusions}

- The laser system described can monitor in real time the relative amounts of particles leaked and trends in particle leak rate.

- The technical feasibility of the LOPMS as a counting instrument has been demonstrated; however, further experimentation is needed before this use can be demonstrated quantitatively.

- Such a system requires additional development before real quantitative particle size distributions can be measured. 
APPENDIX B

TABULATION OF LEAK RATE EXPERIMENTS DATA 
TABLE B1. SUMMARY OF ROOM-TEMPERATURE PUO 2 LEAK RATE EXPERIMENTS USING A SIMULATED CRACK; (a) HELIUM FLOW RATE EQUAL TO $11.4 \mathrm{cc} / \mathrm{sec}$

\begin{tabular}{|c|c|c|c|c|c|c|c|c|}
\hline \multirow[b]{2}{*}{$\begin{array}{l}\text { Run } \\
\text { Number }\end{array}$} & \multirow[b]{2}{*}{$\begin{array}{l}\text { Tube } \\
\text { Rosition }\end{array}$} & \multirow{2}{*}{$\begin{array}{c}\text { HeIfum } \\
\text { Pressure, } \\
\text { paI }\end{array}$} & \multirow[b]{2}{*}{ Vibration } & \multirow{2}{*}{$\begin{array}{l}\text { Hellum } \\
\text { Leak } \\
\text { Rate(c) } \\
\text { scc/sec }\end{array}$} & \multirow{2}{*}{$\begin{array}{l}\text { Total } \\
\text { Hellum } \\
\text { Flow } \\
\text { ece (e) }\end{array}$} & \multicolumn{2}{|c|}{$\begin{array}{l}\text { Quantity of } \mathrm{PuO}_{2} \\
\text { Detected, } \mu \mathrm{g}(\mathrm{b})\end{array}$} & \multirow{2}{*}{$\begin{array}{c}\text { Nass Flow } \\
\text { Ratio } \\
\mu g / c c\end{array}$} \\
\hline & & & & & & Total & $\operatorname{Not}_{\text {Total }}^{(d)}$ & \\
\hline $\begin{array}{l}\mathrm{Pu} 4 \dot{6 b} \\
\mathrm{Pu} 46 \mathrm{c}\end{array}$ & $\begin{array}{l}\text { Up } \\
\text { Up }\end{array}$ & $\begin{array}{l}1000 \\
1000\end{array}$ & $\begin{array}{l}\text { No } \\
\text { No }\end{array}$ & $\begin{array}{l}7.8 \\
7.4\end{array}$ & $\begin{array}{l}4680 \\
4440\end{array}$ & $\begin{array}{l}0.0023 \\
0.0011\end{array}$ & $\begin{array}{l}-0.0002 \\
-0.0014\end{array}$ & $\begin{array}{l}-4.3 E-08 \\
-3.2 E-07\end{array}$ \\
\hline Pu $47 b$ & $U_{p}$ & 1000 & Yes & 7.8 & 4680 & 0.0013 & -0.0012 & $-2.6 \mathrm{E}-07$ \\
\hline Pu $47 c$ & Up & 1000 & Yes & 7.4 & 4440 & 0.0017 & -0.0008 & $-1.8 E-07$ \\
\hline Pu $48 d$ & Down & 1000 & No & 7.8 & 4680 & 0.0008 & -0.0017 & $-3.6 E-07$ \\
\hline Pu $48 \mathrm{e}$ & Down & 1000 & No & 7.4 & 4440 & 0.0015 & -0.0010 & $-2.3 E-07$ \\
\hline Pu 49d & $U_{p}$ & 500 & Yeg & 3.0 & 1800 & 0.0025 & 0.0000 & 0.0000 \\
\hline Pu 49 e & Up & 500 & Yes & 2.6 & 1560 & 0.0007 & -0.0007 & $-4.5 E-07$ \\
\hline $\mathrm{Pu} 49 \mathrm{~g}$ & $\mathrm{U}_{\mathrm{p}}$ & 500 & Yes & 2.6 & 1560 & 0.0019 & -0.0006 & $-3.8 E-07$ \\
\hline $\mathrm{Pu} 50 \mathrm{~b}$ & Down & 500 & Yes & 2.2 & 1320 & 0.0028 & 0.0003 & $2.3 E-07$ \\
\hline Pu 50 c & nown & 500 & Yes & 2.2 & 1320 & 0.0028 & 0.0003 & $2.3 E-07$ \\
\hline Pu 51d & $U_{p}$ & Ambient & Yes & - & - & 0.0035 & 0.0010 & \\
\hline Pu 51e & $U_{p}$ & Ambient & Yes & - & - & 0.0034 & 0.0009 & \\
\hline $\mathrm{Pu} 52 \mathrm{~b}$ & Down & Ambient & No & - & - & 0.0019 & -0.0006 & \\
\hline $\mathrm{Pu} 52 \mathrm{c}$ & Down & Ambient & No & - & - & 0.0010 & -0.0015 & \\
\hline
\end{tabular}

(a) The almulated crack has a 220-um finish perpendicular to the hellum flow. The hellum leak rate was initially $11.4 \mathrm{cc} / \mathrm{sec}$ at 920 paig. All runs were for $10 \mathrm{~min}$

(b) Based on a specific activity of $0.096 \mathrm{C1} / \mathrm{g}$ for the $\mathrm{PuO}$ powder.

(c) The leak rate was determined by the pressure decay method at the midpoint of the run.

(d) The net total 1o the amount above the average containment box background of $0.0025 \mathrm{\mu g}$.

(e) Best estimate of total flow in standard $\mathrm{CM}^{3}$ standard conditions: 1 atm $25^{\circ} \mathrm{C}$. 
TABLE B2. SUMMARY OF ROOM-TEMPERATURE PuO 2 LEAK RATE EXPERIMENTS USING A SIMULATED CRACK; (a) INITIAL HELIUM FLOW RATE EQUAL TO $9.8 \mathrm{cc} / \mathrm{sec}$ AT $1000 \mathrm{p}$ gi

\begin{tabular}{|c|c|c|c|c|c|c|c|c|}
\hline \multirow[b]{2}{*}{$\begin{array}{c}\text { Run } \\
\text { Number }\end{array}$} & \multirow[b]{2}{*}{$\begin{array}{l}\text { Tube } \\
\text { Position }\end{array}$} & \multirow{2}{*}{$\begin{array}{c}\text { Helium } \\
\text { Pressure, } \\
\text { psi }\end{array}$} & \multirow[b]{2}{*}{ Vibration } & \multirow{2}{*}{$\begin{array}{l}\text { Hel ium } \\
\text { I,eak }(c) \\
\text { Rate } \\
\text { scc/sec }\end{array}$} & \multirow{2}{*}{$\begin{array}{c}\text { Total } \\
\text { Helium } \\
\text { Flow }(e) \\
\end{array}$} & \multicolumn{2}{|c|}{$\begin{array}{l}\text { Quantity of } \mathrm{PuO}_{2} \\
\text { Detected, } \mathrm{\mu g}(\mathrm{h}) \\
\end{array}$} & \multirow[b]{2}{*}{$\begin{array}{c}\text { Nass Flow } \\
\text { Rat } 10 \\
\text { ug/ce }\end{array}$} \\
\hline & & & & & & Total & $\begin{array}{c}\text { Net } \\
\text { Total }\end{array}$ & \\
\hline $54 a$ & Up & 1000 & No & 8.7 & 5220 & 0.0054 & 0.0041 & $7.9 E-07$ \\
\hline $54 b$ & Up & 1000 & No & 8.7 & 5220 & 0.0056 & 0.0043 & $8.2 E-07$ \\
\hline 57 & Up & 1000 & Yes & 8.7 & 5220 & 0.0098 & 0.0085 & $1.6,06$ \\
\hline $57 a$ & Up & 1000 & Yes & 8.7 & 5220 & 0.0027 & 0.0014 & $2.7 \mathrm{E}-07$ \\
\hline 58 & Down & 1000 & No & 8.7 & 5220 & 0.0037 & 0.0024 & $4.6 E-07$ \\
\hline $58 a$ & Down & 1000 & No & 7.8 & 4680 & 0.0025 & 0.0012 & $2.6 E-07$ \\
\hline 59 & Up & 500 & Yes & 2.6 & 1560 & 0.0008 & -0.0005 & $-3.2 E-07$ \\
\hline $59 \mathbf{a}$ & Up & 500 & Yes & 2.6 & 1560 & 0.0041 & 0.0028 & $1.8 \mathrm{E}-06$ \\
\hline $59 \mathrm{~b}$ & Up & 500 & Yes & 2.6 & 1560 & 0.0003 & -0.0010 & $-6.4 E-07$ \\
\hline 53 & Down & 500 & Yes & 3.0 & 1800 & 0.0986 & 0.0973 & $5.4 \mathrm{E}-05$ \\
\hline $53 b$ & Down & 500 & Yes & 3.0 & 1800 & 0.0359 & 0.0346 & $1.9 E-05$ \\
\hline 55 & Up & Ambient & Yes & - & & 0.0062 & 0.0049 & \\
\hline $55 a$ & Up & Ambient & Yes & - & & 0.0019 & 0.0006 & \\
\hline 56 & Down & Ambient & Yes & - & & 0.0005 & -0.0008 & \\
\hline $56 a$ & Down & Ambient & Yes & - & & 0.0005 & -0.0008 & \\
\hline 60 & Down & Ambient & No & - & & 0.0014 & 0.0001 & \\
\hline $60 a$ & Down & Ambient & No & - & & 0.0005 & -0.0008 & \\
\hline
\end{tabular}

(a) The simulated crack has a 220-microlnch finish perpendicular to the helium flow. The helfum leak rate was initially $9.8 \mathrm{cc} / \mathrm{sec}$ at $1000 \mathrm{psi}$. $\Lambda 11$ runs were for $10 \mathrm{~min}$

(b) Based on a spectfic activity of $0.096 \mathrm{Ci} / \mathrm{g}$ for the $\mathrm{PuO}$ powder.

(c) The leak rate was determined by the pressure decay method at the midpoint of the run.

(d) The net total is the amount above the average containment box background of $0.00130 . \mu g$.

(e) Best estimate of total flow in standard $\mathrm{cm}^{3}$. Standard conditions: 1 atm, $25^{\circ} \mathrm{C}$. 
TABLE B3. SUMMARY OF ROOM-TEMPERATURE PUO 2 LEAK RATE EXPERIMENTS USING A SIMULATED CRACK; (a) INITIAL HELIUM FLOW RATE EQUAL TO $11.6 \mathrm{cc} / \mathrm{sec}$ AT $1000 \mathrm{psi}$

\begin{tabular}{|c|c|c|c|c|c|c|c|c|}
\hline \multirow[b]{2}{*}{$\begin{array}{l}\text { Run } \\
\text { Number }\end{array}$} & \multirow[b]{2}{*}{$\begin{array}{c}\text { Tube } \\
\text { Position }\end{array}$} & \multirow{2}{*}{$\begin{array}{c}\text { Helium } \\
\text { Presisiure, } \\
\text { pst }\end{array}$} & \multirow[b]{2}{*}{ Vibration } & \multirow{2}{*}{$\begin{array}{l}\text { Icllium } \\
\text { Leak } \\
\text { Rilte(c) } \\
\mathrm{scc} / \mathrm{sec}\end{array}$} & \multirow{2}{*}{$\begin{array}{l}\text { Total } \\
\text { Hel tum } \\
\text { Flow } \\
\text { scc (e) }\end{array}$} & \multicolumn{2}{|c|}{$\begin{array}{l}\text { Quantity of } \mathrm{PuO}_{3} \\
\text { Detected, } \mathrm{gg}(\mathrm{b}) \\
\end{array}$} & \multirow{2}{*}{$\begin{array}{c}\text { Mass Flow } \\
\text { Raltio } \\
\mu g / c c\end{array}$} \\
\hline & & & & & & Tota 1 & Notal $_{\text {Tet }}^{\text {(d) }}$ & \\
\hline Pu 61 & $u_{p}$ & 1000 & No & 9.3 & 5580 & 0.5685 & 0.5672 & $1.0 \mathrm{E}-04$ \\
\hline Pu 61a & Up & 1000 & No & 8.5 & 5100 & 0.1384 & 0.1371 & $2.7 \mathrm{E},-05$ \\
\hline Pu 61b & Up & 1000 & No & 6.5 & 3900 & 0.0136 & 0.0123 & 3. $2 \mathrm{E}-06$ \\
\hline Pu 61e & Up & 1000 & No & 6.9 & 4140 & 0.0043 & 0.0030 & $7.2 \mathrm{E}-07$ \\
\hline Pu 62 & Up & 1000 & Yes & 7.4 & 4440 & 1.750 & 1.749 & $3.9 E-04$ \\
\hline $\mathrm{Pu} \mathrm{62a}$ & Up & 1000 & Yee & 7.4 & 4440 & 0.0018 & 0.0005 & $1.1 \mathrm{E}-07$ \\
\hline $\mathrm{Pu}$ 62b & Up & 1000 & Yes & 6.9 & 4140 & 0.0131 & 0.0118 & $2.9 E-06$ \\
\hline $\mathrm{Pu} \mathrm{62c}$ & Up & 1000 & Yes & 6.9 & 4140 & 0.0028 & 0.0015 & $3.6 \mathrm{E}-07$ \\
\hline Pu 63 & Down & 1000 & No & 6.1 & 3660 & 0.2697 & 0.2684 & $7.3 E-05$ \\
\hline Pu 63a & Down & 1000 & No & 6.5 & 3900 & 0.4127 & 0.4114 & $1.1 \mathrm{E}-04$ \\
\hline $\mathrm{Pu}$ 63b & Down & 1000 & No & 6.9 & 4140 & 0.1445 & 0.1432 & $3.5 E-04$ \\
\hline $\mathrm{Pu} \mathrm{63c}$ & Down & 1000 & No & 6.9 & 4140 & 34.79 & 34.79 & 8. $4 E-03$ \\
\hline Pu 63d & Down & 1000 & No & 6.9 & 4140 & 0.0991 & 0.0978 & $2.4 \pi-05$ \\
\hline $\mathrm{Pu} 63 \mathrm{e}$ & Down & 1000 & No & 6.9 & 4140 & 0.0235 & 0.0222 & $5.4 \mathrm{E}-06$ \\
\hline Pu 64 & $U_{p}$ & 500 & Yes & 1.7 & 1020 & 0.0017 & 0.0004 & 3. $9 E-07$ \\
\hline Pu 64a & Up & 500 & Yes & 2.2 & 1320 & 0.0005 & -0.0008 & $-6.1 E-07$ \\
\hline Pu 65 & Down & 500 & Yes & 1.7 & 1020 & 1.742 & 1.741 & $1.7 \mathrm{E}-03$ \\
\hline Pu 65a & Down & 500 & Yes & 1.7 & 1020 & 0.1188 & 0.1175 & $1.2 \mathrm{E}-0 \mathrm{~S}$ \\
\hline $\mathrm{Pu} 65 \mathrm{~b}$ & Down & 500 & Yes & 2.2 & 1320 & 0.0030 & 0.0017 & 1. $3 E-06$ \\
\hline Pu 65c & Down & 500 & Yes & 2.2 & 1320 & 0.0109 & 0.0096 & 7. $3 E-06$ \\
\hline Pu 66 & $u_{p}$ & Ambient & Yes & - & & -0.0002 & -0.0015 & \\
\hline Pu 66a & $\mathrm{Up}_{\mathrm{p}}$ & Ambient & Yes & - & & 0.0004 & -0.0009 & \\
\hline Fu 67 & Down & Ambient & No & - & & 0.0022 & $0.000 y$ & . \\
\hline Eu 67a & Down & Ambient & No & - & & 0.0001 & -0.0012 & \\
\hline
\end{tabular}

(a) The simulated crack has a 220-microinch finish perpendicular to the hellum flow. The hellum leak rate was

$11.6 \mathrm{cc} / \mathrm{sec}$ at $1000 \mathrm{ps} 1$. A11 runs were for $10 \mathrm{~min}$

(b) Based on a specific activity of $0.096 \mathrm{Ci} / \mathrm{g}$ for the $\mathrm{PuO}_{2}$ powder.

(c) The leak rate was determined by the pressure decay method at the midpoint of the run. Standard conditions 1 atm, $25^{\circ} \mathrm{C}$

(d) The net total is the amount above the avergge containment box background of $0.0013 \mu 8$.

(e) Best estimate of total flow in standard $\mathrm{cm}^{3}$. Standard conditions: $1 \mathrm{~atm}, 25^{\circ} \mathrm{C}$. 
TABLE B4. SUMMARY OF ROOM-TEMPERATURE PuO 2 LEAR RATE EXPERIMENTS USING A SIMULATED CRACK; (a) INITIAL HELIUM FLOW RATE EQUAL TO $13.2 \mathrm{cc} / \mathrm{sec}$ AT $1000 \mathrm{psi}$

\begin{tabular}{|c|c|c|c|c|c|c|c|c|}
\hline \multirow[b]{2}{*}{$\begin{array}{l}\text { Run } \\
\text { Number }\end{array}$} & \multirow[b]{2}{*}{$\begin{array}{c}\text { Tube } \\
\text { Position }\end{array}$} & \multirow{2}{*}{$\begin{array}{c}\text { Helium } \\
\text { Pressure, } \\
\text { ps1 }\end{array}$} & \multirow[b]{2}{*}{ Vibration } & \multirow{2}{*}{$\begin{array}{l}\text { Hellum } \\
\text { Leak } \\
\text { Rate (c) } \\
\text { scc/sec }\end{array}$} & \multirow{2}{*}{$\begin{array}{l}\text { Total } \\
\text { Helium } \\
\text { Flow } \\
\text { scc (e) }\end{array}$} & \multicolumn{2}{|c|}{$\begin{array}{l}\text { Quantity of } \mathrm{PuO} \\
\text { Detected, } \mu \mathrm{g}(\mathrm{b})\end{array}$} & \multirow{2}{*}{$\begin{array}{c}\text { Mass Flow } \\
\text { Ratio } \\
\text { ng/cc }\end{array}$} \\
\hline & & & & & & Total & $\stackrel{\text { Net }}{\text { Total (d) }}$ & \\
\hline Pu 68 & Up & 1000 & No & 12.4 & 7440 & 0.1028 & 0.1015 & $1.4 E-05$ \\
\hline Pu $68 \mathrm{a}$ & Up & 1000 & No & 11.3 & 6780 & 0.0064 & 0.0051 & $7.5 E-07$ \\
\hline Pu 6BD & Up & 1000 & No & 9.6 & 5760 & 0.0038 & 0.0025 & 4. $3 E-07$ \\
\hline $\mathrm{Pu} 68 \mathrm{c}$ & Up & 1000 & No & 10.0 & 6000 & 0.0033 & 0.0020 & 3. $3 E-07$ \\
\hline Pu 69 & Up & 1000 & Yes & 11.1 & 6660 & 0.0083 & 0.0070 & $1.1 E-06$ \\
\hline $\mathrm{Pu} 69 \mathrm{a}$ & $U_{p}$ & 1000 & Yes & 9.3 & 5580 & 0.0050 & 0.0037 & $6.6 \mathrm{E}-07$ \\
\hline Pu 70 & Down & 1000 & No & 9.5 & 5700 & 0.0304 & 0.0291 & $5.1 E-06$ \\
\hline Pu $70 a$ & Down & 1000 & No & 9.7 & 5820 & 0.0546 & 0.0533 & $9.25-06$ \\
\hline $\mathrm{Pu} 71$ & Up & 500 & Yes & 3.0 & 1800 & 0.0048 & 0.0035 & $1.9 \mathrm{E}-06$ \\
\hline Pu $71 \mathrm{a}$ & Up & 500 & Yes & 2.6 & 1560 & 0.0006 & -0.0007 & $-4.5 E-07$ \\
\hline Pu 71b & Up & 500 & Yes & 2.6 & 1560 & 0.0017 & 0.0004 & 2.6E-07 \\
\hline Pu 71e & Up & 500 & Yes & 2.6 & 1560 & 0.0012 & -0.0001 & $-6.4 E-08$ \\
\hline $\mathrm{Pu} 72$ & Down & 500 & Yes & 2.6 & 1560 & 0.6522 & 0.6509 & $4.2 E-04$ \\
\hline $\mathrm{Pu} \mathrm{72a}$ & Down & 500 & Yes & 2.6 & 1560 & 0.4911 & 0.4898 & $3.1 E-04$ \\
\hline Pu 73 & Up & Amblent & Yes & - & & v.vuls & u. vuuv & \\
\hline Pu $73 a$ & Up & Ambient & Yes & - & & 0.0000 & -0.0013 & \\
\hline Pu 74 & Down & Amblent & No & - & & 0.1471 & 0.1458 & \\
\hline $\mathrm{Pu} 74 \mathrm{a}$ & Down & Ambient & No & - & & 0.0022 & 0.0009 & \\
\hline $\mathrm{Pu} 74 \mathrm{~b}$ & Down & Amblent & No & - & & 0.0019 & 0.0006 & \\
\hline $\mathrm{Pu} 74 \mathrm{c}$ & Down & Amb1ent & No & - & - & 0.0013 & 0.0000 & \\
\hline
\end{tabular}

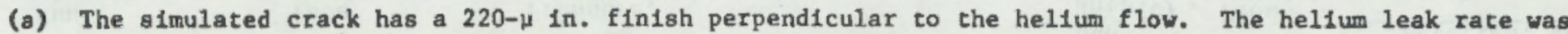
Initially $13.2 \mathrm{cc} / \mathrm{sec}$ at $1000 \mathrm{pol}$. All runs were for $10 \mathrm{~min}$.

(b) Based on a specific activity of $0.096 \mathrm{C} 1 / \mathrm{g}$ for the $\mathrm{PuO}$ powder.

(c) The leak rate was determined by the pressure decay method at the midpoint of the run. Standard conditions: 1 atm, $25^{\circ} \mathrm{C}$

(d) The net total 18 the amour.t above the average containment box background of $0.0013 \mu g$.

(e) Best estimate of total flow in stendard $\mathrm{cm}^{3}$. Standard conditions: 1 atm, $25^{\circ} \mathrm{C}$. 
TABLE B5. SUMMARY OF ROOM-TEMPERATURE PuO 2 LEAK RATE EXPERIMENTS USING A SIMULATED CRACK; (a) WITH AN INITIAL He FLOW RATE OF $17.3 \mathrm{cc} / \mathrm{sec}$ AT $1000 \mathrm{pgi}$

\begin{tabular}{|c|c|c|c|c|c|c|c|c|}
\hline \multirow[b]{2}{*}{$\begin{array}{l}\text { Ruo } \\
\text { kumber }\end{array}$} & \multirow[b]{2}{*}{$\begin{array}{l}\text { Tube } \\
\text { Poastion }\end{array}$} & \multirow{2}{*}{$\begin{array}{c}\text { Hellum } \\
\text { Pressure, } \\
\text { p81 }\end{array}$} & \multirow[b]{2}{*}{ Vibration } & \multirow{2}{*}{$\begin{array}{l}\text { Hellum } \\
\text { Leak } \\
\text { Rate(c), } \\
\text { ecc/sec }\end{array}$} & \multirow{2}{*}{$\begin{array}{l}\text { Total } \\
\text { Helium } \\
\text { Flow } \\
\text { Bcc (e) }\end{array}$} & \multicolumn{2}{|c|}{$\begin{array}{l}\text { Quantity of PuO } \\
\text { Detected, } \text { S }^{2} \mathrm{~b}^{2}\end{array}$} & \multirow[b]{2}{*}{$\begin{array}{c}\text { Mass Fiow } \\
\text { Raclo } \\
\mu g / c c \\
\end{array}$} \\
\hline & & & & & & Total & $\begin{array}{c}\text { Net } \\
\text { Total (d) }\end{array}$ & \\
\hline Pu 75 & Up & 1000 & No & 15.8 & 9480 & 0.0583 & 0.0570 & $6.0 \mathrm{E}-06$ \\
\hline Pu 75a & $U_{p}$ & 1000 & No & 16.3 & 9780 & 0.0287 & 0.0274 & $2.8 E-06$ \\
\hline Pu 76 & Up & 1000 & Yes & 15.8 & 9480 & 0.2029 & 0.2016 & $2.1 E-05$ \\
\hline Pu 76a & Up & 1000 & Yes & 16.3 & 9780 & 0.0160 & 0.0093 & $9.8 \mathrm{E}-07$ \\
\hline Pu 76b & $U_{p}$ & 1000 & Yeo & 8.25 & 4950 & 0.0137 & 0.0124 & $2.5 \mathrm{E}-06$ \\
\hline Pu $76 c$ & $U_{p}$ & 1000 & Yes & 8.25 & 4950 & 0.0047 & 0.0034 & $6.9 E-07$ \\
\hline ru 77 & Down & 1000 & No & 16.3 & 9780 & 0.0260 & 0.0247 & $2.5 E-06$ \\
\hline Pu $77 a$ & Down & 1000 & No & 16.3 & 9780 & 0.0237 & 0.0224 & 2. $3 E-06$ \\
\hline Pu 78 & Up & 500 & Yes & 2.6 & 1560 & 0.0058 & 0.0045 & $2.9 E-06$ \\
\hline Yu 78a & Up & 500 & Yes & 2.6 & 1560 & 0.0065 & 0.0052 & 3. $3 E-06$ \\
\hline Pu 79 & Down & 500 & Yes & 2.6 & 1560 & 0.4589 & 0.4576 & $2.9 E-04$ \\
\hline Pu $79 a$ & Down & 500 & Yes & 2.6 & 1560 & 0.6492 & 0.6479 & $4.2 E-04$ \\
\hline Pu 80 & Up & Ambient & Yes & - & & 0.0013 & 0.0000 & \\
\hline Pu 80a & Up & Amblent & Yes & - & & 0.0016 & 0.0003 & \\
\hline Pu 81 & Down & Amblent & No & - & & 0.0177 & 0.0164 & \\
\hline Pu 81a & Down & Amblent & No & - & & 0.0038 & 0.0025 & \\
\hline
\end{tabular}

(a) The simulated crack has a 220- $\mu$ in. finish perpendicular to the flow of the helium. The helium leak rate was Inttally $17.3 \mathrm{cc} / \mathrm{sec}$ at $1000 \mathrm{ps}$. A11 runs were for $10 \mathrm{~min}$.

(b) Based on a specific activity of $0.096 \mathrm{C1} / 8$ for the $\mathrm{PuO}$; powder.

(c) The leak tate was determined by the pressure decay method at the midpoint of the run. Standard cond1tipns: 1 atm, 25

(d) The net total is the amount above the avezage contalnment box background of $0.0013 \mathrm{wg}$.

(e) Best estimate of total flow in standard $\mathrm{cm}^{3}$. Standard conditions: 1 atm, $25^{\circ} \mathrm{C}$. 
TABLE B6. SUMMARY OF PuO 2 LEAK RATE EXPERIMENTS USING A 20- $\mu$ M ORIFICE(a)

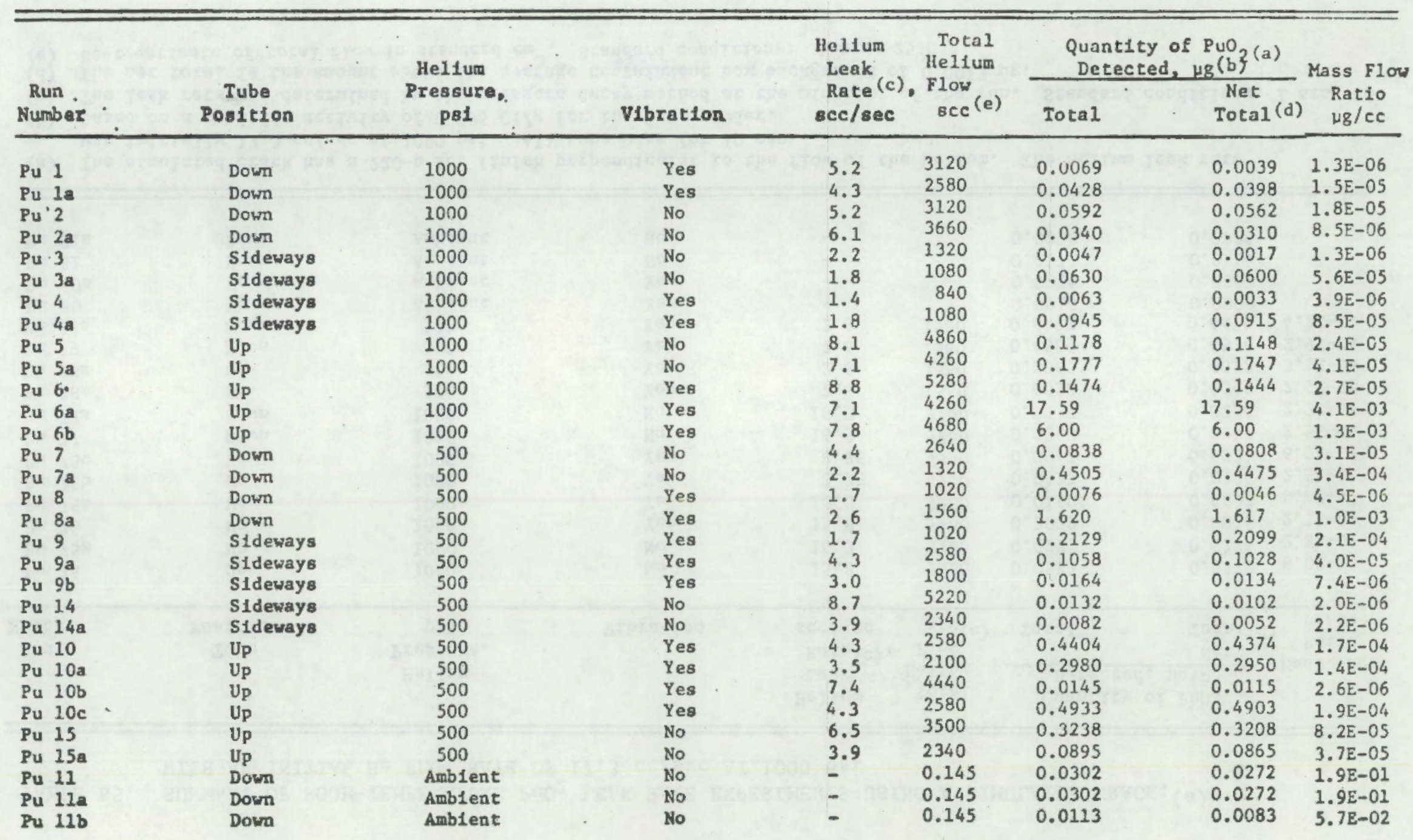


TABLE B6. SUMMARY OF PuO 2 LEAK RATE EXPERIMENTS USING A 20- $\mu$ m ORIFICE - Continued

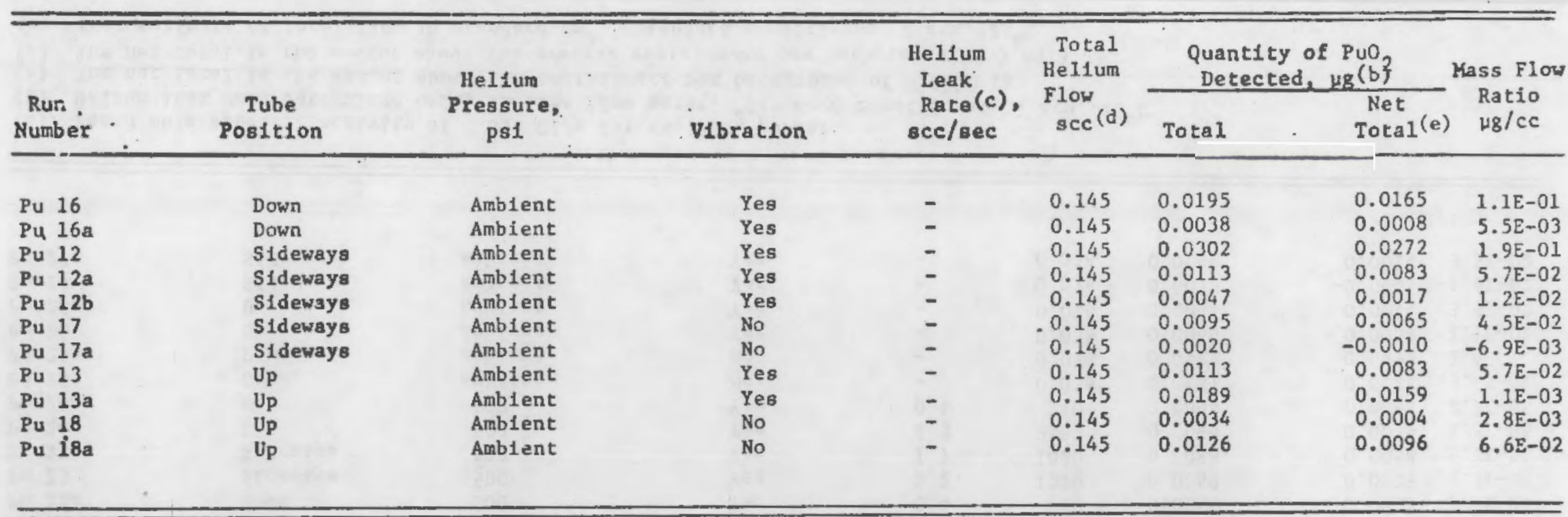

(a) Based on a specific activity of $0.096 \mathrm{CI} / 8$ for the $\mathrm{PuO}_{2}$ powder.

(b) Helium leak rate detemined using in-line flow meter. ${ }^{2}$ Standard conditions: 1 atm, $25^{\circ} \mathrm{C}$.

(c) The net total 18 the amount above the containment box background of $0.0030 \mu \mathrm{g}$.

(d) The net total is the amount above the averagge containment box background of $0.0013 \mu \mathrm{g}$.

(e) Best estimate of total flow in otandard $\mathrm{cm}$. Standard conditions: 1 atm, $25^{\circ} \mathrm{C}$. 
TABLE B7. SUMMARY OF PUO 2 LEAK RATE EXPERIMENTS USING A 10- $\mu \mathrm{m}$ ORIFICE(a)

\begin{tabular}{|c|c|c|c|c|c|c|c|c|}
\hline \multirow[b]{2}{*}{$\begin{array}{l}\text { Run } \\
\text { Number }\end{array}$} & \multirow[b]{2}{*}{$\begin{array}{l}\text { Tube } \\
\text { Position }\end{array}$} & \multirow{2}{*}{$\begin{array}{c}\text { Hel1um } \\
\text { Pressure } \\
\text { pa1 }\end{array}$} & \multirow[b]{2}{*}{ V1bration } & \multirow{2}{*}{$\begin{array}{l}\text { Helium } \\
\text { Leak } \\
\text { Rate (c), } \\
\text { scc/sec }\end{array}$} & \multirow{2}{*}{$\begin{array}{l}\text { Total } \\
\text { Hellum } \\
\text { Flow } \\
\text { sce (e) }\end{array}$} & \multicolumn{2}{|c|}{$\begin{array}{l}\text { Quantity of } \mathrm{PuO} \\
\text { Detected, } \mu \mathrm{g}^{(\mathrm{b})^{2}}\end{array}$} & \multirow{2}{*}{$\begin{array}{c}\text { Mass Flow } \\
\text { Rat1o } \\
\text { 1) } / \mathrm{cc}\end{array}$} \\
\hline & & & & & & Total & $\mathrm{Notal}_{\text {(d) }}^{\text {Net }}$ & \\
\hline $\mathrm{Pu} 19$ & Down & 1000 & No & 2.2 & 1320 & 0.2778 & 0.2748 & $2.1 E-04$ \\
\hline Pu 19a & Down & 1000 & No & 18.6 & 11160 & 0.0251 & 0.0221 & $2.0 E-06$ \\
\hline Pu' 20 & Sidewayo & 1000 & Yes & 1.3 & 780 & 0.0296 & 0.0266 & $3.4 E-05$ \\
\hline $\mathrm{Pu} 20 \mathrm{a}$ & S1deways & 1000 & Yes & 2.2 & $\begin{array}{r}1320 \\
11500\end{array}$ & 0.1341 & 0.1311 & $9.9 \mathrm{E}-05$ \\
\hline Pu 21 & Up & 1000 & Yes & 19.3 & 11580 & 0.4233 & 0.4203 & $3.6 \mathrm{E}-05$ \\
\hline Pu $21 a$ & Up & 1000 & Yes & 1.3 & 780 & 0.0088 & 0.0058 & $7.4 E-06$ \\
\hline $\mathrm{Pu} 21 \mathrm{~b}$ & $U_{p}$ & 1000 & Yes & 1.7 & 1020 & 0.0025 & $-0.0005=$ & $-4.9 E-07$ \\
\hline Pu 22 & Down & 500 & Yes & 0.9 & 540 & 0.0119 & 0.0089 & $1.6 E-05$ \\
\hline Pu 22a & Down & 500 & Yes & 0.4 & 240 & 0.0365 & 0.0335 & $1.4 \mathrm{E}-04$ \\
\hline Pu 23 & Sideways & 500 & Yes & 2.2 & 1320 & 0.0069 & 0.0039 & $1.1 \mathrm{E}-07$ \\
\hline Pu 23a & S1deways & 500 & Yes & 1.7 & 1020 & 0.0088 & 0.0058 & $5.7 \mathrm{E}-06$ \\
\hline Pu 24 & Up & 500 & Yes & 4.3 & 2580 & 0.0069 & 0.0039 & $1.5 \mathrm{E}-06$ \\
\hline $\mathrm{Pu} 24 \mathrm{a}$ & Up & 500 & Yes & 0.4 & 240 & 0.0094 & 0.0064 & 2. $7 \varepsilon-05$ \\
\hline Pu 25 & Down & Ambtent & No & - & 0.036 & 0.0151 & 0.0121 & $3.4 \mathrm{E}-01$ \\
\hline Pu 25a & Down & Ambient & No & - & 0.036 & 0.0522 & 0.0492 & 1.4 \\
\hline Pu 26 & $U_{p}$ & Ambient & Yes & - & 0.036 & 0.0069 & 0.0039 & $1.1 \mathrm{E}-01$ \\
\hline Pu $26 a$ & Up & Ambient & Yes & - & 0.036 & 0.0044 & 0.0014 & $3.3 E-02$ \\
\hline Pu 27 & Sideways & Ambient & Yes & - & 0.036 & 0.0025 & $-0.0005-$ & $-1.4 E-02$ \\
\hline Pu 27a & S1deways & Ambient & Yes & - & 0.036 & 0.0044 & 0.0014 & $3.9 E-02$ \\
\hline
\end{tabular}

(a) Based on a specific activity of $0.096 \mathrm{C} 1 / 8$ for the $\mathrm{PuO}_{2}$ pouder.

(c) The net total io the amount above the containment box background of $0.0030 \mu 8$.

(d) The net total is the amount above the averagge contafnment box background of $0.0013 \mathrm{\mu g}$.

(e) Best estimate of total flow in standard $\mathrm{cm}^{3}$. Standard conditions: 1 atm, $25^{\circ} \mathrm{C}$. 
TABLE B8. SUMMARY OF PuO 2 LEAK RATE EXPERIMENTS USING A 5- $\mu \mathrm{m}$ ORIFICE (a)

\begin{tabular}{|c|c|c|c|c|c|c|c|c|}
\hline \multirow[b]{2}{*}{$\begin{array}{l}\text { Run } \\
\text { Number }\end{array}$} & \multirow[b]{2}{*}{$\begin{array}{c}\text { Tube } \\
\text { Position }\end{array}$} & \multirow{2}{*}{$\begin{array}{c}\text { Hel1um } \\
\text { Pressure, } \\
\text { psi }\end{array}$} & \multirow[b]{2}{*}{ Vibration } & \multirow{2}{*}{$\begin{array}{l}\text { Hellum } \\
\text { Leak } \\
\text { Rate }{ }^{(c),} \\
\text { scc/sec }\end{array}$} & \multirow{2}{*}{$\begin{array}{l}\text { Total } \\
\text { Hellum } \\
\text { Flow } \\
\text { sce }(e)\end{array}$} & \multicolumn{2}{|c|}{$\begin{array}{l}\text { Quantity of } \mathrm{PuO}_{2} \\
\text { Detected, } \mathrm{ug}^{(\mathrm{a})^{2}}\end{array}$} & \multirow{2}{*}{$\begin{array}{c}\text { Mass Flow } \\
\text { natio } \\
\mu g / c c\end{array}$} \\
\hline & & & & & & Total & $\operatorname{Total}_{\text {Net }}$ & \\
\hline $\mathrm{Pu} 2 \dot{8}$ & Down & 1000 & No & $\cdot 7.4$ & 4440 & 0.0113 & 0.0083 & $1.9 E-06$ \\
\hline Pu $28 a$ & Down & 1000 & No & 2.6 & 1560 & 0.0063 & 0.0033 & 2.1E-06 \\
\hline $\mathrm{Pu} \quad 37 \star$ & Down & 1000 & No & 5.5 & 19800 & 0.0038 & -0.0113 & $-1.9 \varepsilon-07$ \\
\hline Pu 38* & Down & 1000 & No & 0.9 & 3240 & 0.0107 & -0.0043 & $-3.3 E-06$ \\
\hline Pu 29 & Sidewaya & 1000 & Yes & 1.3 & 780 & 0.0145 & 0.0115 & 1. $5 E-05$ \\
\hline Pu 29a & Sideways & 1000 & Yes & 14.9 & 8940 & 0.0038 & 0.0008 & $8.9 E-08$ \\
\hline Pu 30 & Up & 1000 & Yes & 3.5 & 2100 & 0.0050 & 0.0020 & $9.5 E-07$ \\
\hline $\mathrm{Pu} 30 \mathrm{a}$ & Up & 1000 & Yes & 6.1 & 3660 & 0.0009 & -0.0021 & $-5.7 E-05$ \\
\hline Pu 31 & Down & 500 & Yes & 0.4 & 240 & 0.0069 & 0.0039 & 1. $6 \mathrm{E}-05$ \\
\hline Pu $31 \mathrm{a}$ & Down & 500 & Yes & 1.3 & 780 & 0.0101 & 0.0071 & $9.1 \mathrm{E}-06$ \\
\hline Pu 32 & SIdeways & 500 & Yes & 1.3 & 780 & 0.0050 & 0.0020 & $2.6 E-06$ \\
\hline Pu 32a & SIdeways & 500 & Yes & 0.4 & 240 & 0.0044 & 0.0014 & $5.8 \mathrm{E}-06$ \\
\hline Pu 33 & $\mathrm{Up}$ & 500 & Yes & 1.3 & 780 & 0.0044 & 0.0014 & $1.8 \mathrm{E}-06$ \\
\hline $\mathrm{Pu} 33 \mathrm{a}$ & Up & 500 & Yes & 4.8 & 2880 & 0.0082 & 0.0052 & $1.8 \mathrm{E}-06$ \\
\hline Pu 34 & Down & Ambient & No & - & 0.009 & 0.0044 & 0.0014 & 1. $6 E-01$ \\
\hline Pu 34a & Down & Ambient & No & - & 0.009 & 0.0082 & 0.0052 & $5.8 E-01$ \\
\hline $\mathrm{Pu} 35$ & Up & Amblent & Yes & - & 0.009 & 0.0017 & -0.0013 & $-1.4 E-01$ \\
\hline Pu 35a & Up & Amblent & Yes & - & 0.009 & 0.0018 & -0.0012 & $\begin{array}{l}-1.4 E-01 \\
-1.3 E-01\end{array}$ \\
\hline Pu 36 & Sideways & Abmitent & Yes & - & 0.009 & 0.0164 & 0.0134 & 1.5 \\
\hline Pu 36a & S1deways & Amblent & Yes & - & 0.009 & 0.0113 & 0.0083 & $9.2 \mathrm{E}-01$ \\
\hline
\end{tabular}

(a) Based on a specific activity of $0.096 \mathrm{Cl} / 8$ for the PuO ${ }_{2}$ powder.

(b) Helium leak rate determined using 1n-1lne flow meter. ${ }^{2}$ Standard conditions: 1 atm, $25^{\circ} \mathrm{C}$.

(c) The net total is the amount above the containment box background of $0.0030 \mathrm{ug}$.

(d) The net total is the amount above the averzge containment box hackground of $0.0013 \mathrm{lg}$.

(e) Best estimate of total flow in standard $\mathrm{cm}^{3}$. Standard conditions: 1 atm, $25^{\circ} \mathrm{C}$. 
TABLE B9. SUMMARY OF PUO 2 LEAK RATE EXPERIMENTS USING A 5- $\mu$ m ORIFICE(a)

\begin{tabular}{|c|c|c|c|c|c|c|c|c|c|}
\hline \multirow[b]{2}{*}{$\begin{array}{l}\text { Run } \\
\text { Number }\end{array}$} & \multirow[b]{2}{*}{$\begin{array}{l}\text { Tube } \\
\text { Position }\end{array}$} & \multirow{2}{*}{$\begin{array}{c}\text { Hel1um } \\
\text { Pressure, } \\
\text { ps1 }\end{array}$} & & \multirow[b]{2}{*}{ Vibration } & \multirow{2}{*}{$\begin{array}{l}\text { Hellum } \\
\text { Leak } \\
\text { Rate(b) } \\
\text { scc/sec }\end{array}$} & \multirow{2}{*}{$\begin{array}{l}\text { Total } \\
\text { Helium } \\
\text { Flow } \\
\text { scc (e) }\end{array}$} & \multicolumn{2}{|c|}{$\begin{array}{l}\text { Quantity of } \mathrm{PuO}_{2} \\
\text { Detected, } \mu \mathrm{g}(\mathrm{a})^{2}\end{array}$} & \multirow{2}{*}{$\begin{array}{c}\text { Mass Flow } \\
\text { Rat } 10 \\
\mathrm{Hg} / \mathrm{cc}\end{array}$} \\
\hline & & & & & & & Total & $\begin{array}{c}\text { Net } \\
\text { Total (d) }\end{array}$ & \\
\hline & & & & . & . & & & . & \\
\hline Pu 82 & Up & Amblent & - & No & - & 0.009 & 0.0042 & 0.0029 & 0.32 \\
\hline $\mathrm{Pu} 82 \mathrm{a}$ & Up & Ambient & & No & - & 0.009 & 0.0059 & 0.0046 & 0.51 \\
\hline Pu 83 & Down & Ambient & & Yes & - & 0.009 & 0.0095 & 0.0082 & 0.91 \\
\hline Pu $83 a$ & Down & Ambient & & Yes & - & 0.009 & 0.0168 & 0.0155 & 1.72 \\
\hline Pu $83 b$ & Down & Amblent & & Yes & - & 0.009 & 0.1000 & 0.0987 & 10.97 \\
\hline $\begin{array}{l}\text { Pu } 83 c \\
\text { Pu } 84\end{array}$ & Down & Ambient & & Yes & - & 0.009 & 0.0064 & 0.0051 & 0.57 \\
\hline $\begin{array}{l}\text { Pu } 84 a \\
\text { Pu }\end{array}$ & Up & 1000 & & No & 0.4 & 240 & 0.0009 & -0.0004 & $-1.7 E-06$ \\
\hline Pu 85 & $\begin{array}{l}\text { Up } \\
\text { Down }\end{array}$ & 1000 & & No & 0.4 & 240 & 0.0008 & -0.0005 & $-2.1 E-06$ \\
\hline $\mathrm{Pu} 35 \mathrm{a}$ & $\begin{array}{l}\text { Down } \\
\text { Down }\end{array}$ & 1000 & & Yes & 0.4 & 240 & 0.0028 & 0.0015 & $6.3 E-06$ \\
\hline Pu. 86 & $\begin{array}{l}\text { Down } \\
\text { Down }\end{array}$ & 1000 & & Yes & 1.7 & 1020 & 0.0042 & 0.0029 & $2.8 \mathrm{E}-06$ \\
\hline Pu $86 a$ & $\begin{array}{l}\text { Down } \\
\text { Down }\end{array}$ & 1000 & & No & 3.9 & 2340 & 0.0134 & 0.0121 & 5. $2 \mathrm{E}-06$ \\
\hline Pu $86 b$ & $\begin{array}{l}\text { Down } \\
\text { Down }\end{array}$ & 1000 & & No & 0.9 & 540 & 0.0020 & 0.0007 & 1. $3 E-06$ \\
\hline Pu 86c & $\begin{array}{l}\text { Down } \\
\text { Down }\end{array}$ & 1000 & & No & 0.4 & 240 & 0.0024 & 0.0011 & 4. $6 \mathrm{E}-06$ \\
\hline & Down & 1000 & . & No & 0.4 & 240 & 0.0005 & -0.0008 & $-3.3 E-06$ \\
\hline
\end{tabular}

(a) Based on a spectflc activity of $0.096 \mathrm{Cl} / \mathrm{g}$ for the $\mathrm{PuO}_{2}$ powder.

(b) Helium leak rate determined using in-1ine fiow meter. 2 standard conditions: 1 atm, $25^{\circ} \mathrm{c}$.

(c) The net total is the amount above the contalnment box background of $0.0030 \mu \mathrm{g}$.

(e) Best estimate of total flow in standard $\mathrm{cm}^{3}$. Standard conditions: $1 \mathrm{~atm}, 25^{\circ} \mathrm{C}$. 
TABLE B10. SUMMARY OF PuO 2 LEAK RATE EXPERIMENTS USING A 10- $\mu \mathrm{m}$ ORIFICE(a)

\begin{tabular}{|c|c|c|c|c|c|c|c|c|}
\hline \multirow[b]{2}{*}{$\begin{array}{l}\text { Run } \\
\text { Number }\end{array}$} & \multirow[b]{2}{*}{$\begin{array}{l}\text { Tube } \\
\text { Poṣition }\end{array}$} & \multirow{2}{*}{$\begin{array}{c}\text { Hellum } \\
\text { Pressure, } \\
\text { pol }\end{array}$} & \multirow[b]{2}{*}{ Vibration } & \multirow{2}{*}{$\begin{array}{l}\text { Hellum } \\
\text { Leak } \\
\text { Rate (c), } \\
\text { scc/sec }\end{array}$} & \multirow{2}{*}{$\begin{array}{l}\text { Total } \\
\text { Hellum } \\
\text { Flow } \\
\text { scc }(e)\end{array}$} & \multicolumn{2}{|c|}{$\begin{array}{l}\text { Quantity of } \mathrm{PuQ} \\
\text { Detected, } \mu_{2}(\mathrm{~b})^{2}\end{array}$} & \multirow{2}{*}{$\begin{array}{c}\text { Mass Flow } \\
\text { Ratio } \\
\text { Hg/cc }\end{array}$} \\
\hline & & & & & & Total & $\operatorname{Not}_{\text {Total }}^{(d)}$ & \\
\hline Pu 87 & Down & 500 & Yes & 0.4 & 240 & 0.0071 & 0.0058 & $2.4 E-05$ \\
\hline Ṕu 87a & Down & 500 & Yes & 0.4 & 240 & 0.0019 & 0.0006 & 2. $5 E-06$ \\
\hline $\mathrm{Pu} 87 \mathrm{~b}$ & Down & 500 & Yes & 0.4 & 240 & 0.0019 & 0.0006 & 2. $5 E-06$ \\
\hline Pu 87c & Down & 500 & Yes & 0.4 & 240 & 0.0066 & 0.0053 & 2. $2 E-05$ \\
\hline $\mathrm{Pu} 88$ & Down & 500 & No & 0.4 & 240 & 0.0066 & 0.0053 & 2. $2 \mathrm{E}-05$ \\
\hline $\mathrm{Pu} 88 \mathrm{a}$ & Down & 500 & No & 0.4 & 240 & 0.0052 & 0.0039 & 1. SE-05 \\
\hline $\mathrm{Pu} 88 \mathrm{~b}$ & Down & 500 & No & 0.4 & 240 & 0.0003 & -0.0010 & $-4.2 E-06$ \\
\hline Pu $88 c$ & Up & 500 & No & 0.4 & 240 & 0.0012 & -0.0001 & $-4.2 E-07$ \\
\hline Pu 89 & Up & 500 & No & 0.8 & 480 & 0.0117 & 0.0104 & 2. $5 E-05$ \\
\hline Pu $89 a$ & Up & 500 & No & 0.8 & 480 & 0.0024 & 0.0011 & 2. $3 E-06$ \\
\hline Pu $89 b$ & Up & 500 & No & 0.8 & 480 & 0.0026 & 0.0013 & 2.7E-06 \\
\hline Pu 89c & Up & 500 & No & 0.8 & 480 & 0.0009 & -0.0004 & $-8.3 E-07$ \\
\hline
\end{tabular}

(a) Based on a specific activity of $0.096 \mathrm{Cl} / \mathrm{g}$ for the $\mathrm{PuO}_{2}$ powder.

(b) Helium leak rate determined using in-line flow meter. ${ }^{2}$ Standard conditions: 1 atm, $25^{\circ} \mathrm{C}$.

(c) The net total is the amount above the containment box background of $0.0030 \mu \mathrm{g}$.

(d) The net total is the amount above the average containment box background of $0.0013 \mu \mathrm{g}$.

(e) Best estimate of total flow in otandard $\mathrm{cm}^{3}$. Standard conditions: 1. atm, $25^{\circ} \mathrm{c}$. 
TABLE B11. SUMMARY OF PuO 2 LEAK RATE EXPERIMENTS USING A 8- $\mu$ m ORIFICE(a)

\begin{tabular}{|c|c|c|c|c|c|c|c|c|}
\hline \multirow[b]{2}{*}{$\begin{array}{l}\text { Run } \\
\text { Kumber }\end{array}$} & \multirow[b]{2}{*}{$\begin{array}{l}\text { Tube } \\
\text { Rosttion }\end{array}$} & \multirow{2}{*}{$\begin{array}{c}\text { Helfum } \\
\text { Pressure, } \\
\text { pal }\end{array}$} & \multirow[b]{2}{*}{ Vibration } & \multirow{2}{*}{$\begin{array}{l}\text { Helium } \\
\text { Leak } \\
\text { Rate(c) } \\
\text { scc/sec }\end{array}$} & \multirow{2}{*}{$\begin{array}{l}\text { Total } \\
\text { Helium } \\
\text { Flow } \\
\text { Bcc (e) }\end{array}$} & \multicolumn{2}{|c|}{$\begin{array}{l}\text { Quantity of PuO, } \\
\text { Detected, } \mathrm{ug}^{(\mathrm{b})}\end{array}$} & \multirow[b]{2}{*}{$\begin{array}{c}\text { Mass Flow } \\
\text { Ratio } \\
\mu g / c c \\
\end{array}$} \\
\hline & & & & & & Total. & $\begin{array}{c}\text { Net } \\
\text { Total'(d) }\end{array}$ & \\
\hline $\mathrm{Pu} 98$ & Up & 1000 & Yes & 5.2 & 3120 & 0.1113 & 0.1100 & $3.5 E-05$ \\
\hline $\mathrm{Pu} \cdot 98 \mathrm{a}$ & $U_{p}$ & 1000 & Yes & 3.0 & 1800 & 0.0082 & 0.0069 & $3.8 E-06$ \\
\hline Pu 98b & Up & 1000 & Yes & 23.7 & 14220 & 0.0020 & 0.0007 & $4.9 E-08$ \\
\hline Pu $98 c$ & Up & 1000 & Yes & 1.1 & 660 & 0.0019 & 0.0006 & $9.1 \mathrm{E}-07$ \\
\hline Pu 99 & Down & 1000 & Yes & 0.87 & 520 & 0.0684 & 0.0671 & 1. $3 E-04$ \\
\hline Pu 99a & Down & 1000 & Yes & 1.1 & 660 & 0.0014 & 0.0001 & $1.5 \mathrm{E}-07$ \\
\hline Pu 99b & Down & 1000 & Yes & 1.1 & 660 & 0.0012 & -0.0001 & $-1.5 \mathrm{E}-07$ \\
\hline Pu 99c & Down & 1000 & Yes & 2.2 & 1320 & 0.0057 & 0.0044 & 3. $3 E-06$ \\
\hline Pu 100 & Sideways & 1000 & Yes & $\cdot 1.1$ & 660 & 0.0016 & 0.0003 & $4.5 E-07$ \\
\hline Pu $100 a$ & Sideways & 1000 & Yeg & 1.1 & 660 & 0.0021 & 0.0008 & 1. $2 E-06$ \\
\hline Pu $100 \mathrm{~b}$. & S1deways & 1000 & Yes & 2.2 & 1320 & 0.0024 & 0.0011 & 8. $3 E-07$ \\
\hline Pu $100 c^{\circ}$ & Sideway & 1000 & Yes & 2.2 & 1320 & 0.0017 & 0.0004 & 3.0E -07 \\
\hline$\star$ Pu 101 & Down & 1000 & No & 1.3 & 4680 & 0.0654 & 0.0641 & $1.4 E-05$ \\
\hline$\star P u \quad 101 a$ & Down & 1000 & No & 4.8 & 17280 & 0.0082 & 0.0069 & $4.0 E-07$ \\
\hline$+\mathrm{Pu} 102$ & Down & 1000 & No & 1.1 & 7920 & 0.0024 & 0.0011 & $1.4 E-07$ \\
\hline$+P u \quad 102 a$ & Down & 1000 & No & 7.8 & 56160 & 0.0030 & -0.0017 & $-3.0 E-08$ \\
\hline Pu 103 & $\mathrm{Up}_{\mathrm{p}}$ & 1250 & Yes & 24.8 & 14880 & 0.0033 & 0.0020 & 1. $3 E-07$ \\
\hline Pu $103 a$ & vp & 1250 & Yes & 1.3 & 780 & 0.0009 & -0.0004 & $-5.1 E-07$ \\
\hline Pu 104 & Down & 1250 & Yes & 2.2 & 1320 & 0.0008 & -0.0005 & $-3.8 E-07$ \\
\hline Pu $104 a$ & Down & 1250 & Yes & 6.9 & 24840 & 0.0018 & 0.0005 & $2.0 E-08$ \\
\hline APu 105 & Down & 1250 & No & 1.3 & 4680 & 0.0008 & -0.0005 & $-1.1 \varepsilon-07$ \\
\hline •Pu 105a & Down & 1250 & No & 1.7 & 12240 & 0.0035 & 0.0022 & $1.8 \mathrm{E}-07$ \\
\hline +Pu 106 & Down & 1250 & No & $2: 2$ & 15840 & 0.0017 & 0.0004 & $2.5 E-08$ \\
\hline +Pu $106 a$ & Down & 1250 & No. & 10.9 & 78480 & 0.0013 & 0.0000 & 0.0000 \\
\hline
\end{tabular}

(a) Based on a specific activity of $0.096 \mathrm{Cl} / 8$ for the $\mathrm{PuO}_{2}$ powder.

(b) Helium leak rate determined using 1n-1ine flow meter. ${ }^{2}$ standard conditions: 1 atm, $25^{\circ} \mathrm{C}$.

(c) The net total 18 the amount above the containment box background of $0.0030 \mathrm{\mu g}$.

(d) The net total is the amount above the average containment box background of $0.0013 \mu g$.

(e) Best estimate of total flow in standard $\mathrm{cm}$. Standard conditions: 1 atm, $25^{\circ} \mathrm{C}$. 
TABLE B12. SUMMARY OF PUO 2 LEAK RATE EXPERIMENTS USING A 20- $\mu$ m ORIFICE(a)

\begin{tabular}{|c|c|c|c|c|c|c|c|c|}
\hline \multirow{2}{*}{$\begin{array}{l}\text { Run } \\
\text { Number }\end{array}$} & \multirow{2}{*}{$\begin{array}{c}\text { Tube } \\
\text { Position }\end{array}$} & \multirow{2}{*}{$\begin{array}{c}\text { Helium } \\
\text { Presoure, } \\
\text { ps1 }\end{array}$} & \multirow[b]{2}{*}{ Vibration } & \multirow{2}{*}{$\begin{array}{l}\text { Helium } \\
\text { Leak (c) } \\
\text { Rate } \\
\text { scc/sec }\end{array}$} & \multirow{2}{*}{$\begin{array}{l}\text { Total } \\
\text { Hellum } \\
\text {, Flow } \\
\sec ^{(e)}\end{array}$} & \multicolumn{2}{|c|}{$\begin{array}{l}\text { Quantity of PuO }{ }^{2} \\
\text { Detected, } \mathrm{Hg}_{\mathrm{g}}(\mathrm{b})^{2}\end{array}$} & \multirow{2}{*}{$\begin{array}{l}\text { Mass Flo } \\
\text { Ratio } \\
\mu g / c c \\
\end{array}$} \\
\hline & & & & & & Tota1 & $\begin{array}{c}\text { Net } \\
\text { Total }\end{array}$ & \\
\hline Pu90 & Up & Ambient & Yes & - & 0.0071 & 0.0058 & 4. $0 \mathrm{E}-02$ & 0.145 \\
\hline PPu90a & Up & Ambient & Yes & - & 0.0022 & 0.0009 & $6.2 \mathrm{E}-03$ & 0.145 \\
\hline Pu91 & Down & Ambient & Yes & - & 0.0025 & 0.0012 & 8. $3 E-03$ & 0.145 \\
\hline Pugla & Down & Ambient & Yes & - & 0.0016 & 0.0003 & $2.1 E-03$ & 0.145 \\
\hline Pu92 & Up & Amblent & No & - & 0.0029 & 0.0016 & 1.1E-02 & 0.145 \\
\hline Pu92a & Up & Amblent & No & - & 0.0007 & -0.0006 & $-4.1 E-03$ & 0.145 \\
\hline Pu93 & Down & Ambient & No & - & 0.0044 & 0.0031 & $2.1 \varepsilon-03$ & 0.145 \\
\hline Pu93a & Down & Ambient & No & - & 0.0014 & 0.0001 & $6.9 E-04$ & 0.145 \\
\hline P494 & Up & 1000 & Yes & 6.5 & 0.0190 & 0.0177 & 4. $5 \mathrm{E}-06$ & 3900 \\
\hline Pu94a & Up & 1000 & Yes & 3.9 & 0.0006 & -0.0007 & $-3.0 E-07$ & 2340 \\
\hline Pu94b & Up & 1000 & Yes & 3.9 & 0.0014 & 0.0001 & 4. $3 \mathrm{E}-08$ & 2340 \\
\hline Pu94c & Up & 1000 & Yes & 3.9 & 0.0020 & 0.0007 & 3. $0 \mathrm{E}-07$ & 2340 \\
\hline Pu95 & Down & 1000 & Yes & 3.9 & 0.0014 & 0.0001 & 4. $3 E-08$ & 2340 \\
\hline Pu95a & Down & 1000 & Yes & 3.9 & 0.0238 & 0.0225 & $9.6 E-06$ & 2340 \\
\hline Pu95b & Down & 1000 & Yes & 3.9 & 0.0138 & 0.0125 & $5.3 E-06$ & 2340 \\
\hline Pu9 5c & Down & 1000 & Yes & 3.9 & 0.0017 & 0.0004 & 1. $7 E-07$ & 2340 \\
\hline Pu96 & Up & 1000 & No & 9.5 & 0.0022 & 0.0009 & $3.8 E-07$ & 2340 \\
\hline Pu96a & Up & 1000 & No & 3.5 & 0.0006 & -0.0007 & $-3.0 \mathrm{E}-07$ & 2340 \\
\hline Pu97 & Down & 1000 & No & 14.1 & 0.0021 & 0.0008 & $3.4 E-07$ & 2340 \\
\hline Pu97a & Down & 1000 & No & 3.9 & 0.0037 & 0.0024 & $1.0 E-06$ & 2340 \\
\hline Pu107 & Up & 1000 & No & 6.1 & 0.0814 & 0.0801 & $2.2 E-05$ & 3660 \\
\hline Pu107a & $U_{p}$ & 1000 & No & 2.2 & 0.0173 & 0.0160 & $1.2 E-05$ & 1320 \\
\hline Pul07b & Up & 1000 & No & 2.4 & 0.0053 & 0.0040 & $2.8 E-06$ & 1440 \\
\hline Pul07c & Up & 1000 & No & 10.6 & 0.0020 & 0.0007 & $1.1 \mathrm{E}-07$ & 6360 \\
\hline Pul07d & Up & 1000 & No & 2.6 & 0.0013 & 0.0000 & 0.0000 & 1560 \\
\hline Pul07e & Up & 1000 & No & 3.0 & 0.0233 & 0.0220 & 1. $2 \mathrm{E}-05$ & 1800 \\
\hline Pul07f & Up & 1000 & No & 2.2 & 0.0051 & 0.0038 & $2.9 E-06$ & 1320 \\
\hline Pu107g & Up & 1000 & No & 13.4 & 0.0498 & 0.0485 & $6.0 E-06$ & 8040 \\
\hline Pul07h & lip & 1000 & No & 6.5 & 0.0015 & 0.0002 & $5.1 E-08$ & 3900 \\
\hline Pul071 & Up & 1000 & No & 2.6 & 0.0020 & 0.0007 & $4.5 E-07$ & 1560 \\
\hline
\end{tabular}


TABLE B12. SUMMARY OF PuO 2 LEAK RATE EXPERIMENTS USING A 20- $\mu$ m ORIFICE - Continued

\begin{tabular}{|c|c|c|c|c|c|c|c|c|}
\hline \multirow[b]{2}{*}{$\begin{array}{l}\text { Run } \\
\text { Number }\end{array}$} & \multirow[b]{2}{*}{$\begin{array}{l}\text { Tube } \\
\text { Position }\end{array}$} & \multirow{2}{*}{$\begin{array}{c}\text { Hellum } \\
\text { Pressure, } \\
\text { psi }\end{array}$} & \multirow[b]{2}{*}{ Vibration } & \multirow{2}{*}{$\begin{array}{l}\text { HeJium } \\
\text { Leak (c) } \\
\text { Rate } \\
\text { scc/sec }\end{array}$} & \multirow{2}{*}{$\begin{array}{l}\text { Total } \\
\text { Helium } \\
\text { Flow } \\
\operatorname{scc}(e)\end{array}$} & \multicolumn{2}{|c|}{$\begin{array}{l}\text { Quantity of PuO }{ }^{2} \\
\text { Detected, } \mu \mathrm{g}(\mathrm{b})^{2}\end{array}$} & \multirow{2}{*}{$\begin{array}{c}\text { Mass Flow } \\
\text { Ratio } \\
\mu g / c c\end{array}$} \\
\hline & & & & & & Total & Total $(d)$ & \\
\hline Pu107j & Up & 1000 & No & 8.3 & 4980 & 0.0012 & -0.0001 & $-2.0 E-08$ \\
\hline Pu107k & Up & 1000 & No & 6.5 & 3900 & 0.0014 & 0.0001 & $2.6 \mathrm{E}-08$ \\
\hline Pul071 & Up & 1000 & No & 16.8 & 10080 & 0.0004 & -0.0009 & $-8.9 E-08$ \\
\hline Pu108m & Up & 1000 & No & 5.2 & 3120 & 0.0016 & 0.0003 & $9.6 \mathrm{E}-0 \mathrm{~B}$ \\
\hline Pu107n & Up & 1000 & No & 2.2 & 1320 & 0.0019 & 0.0006 & $4.5 E-07$ \\
\hline${ }_{x}^{x}$ Pu108 & Up & 1000 & No & 1.7 & & 0.1028 & 0.0963 & $1.6 \varepsilon-05$ \\
\hline${ }^{x} P u 108 a$ & Up & 1000 & No & 16.8 & & 0.0068 & 0.0003 & $5.0 \mathrm{E}-06$ \\
\hline${ }^{x}$ Pul08b & Up & 1000 & No & 5.6 & & 0.0129 & 0.0064 & $3.2 E-07$ \\
\hline${ }_{x}^{x}$ Pu108c & Up & 1000 & No & 2.6 & & 0.0011 & -0.0054 & $-5.8 E-07$ \\
\hline${ }^{x}$ Pu108d & Up & 1000 & No & 3.9 & & 0.0039 & 0.0026 & $1.9 \mathrm{E}-07$ \\
\hline "Pul09. & Up & 1000 & No & - & & 0.0078 & 0.0074 & 2. $5 E-05$ \\
\hline ^Pu109a & $U_{p}$ & 1000 & No & - & & 0.0013 & 0.0009 & $3.0 E-06$ \\
\hline *Pul09b & $\mathrm{up}$ & 1000 & No & - & & 0.0031 & 0.0027 & $9.0 E-06$ \\
\hline *Pul09c & Up & 1000 & No & - & & 0.0011 & 0.0007 & $2.3 E-06$ \\
\hline ^Pu109d & Up & 1000 & No & $=$ & & 0.0028 & 0.0024 & $8.0 E-06$ \\
\hline +Pu110 & Up & 1000 & No & 1.3 & & 0.0455 & 0.0325 & 3. $5 \mathrm{E}-06$ \\
\hline +Pul10a & Up & 1000 & No & 24.3 & & 0.0076 & -0.0054 & $-3.1 \mathrm{E}-08$ \\
\hline tPul10b & $u_{p}$ & 1000 & No & 0.9 & & 0.0038 & -0.0092 & $-1.4 \mathrm{E}-06$ \\
\hline$+P u 110 c$ & $\mathrm{Up}_{\mathrm{p}}$ & 1000 & No & 1.7 & & 0.0370 & 0.0240 & 2. $0 \mathrm{E}-06$ \\
\hline +Pul10d & Up & 1000 & No & 1.7 & & 0.0083 & -0.0047 & $-3.8 \mathrm{E}-07$ \\
\hline
\end{tabular}

(a) Based on a specific activity of $0.096 \mathrm{Ci} / \mathrm{g}$ for the $\mathrm{PuO}_{2}$ powder.

(b) Heḷum leak rate determined using in-line flow meter. ${ }^{2}$ Standard conditions: 1 atm, $25^{\circ} \mathrm{c}$.

(c) The net total is the amount above the contalnment box background of $0.0030 \mathrm{~kg}$.

(d) The net total is the amount above the average containment box background of $0.0013 \mu$.

(e) Best estimate of total flow in standard $\mathrm{cm}$. Standard conditions: 1 atm, $25^{\circ} \mathrm{C}$.

* 1 hr. collection time

"zero-time" runs

$+2 \mathrm{hr}$. collection time 
TABLE B13. SUMMARY OF $\mathrm{PuO}_{2}$ LEAK RATE EXPERIMENTS USING A 50- $\mu \mathrm{m}$ ORIFICE(a)

\begin{tabular}{|c|c|c|c|c|c|c|c|c|}
\hline \multirow[b]{2}{*}{$\begin{array}{l}\text { Run } \\
\text { Number }\end{array}$} & \multirow[b]{2}{*}{$\begin{array}{l}\text { Tube } \\
\text { Position }\end{array}$} & \multirow{2}{*}{$\begin{array}{c}\text { Hellum } \\
\text { Pressure. } \\
\text { pol }\end{array}$} & \multirow[b]{2}{*}{ Vibration } & \multirow{2}{*}{$\begin{array}{l}\text { Hellum } \\
\text { Leak (c) } \\
\text { Rate } \\
\text { scc/sec }\end{array}$} & \multirow{2}{*}{$\begin{array}{l}\text { Total } \\
\text { Hellum } \\
\text { Flow } \\
\text { sce (e) }\end{array}$} & \multicolumn{2}{|c|}{$\begin{array}{l}\text { Quant1ty of Puo }{ }^{2} \\
\text { Detected, } \mu \mathrm{g}(\mathrm{b})^{2}\end{array}$} & \multirow{2}{*}{$\begin{array}{c}\text { Mass Flow } \\
\text { Ratio } \\
\mu g / c c\end{array}$} \\
\hline & & & & & & Total & $\operatorname{Net}_{\text {Total }}^{(d)}$ & \\
\hline Pu. i11 & $U_{p}$ & 1000 & Yes & 8.7 & 5220 & 0.0006 & -0.0007 & $-1.3 E-07$ \\
\hline Pu 11la & Up & 1000 & Yes & 37.2 & 22320 & 0.0007 & -0.0006 & $-2.7 \mathrm{E}-08$ \\
\hline Pu 1118 & Up & 1000 & Yes & 2.6 & 1560 & 0.0007 & -0.0009 & $-3.8 E-07$ \\
\hline Pu 111e & Up & 1000 & Yes & 3.5 & 2100 & 0.0022 & 0.0009 & $4.3 \mathrm{E}-07$ \\
\hline Pu 112 & Down & 1000 & Yes & 2.6 & 1560 & 0.0179 & 0.0166 & $1.1 \mathrm{E}-05$ \\
\hline Pu $112 a$ & Down & 1000 & Yes & 3.5 & 2100 & 0.0009 & -0.0004 & $-1.9 E-07$ \\
\hline Pu $112 b$ & Down & 1000 & Yes & 2.6 & 1560 & 0.0005 & -0.0008 & $-5.1 E-07$ \\
\hline Pu 113 & Up & 500 & Yes & 1.3 & 780 & 0.0014 & 0.0001 & 1. $3 E-07$ \\
\hline Pu $113 a$ & Up & 500 & Yes & 1.3 & 780 & -0.0002 & -0.0015 & $-1.9 E-06$ \\
\hline Pu 114 . & Down & 500 & Yes & 1.3 & 780 & 0.0293 & 0.0280 & $3.6 E-05$ \\
\hline Pu $114 a$. & Down & 500 & Y̌es & 1.3 & 780 & 0.0005 & -0.0008 & $-1.0 E-06$ \\
\hline $\mathrm{Pu} 114 \mathrm{~b}$ & Dowa & 500 & Yes & 1.3 & 780 & 0.0011 & 0.0002 & $-2.6 E-07$ \\
\hline $\mathrm{Pu} 11 \mathrm{~s}$ & Up & 1250 & Yes & 4.8 & 2880 & 0.0034 & 0.0021 & 7. $3 \mathrm{E}-07$ \\
\hline $\mathrm{Pu} 115 \mathrm{a}$ & Up & 1250 & Yes & 4.8 & 2880 & 0.0007 & -0.0006 & $-2.1 \varepsilon-07$ \\
\hline Pu 116 & Down & 1250 & Yes & 5.4 & 3240 & 0.0006 & -0.0007 & $-2.2 \mathrm{E}-07$ \\
\hline Pu $116 a$ & Down & 1250 & Yes & 5.2 & 3120 & 0.0035 & 0.0022 & $7.1 E-07$ \\
\hline Pu 117 & $U_{p}$ & Ambient & Yes & - & 0.90 & 0.0008 & -0.0005 & $-5.6 \Xi-04$ \\
\hline Pu $117 a$ & $U_{P}$ & Ambient & Yes & - & 0.90 & 0.0019 & 0.0006 & $6.7 \varepsilon-04$ \\
\hline Pu 118 & Down & Ambient & Yes & - & 0.90 & 0.0059 & 0.0046 & $5.1 E-03$ \\
\hline Pu 118a & Down & Ambient & Yes & - & 0.90 & 0.0022 & 0.0009 & $1.0 \mathrm{E}-03$ \\
\hline
\end{tabular}

\footnotetext{
(a) Based on a specific activity of $0.096 \mathrm{Cl} / \mathrm{s}$ for the $\mathrm{PuO}_{2}$ powder.

(b) Helium leak rate determined using in-line flow meter. ${ }^{2}$ Standard conditions: 1 atm, $25^{\circ} \mathrm{C}$.

(c) The net total is the amount above the containment box background of $0.0030 \mu g$.

(d) The net cotal is the amount above the averąqe containment box hackground of $0.0013 \mu \mathrm{g}$.

(e) Best estimate of total flow in standard $\mathrm{cm}^{3}$. Standard conditions: 1 atm, $25^{\circ} \mathrm{C}$.
} 
TABLE B14. SUMMARY OF PUO 2 LEAK RATE EXPERIMENTS USING A 50- $\mu$ m ORIFICE(a)

\begin{tabular}{|c|c|c|c|c|c|c|c|c|}
\hline \multirow[b]{2}{*}{$\begin{array}{l}\text { Run } \\
\text { Number }\end{array}$} & \multirow[b]{2}{*}{$\begin{array}{c}\text { Tube } \\
\text { Position }\end{array}$} & \multirow{2}{*}{$\begin{array}{c}\text { Hellum } \\
\text { Pressure, } \\
\text { ps1 }\end{array}$} & \multirow[b]{2}{*}{ V1bration } & \multirow{2}{*}{$\begin{array}{l}\text { Hellum } \\
\text { Leak (c) } \\
\text { Rate }^{\text {(c) }} \\
\text { scc/sec }\end{array}$} & \multirow{2}{*}{$\begin{array}{l}\text { Total } \\
\text { Hellum } \\
\text { Flow } \\
\operatorname{scc}(\text { e) }\end{array}$} & \multicolumn{2}{|c|}{$\begin{array}{c}\text { Quant1ty of Pu0.2 } \\
\text { Detecred, } \mu \mathrm{g}(\mathrm{b})\end{array}$} & \multirow{2}{*}{$\begin{array}{c}\text { Mass Flow } \\
\text { Ratio } \\
\text { } \mathrm{g} / \mathrm{cc}\end{array}$} \\
\hline & & & & & & Tota1 & $\begin{array}{c}\text { Net } \\
\text { Total }\end{array}$ & \\
\hline Pu. 19 & Up & 1000 & Yès & 49.6 & 29760 & 12.80 & 12.80 & $4.3 E-04$ \\
\hline $\mathrm{Pu} 119 \mathrm{a}$ & Up & 1000 & Yes & 50.0 & 30000 & 7.206 & 7.20 & 1. $9 E-03$ \\
\hline Pui 1198 & Up & 1000 & Yes & 50.0 & 30000 & 2.4934 & 2.492 & $8.3 E-05$ \\
\hline Pu $119 c$ & Up & 1000 & Yes & 50.4 & 30240 & 0.9838 & 0.9825 & 3. $2 E-05$ \\
\hline Pu 119d & $U_{p}$ & 1000 & Yes & 50.7 & 30420 & 1.424 & 1.423 & $4.7 \mathrm{E}-0.5$ \\
\hline Pu 119e & Up & 1000 & Yes & $\begin{array}{r}50.8 \\
14.8\end{array}$ & 30480 & $\begin{array}{l}0.3374 \\
0.0065\end{array}$ & $\begin{array}{l}0.3361 \\
0.0052\end{array}$ & $1.1 E-05$ \\
\hline $\mathrm{Pu} 130 \mathrm{a}$ & Down & $\begin{array}{r}1000 \\
500\end{array}$ & Yes & $\begin{array}{r}103.1 \\
47.8\end{array}$ & 61860 & 7.974 & 7.973 & 8. $3 E-07$ \\
\hline Pu 120 & Up & $\begin{array}{l}500 \\
500\end{array}$ & $\begin{array}{l}\text { Yes } \\
\text { Yes }\end{array}$ & $\begin{array}{l}47.8 \\
47.4\end{array}$ & 28680 & $\begin{array}{l}2.468 \\
2.468\end{array}$ & 2.467 & $2.8 \mathrm{E}-04$ \\
\hline Pu $120 a$ & Up & 500 & $\begin{array}{l}\text { Yes } \\
\text { Yes }\end{array}$ & $\begin{array}{r}47.4 \\
7.4\end{array}$ & 28440 & $\begin{array}{l}2.408 \\
3.427\end{array}$ & $\begin{array}{l}2.461 \\
3.426\end{array}$ & 8. $7 E-05$ \\
\hline $\mathrm{Pu} 121$ & Luwn & 500 & Yes & $\begin{array}{l}7.4 \\
7.4\end{array}$ & 4440 & 0.0078 & $\begin{array}{l}3.426 \\
0.0065\end{array}$ & $7.7 \mathrm{E}-04$ \\
\hline $\mathrm{Pu} 121 \mathrm{a}$ & Down & 500 & Yes & & 4440 & $\begin{array}{l}0.0078 \\
0.0019\end{array}$ & $\begin{array}{l}0.0065 \\
0.0006\end{array}$ & 1. $5 \mathrm{E}-06$ \\
\hline $\mathrm{Pu} 122$ & Up & 500 & $\begin{array}{l}\text { No } \\
\text { No }\end{array}$ & 7.4 & 4440 & $\begin{array}{l}0.0019 \\
0.0011\end{array}$ & & 1. $4 E-07$ \\
\hline Pu $1.22 a$ & $\mathrm{up}_{\mathrm{p}}$ & 500 & No & 7.4 & 4440 & & -0.0002 & $-4.5 E-08$ \\
\hline $\mathrm{Pu} 123$ & Down & 500 & No & 7.4 & 4440 & 0.0008 & 0.0005 & $1.1 \mathrm{E}-07$ \\
\hline $\mathrm{Pu} \quad 123 \mathrm{a}$ & Down & 500 & No & 7.4 & 4440 & 0.0060 & 0.0047 & 1. $1 E-06$ \\
\hline Pu 124 & $U_{p}$ & 1250 & Yes & 30.3 & 18180 & 30.06 & 30.06 & $1.7 E-03$ \\
\hline Pu 124a & $v_{p}$ & 1250 & Yes & 32.9 & 1.9740 & 2.924 & 2.923 & $1.5 E-04$ \\
\hline Pu 125 & Down & 1250 & Yes & 33.7 & 20220 & 0.0425 & 0.0412 & 2. $0 E-06$ \\
\hline $\mathrm{Pu} 125 \mathrm{a}$ & Down & 1250 & Yes & 28.9 & $\begin{array}{l}20220 \\
17340\end{array}$ & 0.0336 & 0.0323 & $1.9 E-06$ \\
\hline Pu 126 & Up & 1250 & No & 35.9 & $\begin{array}{l}1 / 340 \\
21540\end{array}$ & 0.0413 & 0.0400 & $1.9 E-06$ \\
\hline $\mathrm{Pu} 126 \mathrm{a}$ & Up & 1250 & No & 36.3 & $\begin{array}{l}21540 \\
21780\end{array}$ & 0.0275 & 0.0262 & 1. $2 E-06$ \\
\hline $\mathrm{Pu} 127$ & Down & 1250 & No & 31.8 & 19080 & 0.0697 & 0.0684 & $3.6 \mathrm{E}-06$ \\
\hline Pu $127 a$ & Down & 1250 & No & 32.2 & 19320 & 2.567 & 2.566 & 1. $3 E-04$ \\
\hline
\end{tabular}

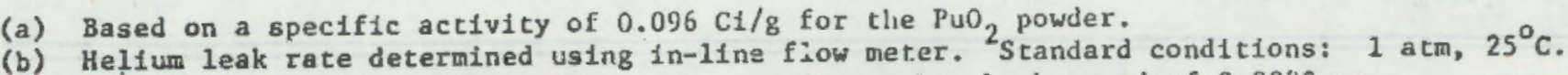

(c) The net total is the amount above the containment box background of $0.0030 \mu \mathrm{g}$.

(d) The net total is the amount above the average containment box background of 0.0013 lig.

(e) Best estimate of total flow in standard $\mathrm{cm}^{3}$. Standard conditions: 1 atm, $25^{\circ} \mathrm{C}$. 
TABLE B15. SUMMARY OF PuO 2 LEAK RATE EXPERIMENTS USING A 50-um ORIFICE(a)

\begin{tabular}{|c|c|c|c|c|c|c|c|c|}
\hline \multirow[b]{2}{*}{$\begin{array}{l}\text { Run } \\
\text { Number }\end{array}$} & \multirow[b]{2}{*}{$\begin{array}{c}\text { Tube } \\
\text { Position }\end{array}$} & \multirow{2}{*}{$\begin{array}{c}\text { Hellum } \\
\text { Pressure, } \\
\text { pat }\end{array}$} & \multirow[b]{2}{*}{ Vibration } & \multirow{2}{*}{$\begin{array}{l}\text { Helium } \\
\text { Leak } \\
\text { Rate } \\
\text { scc } / \text { sec }\end{array}$} & \multirow{2}{*}{$\begin{array}{l}\text { Total } \\
\text { Hellum } \\
\text { Flow } \\
\text { scc (e) }\end{array}$} & \multicolumn{2}{|c|}{$\begin{array}{l}\text { Quantity of } \mathrm{PuO}_{2} \\
\text { Detected, } \mathrm{ug}^{\mathrm{i}(\mathrm{b}}\end{array}$} & \multirow{2}{*}{$\begin{array}{c}\text { Mass Flow } \\
\text { Ratio } \\
\mu g / c c\end{array}$} \\
\hline & & & & & & Total & $\operatorname{Total}_{\text {Net }}^{\text {(d) }}$ & \\
\hline Pu 128 & Up & 1000 & Yes & $r$ & 775 & 12.45 & 12.45 & 1. $6 \mathrm{E}-02$ \\
\hline Pu 129 & Up & 1000 & Yes & 9.6 & 34560 & 0.2905 & 0.2840 & 8. $2 E-06$ \\
\hline Pu $128 \mathrm{a}$. & Up & 1000 & Yes & - & 775 & 0.0287 & 0.0284 & 3. $7 E-05$ \\
\hline Pu 129a & Up & 1000 & Yes & 13.4 & 48240 & 0.1463 & 0.1398 & $2.9 E-06$ \\
\hline $\mathrm{Pu} 128 \mathrm{~b}$ & Up & 1000 & Yes & - & 775 & 0.0132 & 0.0129 & 1. $7 \mathrm{E}-05$ \\
\hline Pu $129 b$ & $U_{p}$ & 1000 & Yes & 13.0 & 46800 & 0.0196 & 0.0131 & $2.8 E-07$ \\
\hline $\mathrm{Pu} 128 \mathrm{c}$ & Up & 1000 & Yes & $\overline{-}$ & 775 & 0.1622 & 0.1619 & $2.1 E \sim 04$ \\
\hline Pu $129 c$ & $U_{p}$ & 1000 & Yes & 13.7 & 49320 & 0.0997 & 0.0932 & $1.9 E-06$ \\
\hline Pu $128 d$ & $U_{p}$ & 1000 & Yeg & - & 775 & 0.0063 & 0.0060 & $7.7 \mathrm{E}-06$ \\
\hline $\mathrm{Pu} 129 \mathrm{~d}$ & $U_{p}$ & 1000 & Yes & 14.8 & 53280 & 0.0600 & 0.0535 & $1.0 \mathrm{E}-06$ \\
\hline Pu 131 & Up & 1000 & No & 14.5 & 8700 & 0.0562 & 0.0549 & $6.3 E-06$ \\
\hline Pu 131a & Up & 1000 & No & 13.7 & 8220 & 0.1111 & 0.1098 & 1. $3 E-05$ \\
\hline Pu 131b & $U_{p}$ & 1000 & No & 21.2 & 12720 & 0.0149 & 0.0136 & $1.1 \mathrm{E}-06$ \\
\hline Pu 131c & Up & 1000 & No & 16.3 & 9780 & 0.0105 & 0.0092 & $9.4 \mathrm{E}-07$ \\
\hline Pu 131d & $U_{p}$ & 1000 & No & 14.1 & 8460 & 0.0046 & 0.0034 & $4.0 E-07$ \\
\hline Pu 131e & Up & 1000 & No & 13.7 & 8220 & 0.0046 & 0.0033 & $4.0 \mathrm{E}-07$ \\
\hline Pu $131 f$ & $u_{p}$ & 1000 & No & 13.0 & 7800 & 0.0026 & 0.0013 & $1.7 \mathrm{E}-07$ \\
\hline Pu 1318 & Up & 1000 & No & 11.5 & 6900 & 0.0050 & 0.0037 & $5.4 \mathrm{E}-07$ \\
\hline Pu $131 \mathrm{~h}$ & Up. & 1000 & No & 13.4 & 8040 & 0.0040 & 0.0027 & $3.4 E-07$ \\
\hline $\mathrm{Pu} 1311$ & Up & 1000 & No & 12.6 & 7560 & 0.0342 & 0.0329 & 4. $4 E-06$ \\
\hline Pu 131J & $U_{p}$ & 1000 & No & 13.4 & 8040 & 0.0042 & 0.0029 & $3.6 \mathrm{E}-07$ \\
\hline Pu $131 k$ & Up & 1000 & No & 12.6 & 7560 & 0.0066 & 0.0053 & $7.0 \mathrm{E}-07$ \\
\hline Pu 1311 & Up & 1000 & No & 21.2 & 12720 & 0.0131 & 0.0118 & $9.3 \mathrm{E}-07$ \\
\hline Pu 131n & Up & 1000 & No & 20.1 & 12060 & 0.0112 & 0.0099 & 8. $2 \mathrm{E}-07$ \\
\hline Pu $131 n$ & Up & 1000 & No & 19.7 & 11820 & 0.0038 & 0.0025 & $2.1 E-07$ \\
\hline
\end{tabular}

\footnotetext{
(a) Based on a specific activity of $0.096 \mathrm{Cl} / 8$ for the $\mathrm{PuO}_{2}$ powder.

(b) Helium leak rate determined using in-11ne flow meter. ${ }^{2}$ Standard conditions: 1 atm, $25^{\circ} \mathrm{C}$.

(c) The net total is the amount above the containment box background of $0.0030 \mathrm{\mu g}$.

(d) The net total is the amount above the average contatinment box background of $0.0013 \mu g$.

(e) Best esifmate of total flow in standard $\mathrm{cm}^{3}$. Standard conditions: $1 \mathrm{~atm}, 25^{\circ} \mathrm{C}$.
} 

NUREG/CR-1302

PNL-3278

RT

\section{DISTRIBUTION}

No. of

Copies

OFFSITE

A. A. Churm

DOE Patent Division

9800 S. Cass Avenue

Argonne, IL 60439

J. J. Davis

Office of Nuclear Regulatory

Research

Division of Safeguards, Fuel Cycle and Environmental Research

NRC Division of Research

Washington, DC 20555

10 W. Lahs

Office of Nuclear Regulatory

Research

Division of Safeguards, Fue 1 Cycle and Environmental Research

NRC Division of Research

Washington, DC 20555

Frank Swanberg, Jr.

Office of Nuclear Regulatory

Research

Division of Safeguards, Fuel Cycle and Environmental Research

NRC Division of Research

Washington, DC 20555

Char les E. MacDona1d

NRC Office of Nuclear Material

Safety \& Safeguards

Transportation Branch

Washington, DC 20555

C. Ross Chappe11

NRC Office of Nuclear Material

Safety \& Safeguards

Transportation Branch

Washington, DC 20555
No. of

Copies

William H. Lake

NRC Office of Nuclear Material

Safety \& Safeguards

Transportation Branch

Washịngton, DC 20555

261 NRC Division of Technical

Information and Document Control

Washington, DC 20555

Donald R. Hopkina

NRC Division of Standards

Washington, DC 20555

2 DOE Technical Information Center

U.S. Department of Energy

P.0. Box 62

Oak Ridge, TN 37830

John A. Andersen

Sandia Laboratories

Albuquerque, NM 87115

J. K. Cole

Sandia Laboratories

Albuquerque, NM 87115

M. Pobereskin

Battelle Memorial Institute 505 King Avenue

Columbus, $\mathrm{OH} 43201$

25 W. J. Madia

Battelle Memorial Institute

505 King Avenue

Columbus, $\mathrm{OH} 43201$

E. W. Schmidt

Battelle Memorial Institute

505 King Avenue

Columbus, OH 43201 
No of

Copies

J. D. Yesso

Battelle Memorial Institute

505 King Avenue

Columbus, $\mathrm{OH} 43201$

ONSITE

50 Pacific Northwest Laboratory

T. J. Bander

C. E. Elderkin

R. K. Hadlock

J. W. Johnston

J. Mishima (35)

P. C. Owzarski

L. C. Schwendiman

S. L. Sutter

R. K. Woodruff

Publishing Coordination (2)

Technical Information (5) 


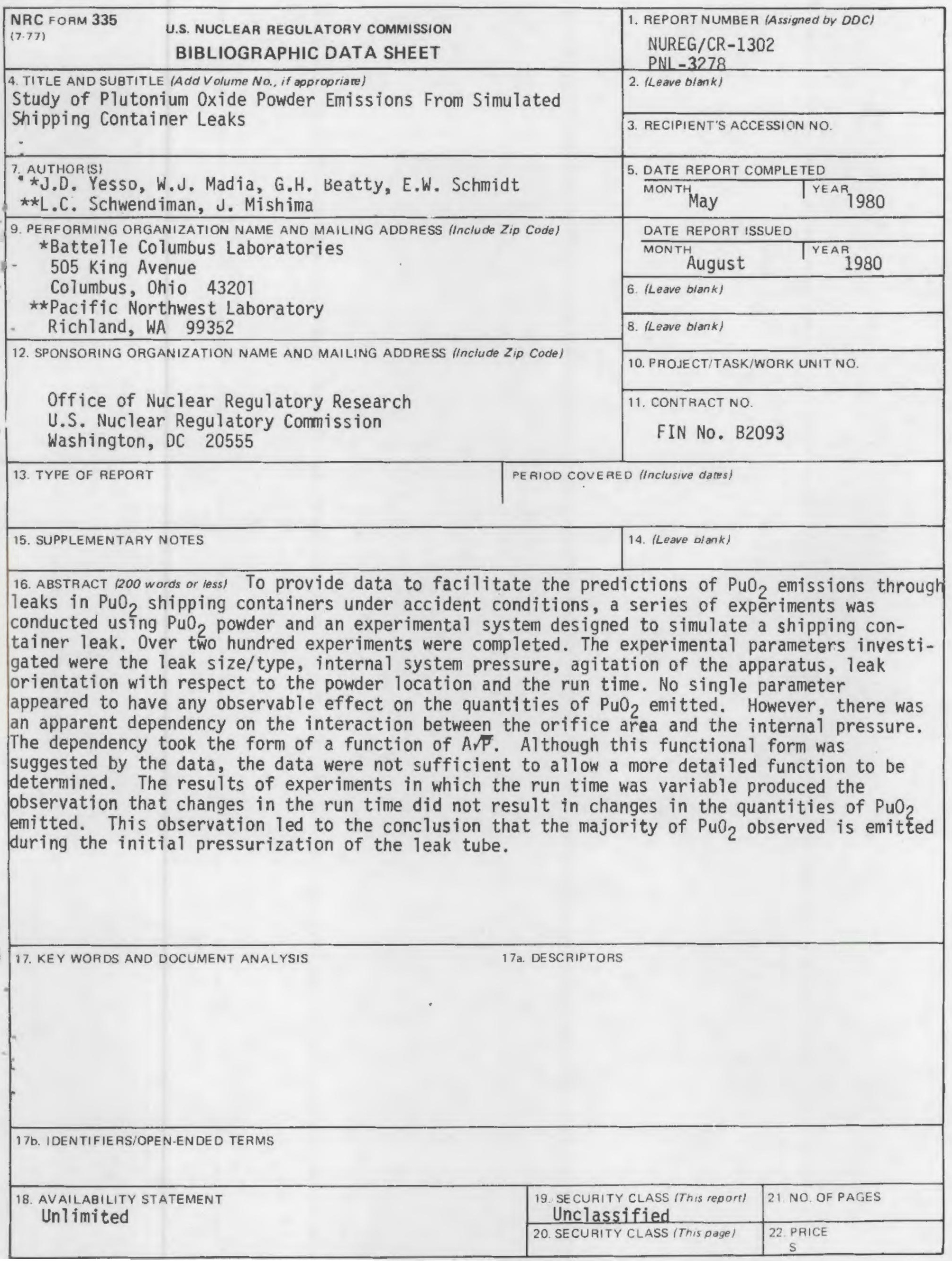




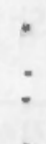

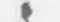

\%

$-1$

+

+

+

+

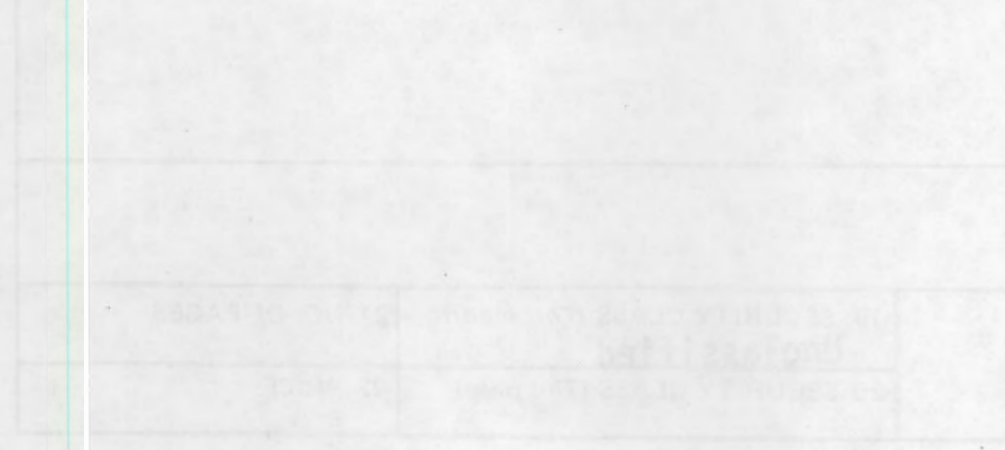

